\title{
Any Light Particle Search II Technical Design Report
}

\author{
Robin Bähre $^{a}$, Babette Döbrich ${ }^{b}$, Jan Dreyling-Eschweiler ${ }^{b}$, Samvel Ghazaryan ${ }^{b}$, \\ Reza Hodajerdi $^{b}$, Dieter Horns ${ }^{c}$, Friederike Januschek ${ }^{b}$, Ernst-Axel Knabbe ${ }^{b}$, Axel \\ Lindner ${ }^{b}$, Dieter Notz $^{b}$, Andreas Ringwald ${ }^{b}$, Jan Eike von Seggern ${ }^{b}$, Richard \\ Stromhagen $^{b}$, Dieter Trines $^{b}$, Benno Willke ${ }^{a}$ \\ ${ }^{a}$ Albert Einstein Institute, \\ Callinstr. 38, 30167 Hannover, Germany \\ ${ }^{b}$ Deutsches Elektronen-Synchrotron (DESY), \\ Notkestr. 85, 22607 Hamburg, Germany \\ ${ }^{c}$ University of Hamburg, \\ Institut für Experimentalphysik, Luruper Chaussee 149, 22761 Hamburg, Germany \\ E-mail: axel.lindnerdesy.de
}

\begin{abstract}
This document constitutes an excerpt of the Technical Design Report for the second stage of the "Any Light Particle Search" (ALPS-II) at DESY as submitted to the DESY PRC in August 2012 and reviewed in November 2012. ALPS-II is a "Light Shining through a Wall" experiment which searches for photon oscillations into weakly interacting sub-eV particles. These are often predicted by extensions of the Standard Model and motivated by astrophysical phenomena. The first phases of the ALPS-II project were approved by the DESY management on February 21st, 2013.
\end{abstract}

KEYWORDS: Dark Matter detectors (WIMPs, axions, etc.), Large detector systems for particle and astroparticle physics; Resonant Detectors.

${ }^{*}$ Corresponding author. 


\section{Contents}

1. Introduction 2

2. Motivation and science goals for ALPS-II

2.1 Fundamental physics with low-mass, weakly coupled particles

2.1.1 The hunt for WISPs and their connection to fundamental physics questions

2.1.2 The case for axions and axion-like particles 6

2.1.3 Motivation for hidden photons and minicharged particles 9

2.1.4 Further scientific impact of ALPS-II 10

2.2 Discovery potential at the three stages of ALPS-II 10

2.2.1 Experimental aims in the search for ALPs at ALPS-IIc 10

2.2.2 Discovery potential for HPs and MCPs at ALPS-II 13

3. The experiment 17

3.1 Overview 17

3.1.1 Stages of the experiment 117

3.1.2 ALPS-II operation modes and signal interpretation 19

3.2 Laser and optics 22

3.2.1 Technical challenges 22

3.2.2 Conceptual design 22

3.2 .3 Expected performance 28

3.3 Cleanroom design 29

3.3.1 General considerations 29

3.3 .2 The cleanrooms 30

3.4 Detection system 32

3.4.1 Technical challenges 32

3.4.2 Conceptual design 33

3.4.3 Expected performance 37

3.5 Magnets and cryogenics 41

3.5.1 Introduction 41

3.5.2 Straight HERA dipoles 43

3.5.3 The ALPS-IIc magnet string 48

3.5 .4 Alignment 49

3.6 Vacuum system 54

3.6.1 The vacuum system for the optical resonators of ALPS-IIb

3.6.2 The vacuum system for the optical resonators of ALPS-IIc 54

3.6 .3 The insulation vacuum system

3.6.4 Increasing the refractive index in the optical resonators of ALPS-IIc 56

3.7 Data acquisition 59

3.8 Site considerations for ALPS-IIb and IIc 62 


\section{Introduction}

Understanding the fundamental constituents and forces of nature is one of the strongest motivations for society to support experimental and theoretical physics. This has been again proven for example by the enormous public resonance on the likely discovery of the Higgs boson at the LHC and shows up continuously in the interest in astronomical and cosmological questions. Indeed, the progress is impressive. Mainly accelerator based research has led to the development of the so-called Standard Model of particle physics, which is able to describe experimental results up to \%o-level accuracy. However, while such experiments have been extremely successful to fill the gaps in the Standard Model as predicted a few decades ago, evidence for physics beyond this theoretical framework has mounted from astrophysical and cosmological observations. Most prominent are Dark Energy and Dark Matter. So far accelerator based experiments at the energy or intensity frontiers have not been able to identify explanations towards understanding these phenomena.

Hence it appears to be natural to ask whether physics beyond the Standard Model may hide at another frontier. And in fact such a possible frontier is known since the early 1980ties, when the apparent CP-conservation in QCD was traced back to a hypothetical new particle, the axion. It was quickly shown that the axion, if it exists, should be extremely light and weakly interacting to escape experimental bounds. Such an axion was named "invisible". It poses a new "low-energy frontier" to the Standard Model hinting at uncharted territory beyond the reach of accelerator based experiments. In recent years it became clear that the axion might be just one example of many different "weakly interacting slim particles" (WISPs) as we will describe in Sect. 2.

Experiments searching for WISPs usually rely on photon-WISP interactions. As will be shown in the subsequent chapter, one can distinguish between WISPs with the same quantum numbers as photons (allowing for kinetic mixing with photons), and pseudo-scalar or scalar bosons which require two-photon interactions. The proposed second stage of the ALPS experiment will be sensitive to both kinds of WISPs and even other predicted WISPy particles.

Typically in such an experiment one of the two photons is provided by a laser beam, while the other is supplied by a strong magnetic field. In such environments the interactions between WISPs and photons take place in a coherent fashion. The interaction probability is many orders of magnitude larger than in accelerator based beam dump experiments for example. A virtual or real production of WISPs could give rise to spectacular experimental observations ranging from polarization effects to the sudden appearance of light in a seemingly perfectly shielded environment. The ALPS-II proposal concentrates on the latter idea by trying to shine light through a wall. 


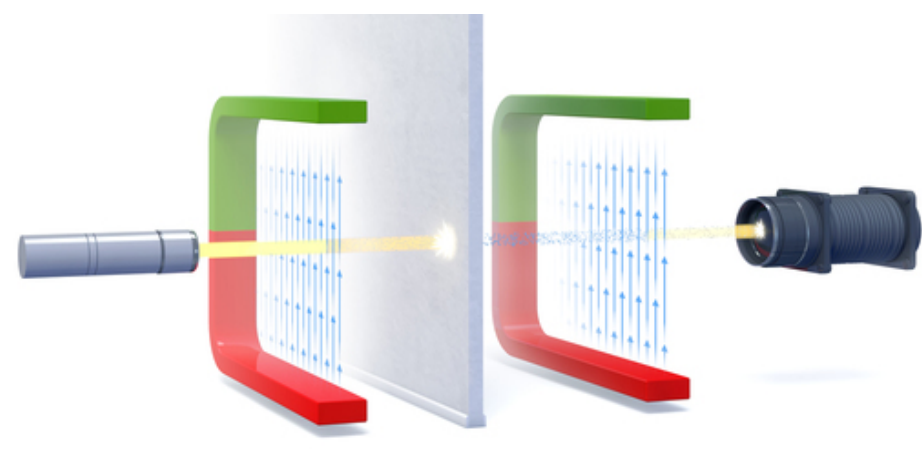

Figure 1. The principle of a light-shining-through a wall experiment: light, typically from a strong laser, is shone into a magnetic field. WISPs could be created by interaction of the laser light with the magnetic field or just by kinetic mixing. A barrier blocks the light, but WISPs easily traverse it. In the second part of the experiment behind the wall, some WISPs convert back to photons giving rise to the impression of light shining through a wall. The WISPs have to move over macroscopic distances, so that only the production of on-shell particles is relevant here.

A clear observation of light generated in a very well shielded environment could only be explained by yet unknown WISPs which pass any barrier. The principle of "light-shining-througha-wall" (LSW) experiments is shown in Figure 1 .

WISPs produced by laser light as well as reconverted photons originating from these WISPs have laser-like properties. This allows to

- guide them through long and narrow tubes inside accelerator dipole magnets and

- to exploit resonance effects by setting up optical resonators (cavities).

The ALPS-I experiment at DESY has improved previous results by a factor of 10 in 2010. Future increases in sensitivity rely basically on three corner stones:

- An optical resonator in front of the wall is the best means to "store" the injected laser light and thus to amplify the available laser light power. The resonator houses a standing light wave created by the superposition of the light waves reflected by the two mirrors defining the resonator. In the resonator the field strength of the light wave moving towards the "wall" is much larger than the field strength of the injected laser light. The ratio of the square of both field strengths is called the power built-up factor in the following.

- With a second resonator behind the wall the back conversion probability of WISPs into photons can be greatly enhanced. To understand this, one has to keep in mind that an optical resonator also works for very faint light in exactly the same way as sketched above. Indeed it has been verified experimentally in the microwave regime that even a light wave with an expectation value for the number of photons inside the resonator much below one is amplified as anticipated. Now the wave function of the WISPs propagating in the resonator behind the wall can be described as consisting out of a large "sterile" part and a tiny photonic component. Consequently, the photonic component is amplified in the resonator in exactly 
the same fashion as "ordinary" light and the conversion probability into photons increased accordingly. However, this approach requires that the resonators in front and behind the wall are tuned to the same frequency and spatial mode (as the laser photons and the photons from regenerated WISPs have exactly the same properties) and have a negligible relative phase jitter only (so that the WISP beam passing the wall can inject photons into the second resonator in the same manner as laser light is injected into a resonator).

- The magnetic length is increased by using a string of dipole magnets. As the production of WISPs and the regeneration of photons takes place in a coherent fashion, a 20-fold increase in the magnetic length would result in the same increase in the sensitivity to the WISP-photon coupling strength.

The ALPS-II proposal describes how to combine existing infrastructure at DESY (HERA dipole magnets, long straight sections in the HERA tunnel, cryogenics) with world-leading expertise in laser technology (derived from experience at gravitational wave interferometers) and new optical detectors (superconducting single-photon counters) to achieve sensitivities in WISP searches more than three orders of magnitude better than at existing laboratory experiments.

The interest in the low-energy frontier has changed in the last five to ten years. This shows up in the growing participation in the PATRAS workshop series (see http://axion-wimp. desy. de), recent workshops in the US and the document submitted by international authors for the discussion of the update of the European strategy for particle physics (http://indico. cern.ch/contributionDisplay . py?contribId=105\\&confId=175067). Cornerstones were the CAST helioscope at CERN, which searches for axion emission from the sun compatible with existing solar models, new theoretical insights into extensions of the Standard Model, which very often predict numerous new particles at the low-energy frontier, and recently observed astrophysical puzzles which could be explained by such new particles. Consequently new experimental activities started world-wide. Very often they made use of the infrastructure of large accelerator laboratories. One example was the first stage of the ALPS ("Any Light Particle Search") experiment at DESY. It utilized a HERA dipole magnet combined with a strong cw laser driving an optical resonator inside the magnet. By combining the know-how of particle physicists and experts of gravitational wave interferometers, ALPS-I reached the world-wide best sensitivity on the photon coupling for new weakly-interacting, low mass particles amongst laboratory searches.

ALPS-I as well as competing experiments at CERN, FNAL, JLAB and elsewhere can be described as quick "shots into the dark". They aimed for fast returns using existing equipments (in the ALPS-I case a HERA dipole magnet accidentally still installed at a test bench) given strong budget constraints. None of these experiments have shown evidence for physics beyond the Standard Model. However, the realizability of such experiments based on new collaborations of different branches of physics has been proven successfully in these prototype approaches. A second generation ALPS-II experiment promises to increase the experimental sensitivity in axion-like particle searches by more than three orders of magnitude (!). It will allow to access the parameter space of new hypothetical particles predicted by the abovementioned astrophysical phenomena and predictions of low-energy incarnations of string theory in the laboratory. It will surpass the strongest constraints on axion-like particles provided presently by CAST in the low-mass range whilst offering full laboratory control 
over all experimental parameters. ALPS-II will use existing HERA dipoles to be installed in a straight section of the HERA tunnel. This will be achieved at comparably modest costs while the impact of any discovery could hardly be overestimated. However, there are competing activities mainly in the US, which pose time demands on the realization of ALPS-II. For these reasons, a number of theorists and experimentalists have proposed in the recent submission to the European Strategy Group to realize ALPS-II.

\section{Motivation and science goals for ALPS-II}

In the following, we first outline in detail the motivation arising from theory and astrophysics for the search for new physics at the low-energy frontier, and give a first sketch of the potential impact of ALPS-II in this field, see Sect. 2.1. Secondly, we detail on the prospected sensitivity of ALPS-II derived from the experimental parameters, cf. Sect. 2.2. Details on the experiment itself will follow in Sect. [3.

\subsection{Fundamental physics with low-mass, weakly coupled particles}

\subsubsection{The hunt for WISPs and their connection to fundamental physics questions}

Whilst at the moment the LHC strongly supports our current view of fundamental particle interactions, the Standard Model, it is disturbing that our standard cosmological model still requires at least two more components whose nature is at the moment completely unknown: these are Dark Matter (DM) and Dark Energy. In addition, recent cosmological data suggests even a third component, Dark Radiation, a weakly interacting relativistic particle contributing to the cosmological radiation density like a neutrino. Besides the missing explanation for this "dark sector", a second shortcoming of the Standard Model is its omittance of a quantization of gravity. A worthwhile and enormous effort is being made to gain an understanding of these tantalizing mysteries of nature, most prominently at the high energy frontier. However, only a comprehensive search for physics beyond the Standard Model can make sure that we do not overlook the answers to the questions we have to nature.

In particular, new particles may have eluded our experimental searches so far not only if they are very massive, but also easily if they are rather light but weakly coupled [1], 2].

For this reason, in this document, we propose an unprecedented search for weakly-interacting slim particles (WISPs) in a LSW setup, using high laser intensities as a lever arm. LSW makes use of the fact that WISPs coupled to photons can be produced inside an optical resonator ("generation region"). Subsequently WISPs can traverse a barrier ("wall") which is opaque to photons due to the WISPs' feeble interaction with the barrier. Thereupon some WISPs can be reconverted into photons behind the barrier ("regeneration region"). Thus, via WISPs, light could seemingly "shine through a wall"'.

We herein build on the pioneering experience with the ALPS-I experiment [ remains the currently most sensitive LSW-setup [8] worldwide, see [6]. Other optical LSW setups

\footnotetext{
${ }^{1}$ Note that the "Standard Model background for LSW" due to neutrinos or gravitons is negligible for the proposed

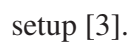


included LIPSS [9, [10] at Jefferson Lab, GammeV [11, 12] at Fermilab, OSQAR [13, 14] at CERN as well as a LSW setups with BMV [15, [16] and BFRT [17] (see also Tab. (4) for the respective magnetic lengths). Note that for OSQAR, the evaluation of data is not yet completed [18]. Also, a proposal for a consecutive experiment at Fermilab, "REAPR" has been made [19].

In a nutshell, the enhanced sensitivity of ALPS-II (three orders of magnitude in comparison to ALPS-I) will be due to the increased magnetic length, an optical resonator on the regeneration side and the introduction of advanced detector technology.

As argued below, the proposed ALPS-II experiment might thus contribute to our answering the above mentioned important fundamental physics questions. ALPS-II will be sensitive to uncharted parameter ranges of several WISPs, in particular to "axion-like particles" (ALPs), hidden photons (HPs), minicharged particles (MCPs) and certain scalar fields of massive gravity theories. The theory case for these particles and the corresponding ALPS-II sensitivity is outlined below.

Pertaining to experiments which exploit the WISPs' coupling to photons, three major search strategies are implemented today [20]: Firstly, so-called Haloscopes work under the assumption that axions, ALPs or HPs constitute a large fraction of the Dark Matter halo also present in our solar system. If this holds true, a sizable number of Dark Matter axions, ALPs or HPs are present within state-of-the-art haloscope detectors, making WISPs detectable through a WISP-photon conversion process. Secondly, so-called Helioscopes make use of the WISP particle flux which should reach us from the sun. The most prominent example for such a setup is the "Cern Axion Solar Telescope" (CAST). Thirdly, light-shining-through-walls setups, as also proposed in this document, have become an indispensable tool in the search for WISPs. Although they are less sensitive than Haloscopes and Helioscopes at first sight, they have at least two different advantages which make them indispensable: They are particularly flexible in the sense that they maintain full access and control over the WISP production and regeneration regions. For example, controlling and altering the laser polarization as well as the magnetic field strength often provides access to more particles species. Also, control over the production side eventually enables the locking of a second optical cavity on the regeneration side. This will eventually make the proposed LSW setup ALPSII more sensitive than today's best existing helioscopes in the lower mass region. In addition, note that LSW setups are sensitive whether or not WISPs are only a fraction of Dark Matter or no Dark Matter at all.

\subsubsection{The case for axions and axion-like particles}

Besides the Higgs particle, the most established proposal for a fundamental spin- 0 particle in nature is the axion [21, 22]. As a consequence of the spontaneous breakdown of the so-called PecceiQuinn (PQ) [23] symmetry, it provides the most viable solution to the strong CP problem. In a nutshell, evidence of the axion particle would explain why the observed CP-violation in the sector of strong interactions is off by at least 10 orders of magnitude from expectation. The mechanism which gives rise to the axion can be generalized to generic (pseudo-) scalars $a$ coupled weakly to two-photons $\sim g_{a \gamma}$, so-called axion-like particles (ALPs). For axions, the relation between their mass and coupling is given through the color anomaly of the PQ symmetry, cf. the yellow band in Fig. 2. Thus, intrinsically, for typical axion models this mass-coupling relation is such that only a small part of its parameter space could be searched yet. 


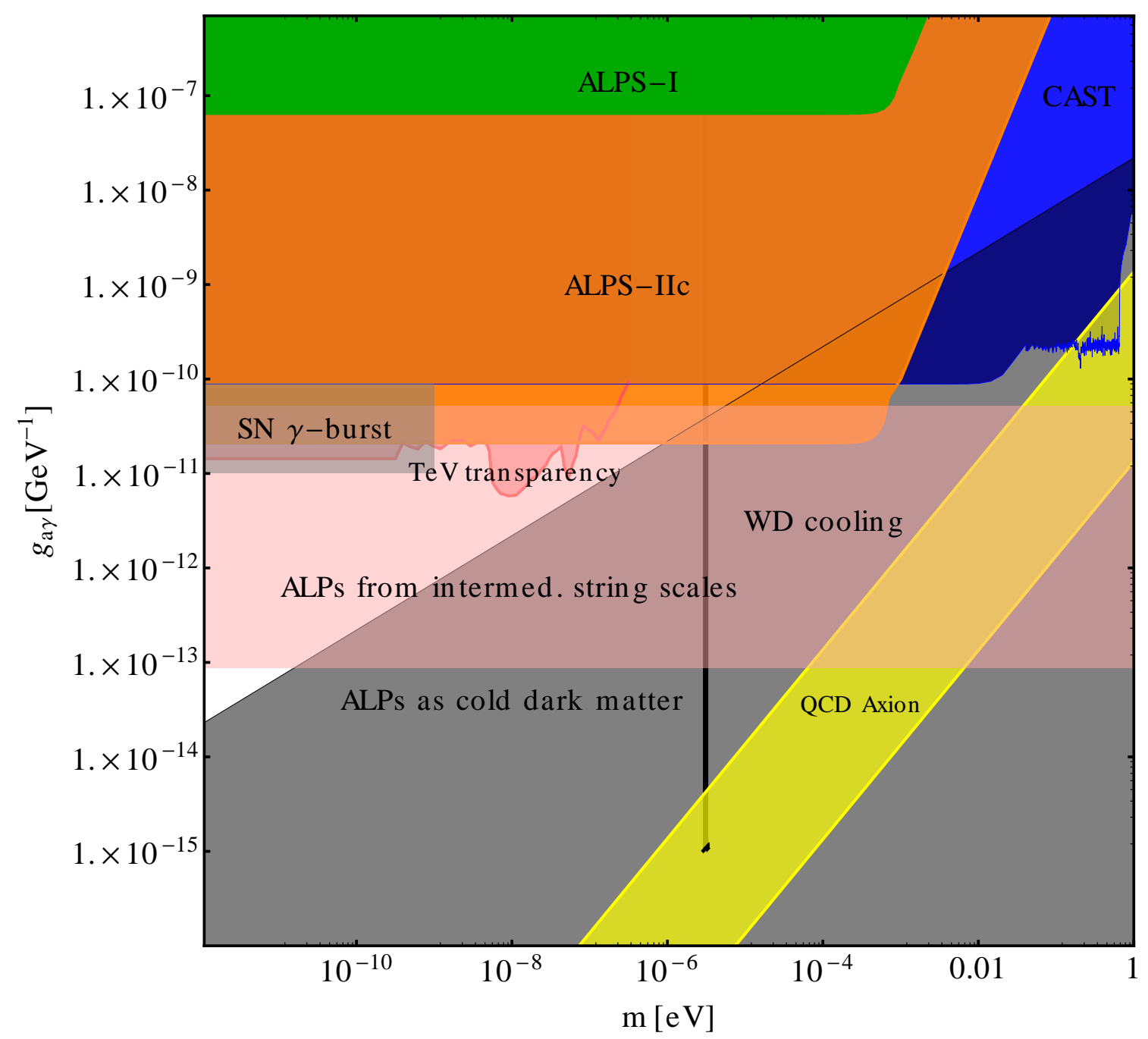

Figure 2. Schematic overview of the sensitivity reach of the final stage of ALPS-II, ALPS-IIc depicting its scientific impact (The detailed sensitivity range of ALPS-IIc is given in Fig. 3.). As a yellow band, generic QCD axion models are indicated. For comparison, the ALPS-I results (in green, max. sensitivity $g_{a \gamma} \gtrsim 7 \times 10^{-8} \mathrm{GeV}^{-1}$ ) and the currently most stringent bounds on ALPs in this mass region from the CAST helioscope [24] (in blue) are shown. As visible, ALPS-IIc (in orange, max. sensitivity $g_{a \gamma} \gtrsim 2 \times 10^{-11} \mathrm{GeV}^{-1}$ ) will surpass the CAST bounds in the lower mass region and tackle parameter regions in which ALPs are motivated by fundamental particle physics questions (cold Dark Matter candidates, in gray, and predictions from string theory around $g_{a \gamma} \sim 10^{-12} \mathrm{GeV}^{-1}$, not colored) as well as astrophysical hints: TeV transparency (red, fiducial region, cf. [25]) and WD cooling (light red band)). In addition the parameter region excluded from the 1987a super-nova burst is indicated. The predictions from theory and astrophysical considerations are generally certain within about an order of magnitude. Note that the parameter range between $\mu \mathrm{eV} \lesssim m \lesssim 1 \mathrm{meV}$ is particularly interesting in the context of axion Dark Matter, and currently most successfully probed by ADMX [26] (cf. black line). ALPS, on the other hand, can be Dark Matter in a much wider mass range. For a recent comprehensive overview of the ALPs parameter space, see [27, 28]. 
Generic ALPs, however, do not fall under the mass-coupling relation of axions, and thus can be realized in a much wider parameter space, see Fig. 目. Like the axion, ALPs can be realized as pseudo-Nambu-Goldstone bosons of symmetries broken at very high energies. Very strong motivation for their existence indeed comes from string theory which tries to tackle the Standard Model's shortcoming of omitting gravity. Generically in these theories, a rich spectrum of light (pseudo-)scalars with weak couplings arises. Of particular relevance to the physics case of ALPS-II are intermediate string scales $M_{\mathrm{s}} \sim 10^{9}$ to $10^{12} \mathrm{GeV}$, which can contribute to the natural explanation of several hierarchy problems in the SM. In these theories, ALPs generically exhibit a coupling to photons which lies in a parameter range that is accessible to the final stage of the ALPS-II experiment, ALPS-IIc: The ALP coupling to two-photons is $g_{a \gamma} \sim \frac{\alpha}{2 \pi} \frac{c}{f_{a}}$, where $f_{a}$ is the symmetry breaking scale, $\alpha$ the fine structure constant, $c$ is a constant of $\mathscr{O}(1)$ and $f_{a} \sim M_{\mathrm{s}}$. Thus ALPs from intermediate string scales can very well occur in the $g_{a \gamma} \sim 10^{-12} \mathrm{GeV}^{-1}$ regime [29], as sketched in Fig. 2. Unlike for axions, for axion-like relation the ALP-photon coupling to couplings to other particle species is not straightforward ${ }^{2}$. It is important to follow therefore complementary search strategies. While ALPS-II is only sensitive to the ALP coupling to photons, other experiments and astrophysical arguments probe also (pseudo-) scalar couplings, e.g., to nucleons [30] or neutrons [31].

However, axions and ALPs can not only be a low-energy window to new physics in the ultraviolet, but they are also well-motivated Dark Matter candidates. Generally, one of the most prominent candidates for Dark Matter are "weakly interacting massive particles" (WIMPs) whose drawing power is twofold: WIMPs at the weak scale provide the right relic abundance and new particles at these scales are favored in particular by supersymmetry. A lot of effort has thus be put into detecting WIMPs as Dark Matter directly as well as indirectly at, e.g., the LHC. However, although no final conclusive statements can yet be made: The fact that the LHC as well as the currently most sensitive direct search Xenon100 have found no such particle, posing heavy restrictions on some popular models [32], should remind us that other well-motivated Dark Matter candidates do exist. In particular, axions and other WISPs constitute viable Dark Matter candidates.

For axions, the Dark Matter option has been mainly explored since the 80s particularly by the "axion dark matter eXperiment" (ADMX). With their ongoing effort and the contribution of setups with complementary mass range [33-35], well-established methods are available to explore the cold Dark Matter axion window. For other WISPs, the viable Dark Matter parameter space is largely unexplored as recently demonstrated [28]. Note that if produced non-thermally through the misalignment mechanism, even very light WISPs can be cold Dark Matter. Again, it is noteworthy that ALPS-II can explore in part ALP Dark Matter options as indicated in Fig. Z and might therefore complement upcoming experiments dedicated to WISPy Dark Matter detection.

In addition to the motivations for ALPs that arise from UV-completions of the Standard Model and their possible connection to Dark Matter, two hints from astro-particle physics strengthen the physics case for searching axion-like particles ALPS-II even further.

The first hint pertains to the propagation of cosmic gamma rays with energies above $\mathscr{O}(100) \mathrm{GeV}$. Even if no absorbing matter blocks the way of these high energy photons, absorption must be ex-

\footnotetext{
${ }^{2}$ While it is true that for Goldstone bosons the coupling to the respective particle types $x$ will obey $g_{a x} \sim C_{a x} / f$, with $f$ the symmetry breaking scale, however, the coefficient $C_{a x}$ is model dependent and could even be 0 for some $x$.

${ }^{3}$ Note that renormalization group studies formally justify a comparison of ALPs constraints arising from different
} 
pected as the gamma rays deplete through electron-positron pair production through interaction with extragalactic background light. However, the observed energy spectra do not seem to match the absorption feature inferred from this argument [38]. Axion-like particles could provide a resolution to this puzzle. Here, the anomalous transparency can be explained if photons convert into ALPs in astrophysical magnetic fields. The ALPs then travel unhindered due to their weak coupling to normal matter. Close to the solar neighborhood, ALPs could then be reconverted to high-energy photons. A sizable number of authors is intensely studying this resolution to the transparency problem, see, e.g., [39-41] to name only a few. Typically, ALPs related to the transparency hint are predicted to lie in the $g_{a \gamma} \sim 10^{-11}$ to $10^{-12} \mathrm{GeV}^{-1}$ coupling-region. In Fig. 2 it can be seen that ALPS-II can explore a sizable parameter space related to this hint. Note that the favored parameter regions related to this hint can be covered with ALPS-II almost entirely [25].

The second hint pertains to the longstanding puzzle of the anomalously large cooling rate of white dwarfs (WD) which receives additional support by the most recent studies of the WD luminosity function [42] and in some cases to the observed decrease in pulsation period of these objects [43]. The cooling excess can be attributed to axions with $f_{a} \sim 10^{8}$ to $10^{9} \mathrm{GeV}$ if they couple to electrons. In a generic case the axion (or ALP) will also have a photon coupling. In Fig. Z the corresponding target region ${ }^{4}$ is depicted, labeled "WD cooling".

A number of additional astrophysical observations not indicated in Fig. 目 have been considered to search for signatures of ALPs or - in the absence of a signal - constrain the parameters. Observations of linearly polarized emission from magnetized WDs [44] and changes of the linear polarization from radio galaxies (e.g., [45]) provides complementary approaches to search for signatures of ALPs. The current limits are close to $g_{a \gamma} \sim 10^{-11} \mathrm{GeV}^{-1}$ albeit with uncertainties related to the underlying assumptions.

Note that of course upcoming data from Cherenkov or space-bound telescopes might very well further strengthen or - on the contrary - weaken the case for astrophysical ALPs. However, it is in any case mandatory to find definitive proof or disproof for these theories in the laboratory as only there uncertainties in experimental parameters of WISP production and identification can be optimally controlled.

To put this into the right context we emphasize that as a rare exception to astrophysical claims in general, testing scenarios of the ALP solution to the transparency anomaly and the anomalous WD cooling is (astonishingly) possible in a small laboratory setup.

\subsubsection{Motivation for hidden photons and minicharged particles}

Hidden sectors, i.e., particles characterized by very weak interactions with ordinary matter, are a very generic feature of field- and string-theory completions of the Standard Model. For example, a hidden sector is a viable instrument to realize supersymmetry breaking. Hidden photons, i.e., gauge bosons of an extra U(1) gauge group, are natural ingredients of these hidden sectors. Most prominently, hidden photons couple to the visible sector via kinetic mixing [46-48], parameterized in the following by $\chi$.

momentum regimes [36]. However, in any case there is strong motivation for purely laboratory measurements, due to some inevitable uncertainties inherent to bounds from astrophysical sources [37].

${ }^{4}$ The broadness of the target region is due to the model-dependent factors which translate the ALP-electron to an ALP-photon coupling. With further studies, a sharpening of the target region should occur. 
Even if in a fundamental theory $\chi=0$, we know from prominent examples within the Standard Model, that effectively $\chi \neq 0$ can be easily induced through integrating out degrees of freedom at higher energy scales ${ }^{5}$, e.g., degrees of freedom arising from string extensions [47, 50, 48]. The $\chi \sim 10^{-8}$ to $10^{-9}$ region, accessible to the first stages of ALPS-II is naturally realized in string compactifications with intermediate string scales [50]. Also, like ALPs, HPs are a viable cold Dark Matter candidate, see [51, 28].

In addition to hidden photons, the hidden sector naturally can also contain matter (scalars or Dirac fermions) with fractional electric charge, called minicharged particles [52, 53]. Most prominently, they can emerge in theories which contain a hidden photon. Search for these particles is crucial, as MCP searches provide an alternative observational window to hidden sectors and in particular can provide insight if the hidden photon turns out to be massless and thus not directly traceable. Here, ALPS-II will chart MCP parameter space which is so-far only indirectly accessible through cosmological arguments.

\subsubsection{Further scientific impact of ALPS-II}

Interestingly, although the new physics models listed above are the most well-reviewed and thus discussed great in detail here, ALPS-II would be sensitive to other, very topical models, which aim at resolving the puzzle of Dark Energy. Only recently, it has been shown that theories of ghostfree massive gravity can indeed be constructed, leading to a broad phenomenology for new scalar degrees of freedom [54]. In particular scalar fields with very light mass are a consequence of using massive gravity to explain the acceleration of the expansion of the universe and thus Dark Energy. For some of these models, it has been pointed out that ALPS-I already provides the best constraints [55] which would be even further improved at ALPS-II or, even better lead to the discovery of Dark Energy particle candidates in a laboratory. However, as this is still a young field, where the model-building roads are not as firmly paved as for WISPs, we will not consider these scenarios here in more detail.

We still want to strongly emphasize that due to these developments, the future scientific impact of ALPS-II could very well be even wider than described in the previous sections.

\subsection{Discovery potential at the three stages of ALPS-II}

ALPS-II is subdivided into three stages, see Sect. 3.1.1 for details. In the following we focus on a theoretical sensitivity analysis for these stages. The installation of the magnet string, required to detect ALPs and MCPs will be realized only in the final stage, ALPS-IIc. In preparation for this final stage, two stages without magnets at different optical cavity lengths will be implemented. $\operatorname{ALPS}-I I a(L=10 \mathrm{~m})$ and ALPS-IIb $(L=100 \mathrm{~m})$ will thus be designed to search for hidden photons.

\subsubsection{Experimental aims in the search for ALPs at ALPS-IIc}

ALP-photon oscillations [56] occur through a pseudo-scalar coupling term $\mathscr{L}_{\text {int,P }}=g_{a \gamma} a \vec{E} \vec{B}$, here enabling the conversion of laser photons characterized by $\vec{E}$ into ALPs $a$. At ALPS-IIc, this happens in an external magnetic field $\vec{B}$ of a HERA dipole magnet string, where the laser photons are

\footnotetext{
${ }^{5}$ One such example is the renowned Heisenberg-Euler effective action [49] leading to effective photon self-interaction through integrating out fermionic degrees of freedom.
} 
polarized in parallel to the magnetic field ${ }^{6}$. The theory of these oscillations for light with frequency $\omega$ has been discussed widely in the literature, see [8] for a recent overview with a focus on LSW setups.

For the envisaged measurements at ALPS-IIc, it is crucial to realize that for a string of $N$ HERA magnets, field-free regions are present at the magnet junctions. Thus the magnetic field is segmented across the entire magnet string. This slightly modifies the formula for the oscillation probability valid for ALPS-I [6]. The corresponding implications for the situation with periodic field-free intersections has been discussed in detail in [57], and one finds that the oscillation probability for photon $\leftrightarrow$ ALP oscillations becomes:

$$
P_{\gamma \leftrightarrow a}=\frac{\omega}{\sqrt{\omega^{2}-m^{2}}} \frac{4 g_{a \gamma}^{2} \omega^{2} B^{2}}{M^{4}} \sin \left(\frac{M^{2} L}{4 \omega N}\right)^{2} \frac{\sin \left(\frac{M^{2} N(L / N+\Delta)}{4 \omega}\right)^{2}}{\sin \left(\frac{M^{2}(L / N+\Delta)}{4 \omega}\right)^{2}},
$$

which reduces to the well-known expression for single magnet setups for $N=1$. Also, we have set $\hbar=c=1$.

In a notation analogous to [6], we have introduced the quantity $M^{2}=\left(m^{2}+2 \omega^{2}(n-1)\right)$, where $n$ is the index of refraction. In addition, $\Delta$ defines the field-free gap-length, where each single HERA dipole has a magnetic length of $l=8.83 \mathrm{~m}$ and the total magnetic length is $L=N l$. For ALPS-IIc, the gap-length is $\Delta=0.936 \mathrm{~m}$ and we envisage the usage of $N=10$ magnets on each side of the light-blocking barrier at field strengths of $B=5.3 \mathrm{~T}$. Details on the setup of the magnet string will follow in Sect. 3.5.

Note that whilst increasing $\omega$ formally increases the conversion probability for WISPs, an appropriate compromise has to be found regarding the photon number flux in front of the wall and the aperture requirements. Hence photon energies beyond the optical regime are disfavored mainly due the lack of appropriate high finesse resonators. For example LSW with X-rays has been performed already [58, 59] but the sensitivity is much smaller than the experiment proposed here, except in the high mass range. In the optical photon energy range, experience at ALPS-I (operated at $532 \mathrm{~nm}$ ) has shown that the mirrors could stand high power intensities only for some 10 hours. We suspect that the single-photon energy of $2.33 \mathrm{eV}$ is sufficient to break chemical bounds which in turn damage the mirror coatings. Such effects have not been observed for $1064 \mathrm{~nm}$ photons, where much expertise and optical component qualification procedures exist in the gravitational wave interferometer community. For this reason, we have switched to $\omega=1.16 \mathrm{eV}$ for ALPS-II. At even lower energies, LSW (with resonant enhancement) is also possible, see ongoing experiments at CERN [60, 61]. However, this option is incompatible with the aperture available in accelerator dipole magnets limiting the sensitivity in ALP searches.

To arrive at the prospective ALPS-IIc exclusion bounds shown in Fig. B, we infer the expected performance based on the results of ALPS-I. Note, that for an LSW-configuration which relies on photon-ALP-photon conversions, it is the square of Eq. 2.1] which determines the experimental sensitivity. In Tab. 1, the respective upgrades are listed and their impact on the testable photonALP coupling $g_{a \gamma}$ is shown to culminate in a total sensitivity gain of about 3082 . Thus, in the most

\footnotetext{
${ }^{6}$ For scalar ALPs, accordingly $\mathscr{L}_{\text {int }, \mathrm{S}}=g_{a \gamma} a\left(\vec{E}^{2}-\vec{B}^{2}\right)$ and only the orthogonal mode of the laser light couples to the external field. For ALPS-IIc, measurements in both polarization modes are foreseen. We emphasize that in the following all statements about the prospective sensitivity for pseudo-scalars apply equally well for the case of scalars.
} 


\begin{tabular}{|l|c|c|c|c|}
\hline Parameter & Scaling & ALPS-I & ALPS-IIc & Sens. gain \\
\hline Effective laser power $P_{\text {laser }}$ & $g_{a \gamma} \propto P_{\text {laser }}^{-1 / 4}$ & $1 \mathrm{~kW}$ & $150 \mathrm{~kW}$ & 3.5 \\
\hline Rel. photon number flux $n_{\gamma}$ & $g_{a \gamma} \propto n_{\gamma}^{-1 / 4}$ & $1(532 \mathrm{~nm})$ & $2(1064 \mathrm{~nm})$ & 1.2 \\
\hline Power built up in RC $P_{\mathrm{RC}}$ & $g_{a \gamma} \propto P_{r e g}^{-1 / 4}$ & 1 & 40,000 & 14 \\
\hline$B L$ (before\& after the wall) & $g_{a \gamma} \propto(B L)^{-1}$ & $22 \mathrm{Tm}$ & $468 \mathrm{Tm}$ & 21 \\
\hline Detector efficiency $Q E$ & $g_{a \gamma} \propto Q E^{-1 / 4}$ & 0.9 & 0.75 & 0.96 \\
\hline Detector noise $D C$ & $g_{a \gamma} \propto D C^{1 / 8}$ & $0.0018 \mathrm{~s}^{-1}$ & $0.000001 \mathrm{~s}^{-1}$ & 2.6 \\
\hline Combined improvements & & & & 3082 \\
\hline
\end{tabular}

Table 1. Parameters of the ALPS-I experiment in comparison to the ALPS-II proposal. The second column shows the dependence of the reachable ALP-photon coupling on the experimental parameters. The last column lists the approximate sensitivity gain for axion-like particle searches compared to ALPS-I. The main sensitivity gain is due to the enhanced magnetic length arising from the $2 \times 10$ HERA-dipole magnet string, cf. Tab. 3 for details. A sizable additional gain arises from the installation of a regeneration cavity (RC), cf. Sect. 3.2 for details. A further upgrade comes also from the detection side. The numbers given in this table are for a transition edge sensor detector, as this is the detector which is expected to be used in the final version of all three stages of ALPS-II, see Sect. 3.4 and Tab. 2 For hidden photons, there is no gain from the magnetic field. Thus the sensitivity gain follows as above except for the factor coming from $B L$ and amounts to 147 for hidden photons.

sensitive low-mass region $m \lesssim 10^{-4} \mathrm{eV}$ the explored coupling values at ALPS-I $g_{a \gamma} \gtrsim 7 \times 10^{-8}$ $\mathrm{GeV}^{-1}$ will be extended to $g_{a \gamma} \gtrsim 2 \times 10^{-11} \mathrm{GeV}^{-1}$ at ALPS-IIc. For a recent comprehensive overview of the ALP parameter space also at larger masses, see, e.g., [27, 28, 2]].

As visible in Fig. B, for masses above $m \gtrsim 10^{-4} \mathrm{eV}$, the sensitivity curve adopts an oscillatory behavior. Physically this corresponds to phase-mismatches in the ALP production. This is of course unwanted, as ALPs could very well just hide in these regions where the sensitivity is decreased. In order to fill these gaps in sensitivity, one has the possibility to shift the effective photon mass by the insertion of gas into the beam tube. This method has already proven to be very successful at ALPS-I using Argon [6].

For a single magnet configuration $N=1$, it is easy (and analytically possible) to spot the minima of the transition probability in vacuum. The gas index of refraction which optimally closes the gaps can then be computed by minimizing the expression in Eq. 2.1 with respect to $(n-1)$, see Eq. 2.3 below. For $N>1$, all gaps will be covered ideally through a parameter scan with different gas pressures through the insertion of Helium, as detailed in Sect. 3.6.

In Fig. B, we plot the transition probability for vacuum and exemplarily for one single Helium pressure corresponding to $0.017 \mathrm{mbar}$ (see paragraph including Eq. 3.3 for details on the index of refraction to pressure relation).

To conclude, ALPS-IIc charts thus-far unexplored parameter space, surpassing even current helioscope limits at low masses and, for the first time, simultaneously tackles regions which are favored by theory (Dark Matter, ALPs from intermediate scale string theory) as well as astrophysical hints (WD cooling, TeV transparency), cf. Fig. 目. 


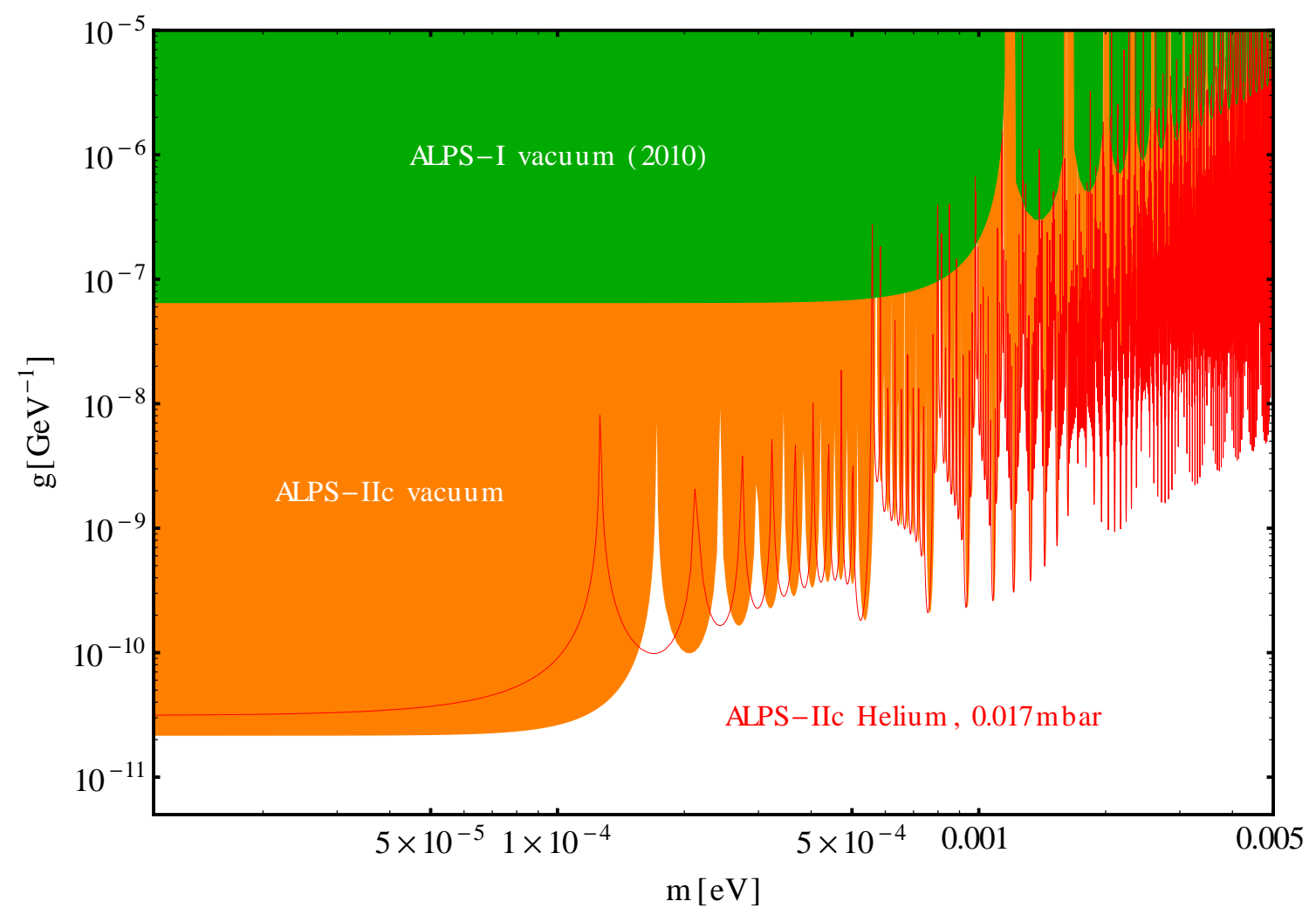

Figure 3. Prospective sensitivity reach for axion-like particles in the final stage of the ALPS-experiment, ALPS-IIc. The uppermost, green shaded area gives the currently most sensitive laboratory bounds on ALPs as reported in [0] for comparison. Below, in orange, we give the expected sensitivity reach of ALPS-IIc, surpassing the currently most sensitive laboratory limits by more than three orders of magnitude, see main text for details. In addition we give exemplarily the sensitivity reach with Helium gas at a fixed pressure of 0.017 mbar. With varying pressures, the Helium insertion allows to cover the sensitivity gaps of the vacuum measurements and thus scan the entire accessible parameter range. For clarity, parameter regions favored by theory and astrophysics hints are shown in Fig. 目 only.

\subsubsection{Discovery potential for HPs and MCPs at ALPS-II}

\section{Projected Sensitivity in the Search for Hidden Photons}

The theory of photon-hidden photon oscillations differs from the case of photon-ALP-oscillations most prominently by the fact that former also occur without an external magnetic field (both particles have equal spin). If HPs have a mass, kinetic mixing behaves as mass mixing and thus photonHP oscillations occur (in analogy to oscillations among different neutrino flavors) [62]. This leads to the disappearance and regeneration of photons as they propagate in vacuum and makes their discovery particularly easy in light-shining-through a wall setups.

Photon-HP oscillations in LSW-experiments are reviewed in [8] and the oscillation probability 
for one oscillation (in either direction) $\gamma \leftrightarrow \gamma^{\prime}$ is given as [63]

$$
P_{\gamma \leftrightarrow \gamma^{\prime}} \simeq 4 \chi^{2} \frac{m_{\gamma^{\prime}}^{4}}{M_{\gamma^{\prime}}^{4}} \sin ^{2}\left(\frac{M_{\gamma^{\prime}}^{2} L}{4 \omega}\right),
$$

where, in analogy to the definition for ALPs $M_{\gamma^{\prime}}^{2}=\left(m_{\gamma^{\prime}}^{2}+2 \omega^{2}(n-1)\right)$. Again, for the LSW-setup of ALPS-II, the square of Eq. 2.2 is the relevant quantity.

Figure 7 shows the prospective exclusion limits obtained in the various ALPS-II stages. Note that at each single stage, ALPS-II will chart thus-far unexplored HP parameter space, implying that at each stage a particle discovery is indeed possible. The black vertical dashed lines in Fig. 7 refer to the Dark Radiation hint [64] derived from CMB data [65, 66]. Although the newest Planck results [67] still contain indication for an extra relativistic degree of freedom in the CMB (although reduced to $N_{\text {eff }}=3.36 \pm 0.34$ at $68 \%$ confidence), the hidden photon is unlikely to be under the viable candidates to account for this phenomenon. It has been shown that, the total production of hidden photons in the sun has been underestimated, since the production of longitudinal modes has not been taken correctly into account. Following [68, 69], the parameter space above the line labeled "Longitudinal" in Fig. 7 is already excluded by the bounds on the energy loss due to hidden photon production in the sun's plasma. However, one should note that for some models the bounds labeled "Longitudinal" might not apply, e.g. in models, where the hidden photon decays. In a situation where, for example a sizable amount of hidden photons from the sun cannot reach earth due to decay, a laboratory search is indispensable. This holds true also for limits on HPs from direct Dark Matter searches [70].

Our measurements will nicely complement the exclusion limits derived from CAST helioscope measurements [24] as well as regions covered by measurements of Coulomb's law [71] and cosmological implications [72]. For a recent comprehensive overview of the hidden photon parameter space, see, e.g., [27, 28].

The prospective exclusion limits for the different vacuum stages of ALPS-II are estimated as follows. In contrast to the ALPS-I setup[[6], infrared lasers with $\omega=1.16 \mathrm{eV}$ will be used and the length of the vacuum tubes $L$ is increased ${ }^{7}$. Thus, quite generally, as the argument of the sine depends on $\mathrm{Lm}^{2} / \omega$, the set-in of the oscillatory behavior within Eq. 2.2 is shifted to lower mass-values for enhanced lengths. The frequency of our laser sets also the mass scale up to which our experiment is sensitive to hidden photons. At higher masses, HPs are well probed through helioscopes and their production in the sun and horizontal branch stars, see, e.g. Fig. 6-3 in [27]. The HP coupling to electrons is particularly well accessible at accelerators, see, e.g. Fig 6-2 of [27] for an overview.

Quantitatively, the expected increase in the sensitivity to $\chi$ can be also estimated from Tab. [1. The crucial difference to the situation with ALPs is that the "magnetic length" $B L$ will not give an enhancement factor as photon-HP oscillations do not rely on external fields. Rather, the main sensitivity gain will arise from the use of a regeneration cavity. Note that the resonant regeneration

\footnotetext{
${ }^{7}$ Note that for ALPS-I, the length of the vacuum tubes was slightly different in the production- and regenerationregions, leading to a "non-saturation" of the exclusion limits for at $m_{\gamma^{\prime}} \gtrsim 10^{-3} \mathrm{eV}$. E.g., this led to a minimum of an oscillatory envelope function at about $m_{\gamma^{\prime}} \approx 2 \times 10^{-3} \mathrm{eV}$, cf. the green shaded area in 4 . For ALPS-II however, the length of the vacuum tubes on the generation and regeneration side will be equal and such unwanted features are avoided.
} 
principle has been tested with microwaves in the sub-quantum regime [73]. An early optical setup is described in [74].

For ALPS-IIa with production and regeneration cavity as well as the transition edge sensor, the enhancement factor with respect to $\chi$ is 147 . ALPS-IIb will further enlarge the covered parameter space by the length increase $L=100 \mathrm{~m}$ and a corresponding shift in the covered mass range to lower masses. These statements are summarized within the Fig. [4, showing the ALPS-II sensitivity in the hidden photon parameter space. In addition, ALPS-IIb will begin to tackle the HP cold DM region. Note that recent [75] and upcoming helioscope searches (SHIPS [76]) are sensitive in particular for $m_{\gamma^{\prime}}>10^{-2} \mathrm{eV}$.

As visible from Fig. $\sharp$, cusps appear in the sensitivity range for HP masses $m_{\gamma^{\prime}} \gtrsim 10^{-3} \mathrm{eV}$, corresponding to phase-mismatches in the HP production in the vacuum measurements. These minima lie at points where $m_{\gamma^{\prime}}^{2} L / 4 \omega=s \pi$, with $s \in \mathbb{N}^{+}$. A viable method to close these gaps in the sensitivity reach is the variation of the effective photon mass through insertion of gas into the tubes. This slightly decreases the amplitude of the conversion probability.

To compute index of refraction which optimally closes the gaps, one has to minimize the expression in Eq. 2.2 with respect to $(n-1)$. Thus, defining $y=M_{\gamma^{\prime}}^{2} L /(4 \omega)$, the transition maxima lie at $^{8}$ solutions to a condition with infinite but numerable solutions

$$
\tan (y)=y \Leftrightarrow y=\{0,4.49,7.73 \ldots\}
$$

To cover the lowermost gap in the sensitivity for ALPS-IIa $(L=10 \mathrm{~m})$ and ALPS-IIb $(L=100 \mathrm{~m})$, indices of refraction of $(n-1)=4.57 \times 10^{-8}$ and $(n-1)=3.52 \times 10^{-9}$ are optimal, respectively. As $s \rightarrow \infty$, the best indices of refraction adequate to cover the gaps optimally shift to $(n-1)=$ $5.31 \times 10^{-8}$ and $(n-1)=4.43 \times 10^{-9}$, respectively. Thus for ideal coverage of the gaps, for ALPS-IIa we should have $(n-1) \simeq 5 \times 10^{-8}$ and for ALPS-IIb $(n-1) \simeq 4 \times 10^{-9}$. This choice is depicted in Fig. 6 . As can be seen, the gas insertion technique, also pioneered by ALPS-I [6] will enable to cover the higher mass reach entirely.

To conclude, the HP search at ALPS-II will not only cover a parameter space not accessible by astrophysical considerations, but also tackle a kinetic mixing region naturally predicted by string compactifications with intermediate string scales as well as a fraction of the HP DM parameter space.

\section{Projected Sensitivity in the Search for Minicharged Particles}

Light-shining-through a wall for minicharged particles at ALPS-IIc comes about as follows: Photons can be converted into (massless) hidden photons through a virtual MCP loop within an external magnetic field. Subsequently, the hidden photons can traverse the barrier as in Sect. 2.2.2 and can thereafter be reconverted into photons through the intermediate MCP loop [62].

The corresponding conversion probability for this process reads [77]

$$
P\left(\gamma \leftrightarrow \gamma^{\prime}\right) \simeq\left|\frac{m_{\tilde{\gamma}^{\prime}}^{2}}{M^{2}}\right|\left|e^{i k_{+} L}-e^{i k_{-} L}\right|^{2},
$$

\footnotetext{
${ }^{8}$ Note that in [6] the theoretical derivation had a minor fault which however has no influence on presented results therein.
} 


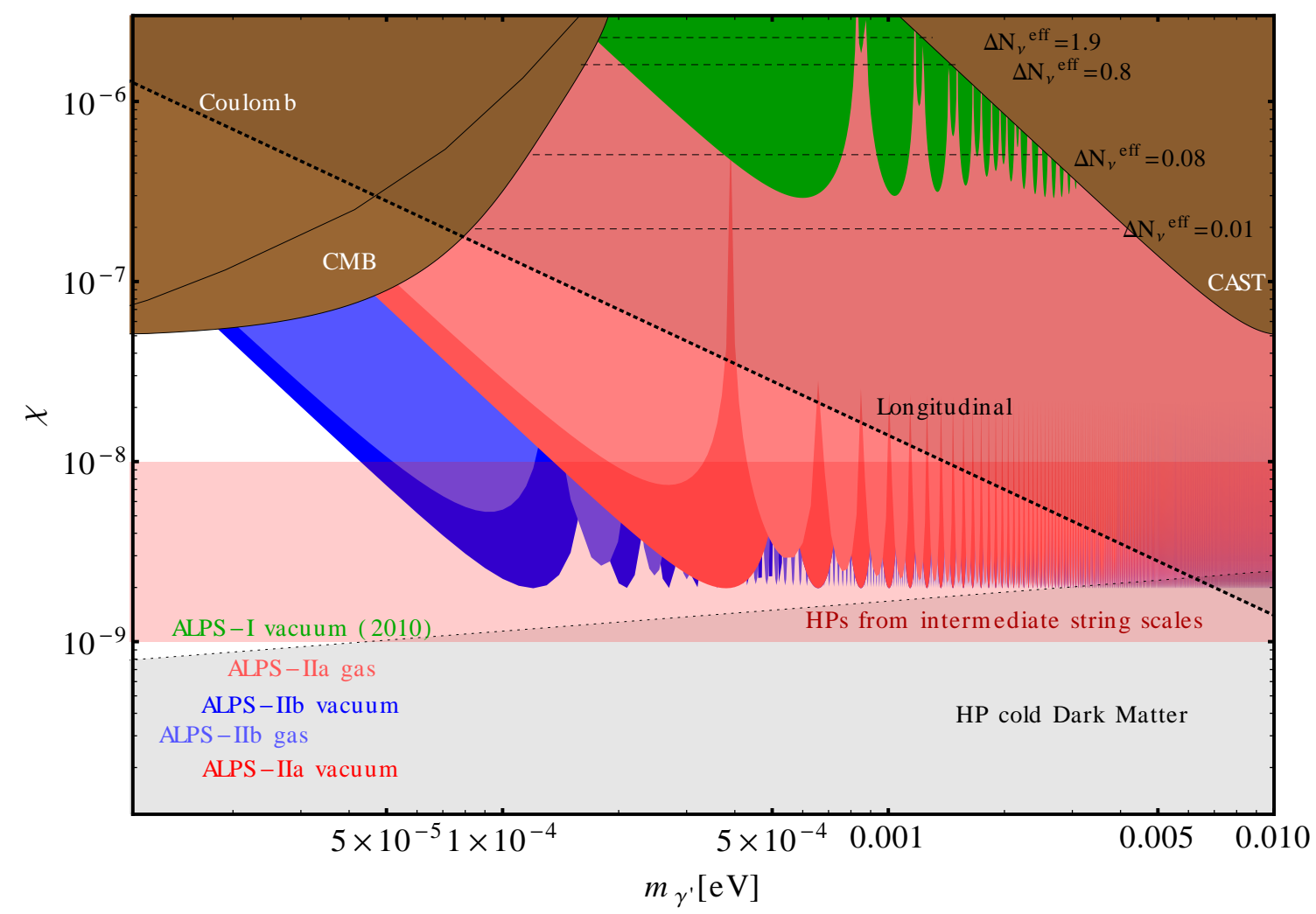

Figure 4. Coupling-mass plane for hidden photons. The shaded areas indicate the prospective parameter reach for HPs at ALPS-IIa with regeneration cavity and ALPS-IIb in setups with vacuum and gas-filled tubes, respectively. The uppermost, green shaded oscillatory curve denote the results obtained by ALPS-I in the vacuum case for comparison. Below, in light and dark red, the parameter reach for ALPS-IIa with regeneration cavity and gas/no-gas is indicated. From there, the light and dark blue areas to the left denotes the reach of ALPS-IIb, with and without gas, respectively. For the parameter choices with gas, see main text. The black dashed lines enclose the HP Dark Radiation hint [64], however recent analyses [68, 69] exclude the parameter range above the line labeled "Longitudinal, thus disfavoring this interpretation. The gray shaded lower area indicates where HPs could be cold DM [28]. Note that the $\chi \sim 10^{-8}$ to $10^{-9}$ region is naturally realized in string compactifications with intermediate string scales [50] (beam-like red-shaded area). The brown areas in the upper right and left corners denote exclusion bounds from other experiments, see text for details.

$$
k_{ \pm}=\frac{1}{4 \omega}\left(2 \omega^{2}(n-1)-m_{\tilde{\gamma}^{\prime}}^{2} \pm M^{2}\right)\left(1 \pm \chi^{2} m_{\tilde{\gamma}^{\prime}}^{2} / M^{2}\right)
$$

where now $m_{\tilde{\gamma}^{\prime}}$ is the hidden photon effective mass (accounting for the MCP-induced change in the dispersion relation). It can be written as $m_{\tilde{\gamma}^{\prime}}^{2}=-2 \omega^{2} \Delta N\left(\varepsilon, B, m_{\mathrm{MCP}}\right)$ with $\Delta N\left(\varepsilon, B, m_{\mathrm{MCP}}\right)$ being the complex index of refraction due to minicharged particles of charge $\varepsilon$ and mass $m_{\mathrm{MCP}}$. The minicharge $\varepsilon$ is related to the kinetic mixing parameter $\chi$ of the hidden photons through $\chi e_{\mathrm{H}}=\varepsilon e$, where $e_{\mathrm{H}}$ is the scalar or Dirac spinor coupling in the hidden sector and $e$ the ordinary electromagnetic coupling. The explicit expression for $\Delta N$ for scalar or Dirac spinor MCPs is given in Ref. 62]. 
In a scenario where $e_{\mathrm{H}}$ matches the coupling in the visible sector ${ }^{9}$, ALPS-II can improve the best limits provided by ALPS-I by around two orders of magnitude. This is due to the fact that the transition probability scales as $B^{2 / 3}$ in the low-mass limit ${ }^{10}$, and the sensitivity enhancement factor presented for ALPs (cf. Tab. (1)) is reduced accordingly.

As a consequence, ALPS-II will chart MCP parameter space which is so-far only indirectly accessible through cosmological or astrophysical arguments [82]. For a recent comprehensive overview of the MCP parameter space, see, e.g., [27].

\section{The experiment}

Here we describe the experimental setup starting with a summary of its main characteristics. For a short summary see also [83].

\subsection{Overview}

This section gives a brief overview on the different stages of the ALPS-II experiment and sketches its operation modes which will allow us to verify the achieved sensitivity and, eventually, to pin down the nature of the WISP responsible for light shining through the wall.

\subsubsection{Stages of the experiment}

It is planned to realize the ALPS-II experiment in three main stages. This will allow for a thorough development of the required new techniques without the necessity of large infrastructure efforts (i.e., long magnet strings) right from the beginning. As we plan to enter new territory in parameter space as well as in optical setups, the staged approach is also advantageous because it allows to adapt to unforeseen new developments. We will show below, that all three stages will provide sensitivities for different kinds of WISPs beyond present day laboratory experiments allowing for interesting physics results, see also Sect. 2.2.

It should be stressed that the ALPS collaboration considers the potential technological risks as manageable as will be shown in this document, but the staged approach described here is also very well compatible with DESY's commitments in other larger projects.

The basic three stages of ALPS-II are shown in Fig. 5.

- ALPS-IIa:

This stage aims for demonstrating all features of the optical system and the superconducting transition-edge sensor (TES) detector in a dedicated laser laboratory with $10 \mathrm{~m}$ long production and regeneration cavities. ALPS-IIa itself will be subdivided into several steps. A $1 \mathrm{~m}$ tabletop experiment in air with reduced optical power and cavity finesses is already being conducted, which will allow to prove the consistency of the optical design, develop and test the control systems and yield first performance data of the overall system. Subsequently, the high power production cavity will be realized allowing for a first data run using the CCD

\footnotetext{
${ }^{9}$ Although probing MCPs in an LSW setup with direct access to the MCP coupling is possible, this is not an immediate option for ALPS-II, as in this situation a solenoidal magnetic field configuration is preferred [78, 79].

${ }^{10}$ This is a consequence of the strong-field limit of the polarization tensor, applicable for minicharged particles 80 , 81.
} 


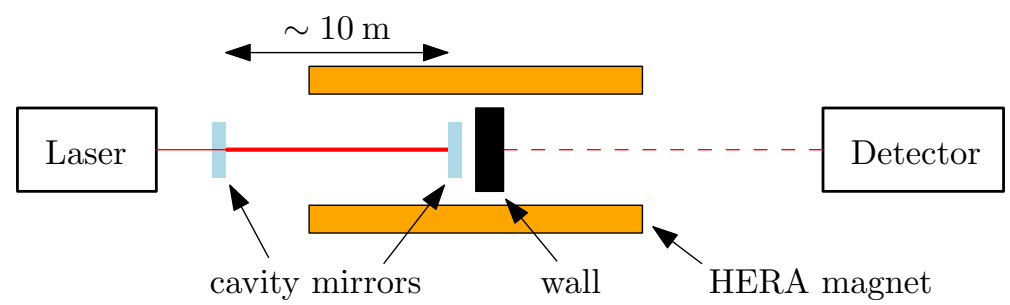

(a) ALPS-I

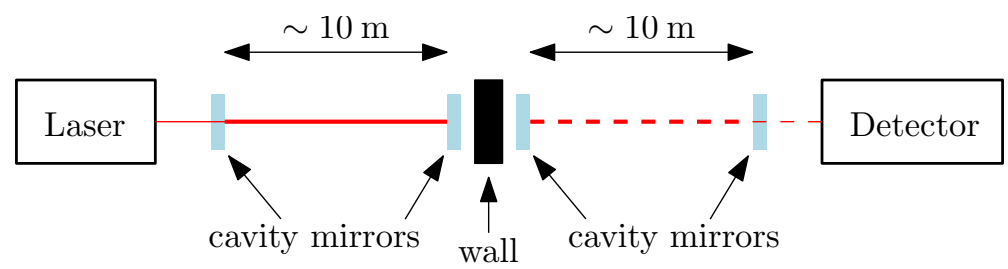

(b) ALPS-IIa

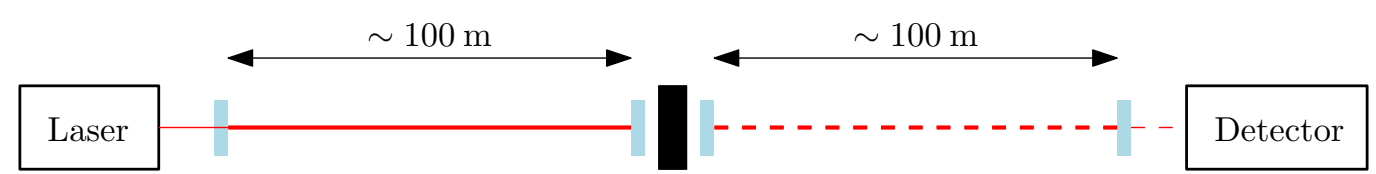

(c) ALPS-IIb

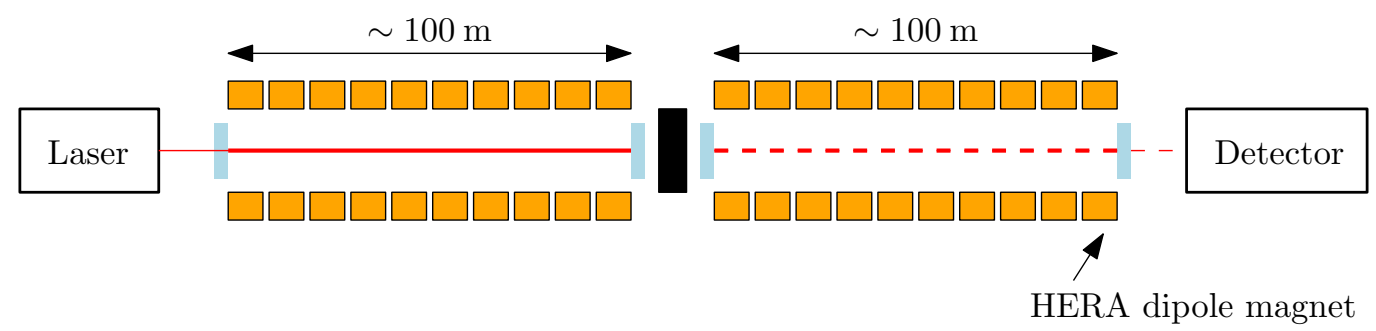

(d) ALPS-IIc

Figure 5. Schematic view of the different phases of ALPS-II and of ALPS-I. aLPS-I with a $\sim 10 \mathrm{~m}$ long production cavity and cavity end mirror and wall inside of a HERA superconducting dipole magnet. [b] ALPS-IIa: prototype with two $10 \mathrm{~m}$ long cavities. [C] ALPS-IIb: prototype with two $100 \mathrm{~m}$ long cavities. (d) ALPS-IIc: prototype with two $100 \mathrm{~m}$ long cavities using the HERA superconducting dipole magnets.

camera and perhaps already the TES detector to search for hidden photons. This sub-step is expected to be concluded in early 2013. Afterwards, the regeneration cavity including all features of the final ALPS-IIc stage will be set up. In 2014, we plan to search for hidden photons with full sensitivity. Besides interesting physics results, this experiment corresponds in all aspects to ALPS-IIc, having only a shorter length and lacking magnets. By the middle of 2014, we also envisage to have sufficient experience with the straightening of HERA dipoles so that all major ingredients of the final ALPS-II stage are known.

It should be mentioned that most costs of ALPS-IIa have been already covered. The new laser laboratory has been constructed in 2011. We are reusing the laser of ALPS-I. Nearly all optical components as well as the vacuum system have been purchased already. 


\section{- ALPS-IIb:}

In principle this stage corresponds to ALPS-IIa, but with about $100 \mathrm{~m}$ long cavities. It will be installed in one straight section of HERA (around the experimental hall HERA-West) using the long straight vacuum pipe of the HERA proton ring. This second stage will demonstrate the functionality of the system on the length scale foreseen for the final setup with HERA dipole magnets as well as the ability to cope with the situation in the HERA tunnel, e.g., seismic vibrations (which are expected to be smaller than in the laser laboratory of ALPSIIa), cleanroom installation, and operation given the tight spatial constraints. The aperture boundaries are a little relaxed compared to ALPS-IIc, so that setting up and operating the experiment will allow us to gain valuable experience for the final ALPS-IIc setup.

The experience with ALPS-IIb should allow for a fast realization of ALPS-IIc. As ALPS-IIb will reuse many components of ALPS-IIa the additional costs will be moderate. Mainly new infrastructure, new cleanrooms and new mirrors for the long cavities have to be provided. Construction for ALPS-IIb could start in the second half of 2013 with preparation of the site and installation of the cleanrooms. This should allow to install the optics and the detector starting late 2014 being ready for data taking in the second half of 2015 .

\section{- ALPS-IIc:}

This is the final version of ALPS-II with full length cavities and straightened HERA dipoles. If the approval of this stage is given in the middle of the year 2014, site preparations of a straight section in the HERA tunnel (most likely at HERA North) could start in the beginning of 2015, when the construction of the linear accelerator of the European XFEL project is completed. Note that much more effort is required here compared to ALPS-IIb as the site is to be prepared to accommodate 20 straightened HERA dipole magnets, see Fig. 6. The time schedule for the installations strongly depends on the available manpower of the DESY infrastructure groups involved. At present, it is estimated that both the site preparation and the installation of the magnets (which are to be straightened in 2014) will require about one year so that ALPS-IIc will be ready for commissioning and data taking in the year 2017. At the end of the same year, physics results will be available.

In Tab. 1 the main parameters and the sensitivity of ALPS-I and ALPS-IIc are compared. We aim for surpassing the present laboratory sensitivity in the coupling constant of axion-like particles to photons by more than three orders of magnitude. In the low mass regime we aim to even surpass bounds provided by helioscope measurements, cf. Fig. Q2. DESY, coincidentally, seems to be an ideal place for such an enterprise. First, the HERA dipole magnets will possibly allow for a relatively easy and cheap way of straightening to increase their aperture. This aperture allows to set up ALPS-IIc with up to 24 magnets given the lower bound of tolerable clipping losses. Second, this setup just fits into a straight section of the HERA tunnel, so that ALPS-IIc does not require any new building. Third, and to finalize the number of lucky coincidences, 24 spare HERA dipole magnets are available at DESY so that the construction of ALPS-IIc does not demand demounting of the arcs of the HERA accelerator (besides using the cryoboxes as discussed later).

\subsubsection{ALPS-II operation modes and signal interpretation}

While operating the experiment the following main issues have to be guaranteed: 


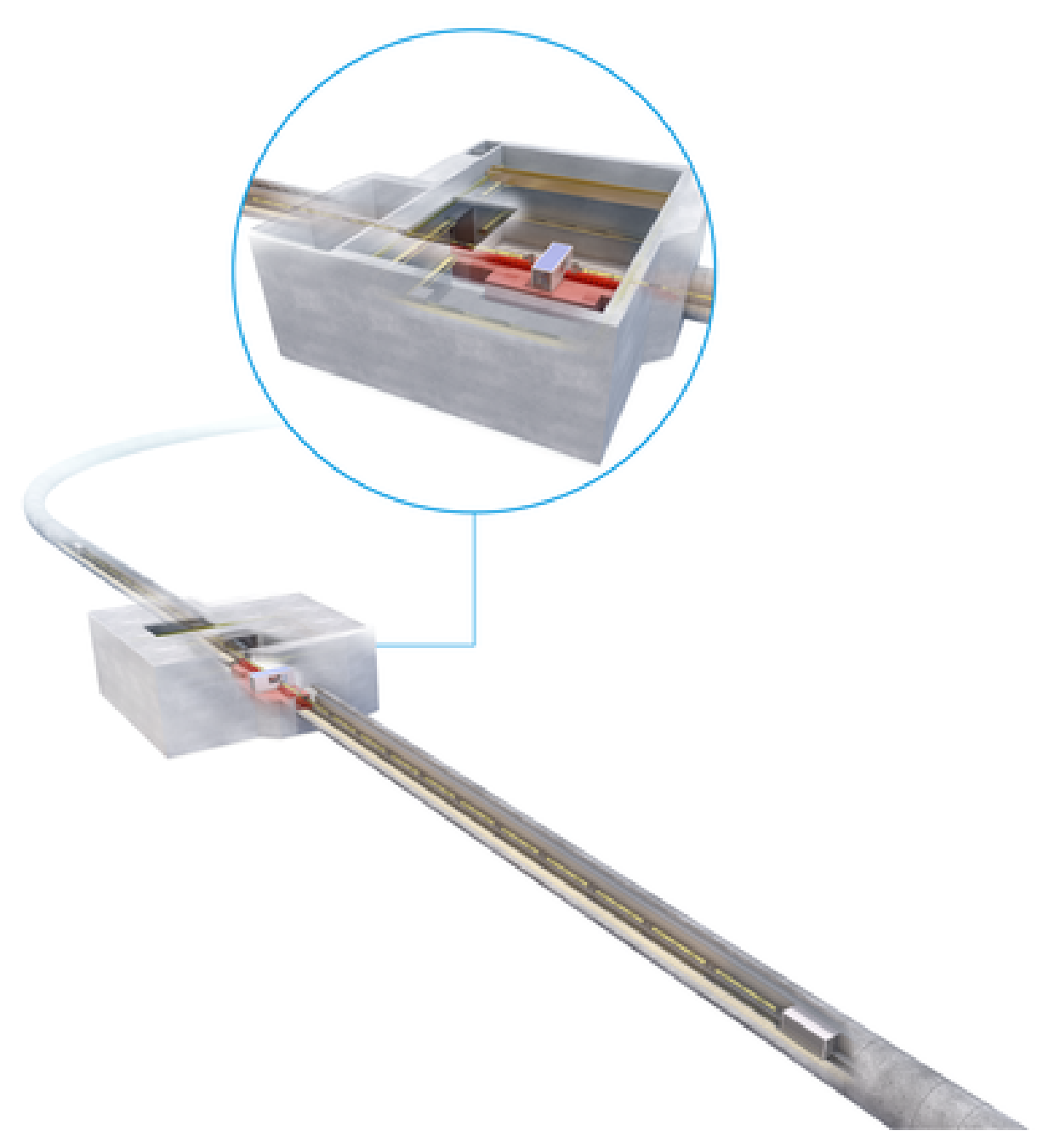

Figure 6. Artist's view of the ALPS-IIc setup. The picture shows a straight section of the HERA tunnel equipped with 20 HERA dipoles. The middle part, accommodating the central breadboard including the "wall" is highlighted.

- the alignment of production and regeneration cavity,

- the power build-up for $1064 \mathrm{~nm}$ light in the regeneration cavity,

- and the pointing of regenerated light from WISPs onto the detector.

All these critical items can be probed by opening a shutter in the wall separating the production and regeneration part of the experiment. The shutter is shown on the central breadboard in Fig. 12, allowing a tiny fraction of the infrared light of the production cavity to enter the regeneration 
cavity. If both cavities are aligned and the resonance condition of the regeneration cavity is met, the quadrant photodiodes at the end of the regeneration cavity (see Fig. 12) will notice the expected intensity of $1064 \mathrm{~nm}$ radiation. At the same time, the detector will sense the infrared light in the regeneration cavity. As this light moves exactly along the same path as photons from reconverted WISPs, the pointing can be checked easily. However, when the shutter is opened, about $1.7 \mathrm{~mW}$ of $1064 \mathrm{~nm}$ light ( $10^{16}$ photons per second) will reach the detector. This intensity is to be damped by dedicated filters to allow the detectors to cope with the flux.

The analysis of the data will rely on a thorough understanding of any backgrounds to detect a potentially very small photon flux from reconverted WISPs. A signal will be identified by comparing data taken under conditions where WISPs can be expected (so-called "data frames") with data which by construction cannot contain reconverted WISPs ("dark frames"). To minimize changes in the experimental set-up for data and dark frames the regeneration cavity (for the ALPS phases IIb and IIc) will be detuned to not amplify $1064 \mathrm{~nm}$ radiation while staying in lock for $532 \mathrm{~nm}$ light. This can be achieved with the help of the AOM on the central breadboard (see Fig. 12 in the optics section), again checked by opening the central shutter. With the regeneration cavity being ineffective for photons from WISPs, their flux at the detector will be damped by the power build-up factor (about 40000) of the regeneration cavity. Hence, these dark frames correspond to background runs without any WISPs, while the ambient conditions (i.e., stray light, fluorescence effects) remain unchanged. Of course, additional test data, where, for example, the main laser is shut off, the frequency doubled green light suppressed or the detector totally blocked, will be taken to understand the origin of any spurious background photons. Experience at ALPS-I showed that continuous improvements and investigative searches resulted finally in well understood remaining backgrounds (essentially only from detector effects).

Surely, a discovery of WISPs by a photon signal at ALPS-II is to be proven by systematic studies even if the statistical significance is beyond doubts. In addition, such studies will allow to pin down some properties of the detected WISP. The following list gives an overview on possibilities to test the WISPy nature of a photon excess:

- For any WISP the signal is expected to rise with the power build-up in the regeneration cavity. This prediction can be easily probed by changing the lock condition for the regeneration cavity.

- A comparison of runs with magnets on and off allows to discriminate between WISPs which mix kinetically with photons (hidden photons) and WISPs which couple to more than one photon (like ALPs).

- The polarization of the laser light can be changed with respect to the direction of the magnetic field. Hence, the parity of a WISP, which shows only up in data with magnets on, can be determined. Scalars are produced with the polarization perpendicular to the magnetic field, pseudo-scalars with a polarization parallel to the field.

A production not strongly depending on these polarization directions might indicate the existence of minicharged particles and massless hidden photons.

- In Figs. 3 and 4 the dependence of the WISP production and reconversion on the gas pressure within the cavities is shown. This effect would allow to estimate the mass of the discovered 
WISP, ranging from relatively accurate measurements, if the mass is sufficiently large, to upper limits for very lightweight WISPs.

In summary, the ALPS-II setup will allow for many different systematic checks to prove the origin of a photon excess and to even determine properties of a WISP particle. The sketched run procedure also enables sufficient tests to monitor the performance of the experiment, so that a WISP signal (in the accessible sensitivity regime) cannot escape detection.

\subsection{Laser and optics}

The main goal of the optical design of ALPS-II is to make the electro-magnetic field provided by the laser beam on one side of the wall as large as possible and to detect a possibly regenerated field on the other side with a very high sensitivity. Both tasks can be supported with optical Fabry-Perot type resonators. On the side in front of the wall, the ALPS-II production cavity (PC) can increase the optical power of the light beam directed towards the wall by a factor of 5000 compared to the power of the injected laser. Behind the wall, the regeneration cavity (RC) increases the production probability with which photons are created from the axion field [84, 85] by a factor of 40000 . Please note that operating the cavities requires the usage of a continuous wave laser.

\subsubsection{Technical challenges}

These main goals translate into several requirements on the optical system that have to be fulfilled. Two high finesse optical cavities have to be operated within the small aperture provided by the magnet string with identical optical axes. Both cavities have to be resonant for the same light frequency. The mirrors have to be controlled to form stable eigenmodes co-linear to the central axes of the magnets. The laser beam has to be matched to these modes spatially as well as concerning its frequency. While the control of the laser and the PC is state-of-the-art in several fields of modern optics, e.g., the interferometric detection of gravitational waves [86], a new challenge arises in controlling the RC. This cavity has to be kept aligned and resonant for the regenerated light without the use of any control light in the spatial and spectral acceptance range of the ALPS-II detector.

\subsubsection{Conceptual design}

The design goal of ALPS-II is to inject $30 \mathrm{~W}$ into the PC with a power buildup of 5000 and to operate the $\mathrm{RC}$ with an equivalent power buildup of 40000. The injection stage of the PC as of August 2012 is shown in Fig. 7 . The main limitations for the optical design are given by the aperture of the magnet strings, by the durability and losses of available dielectric mirror coatings and by the environmental disturbances, namely the vibrational fluctuations and their coupling into the length degree of freedom of the optical cavities. To get a quantitative understanding of these design boundaries we use a staggered experimental approach: We first set up a phase ALPS-IIa prototype experiment with $10 \mathrm{~m}$ long cavities to understand the limitations from dielectric coatings and gain experiences in controlling the two cavities. Sensor and actuator range and noise investigations as well as lock-acquisition studies are part of this phase. Furthermore, the analysis of vibrations and other environmental disturbances will be an important outcome of this phase.

Based on the information gained, we will modify the setup to allow an operation of two $100 \mathrm{~m}$ long cavities in phase ALPS-IIb. This phase will not have the small aperture set by the magnets, 


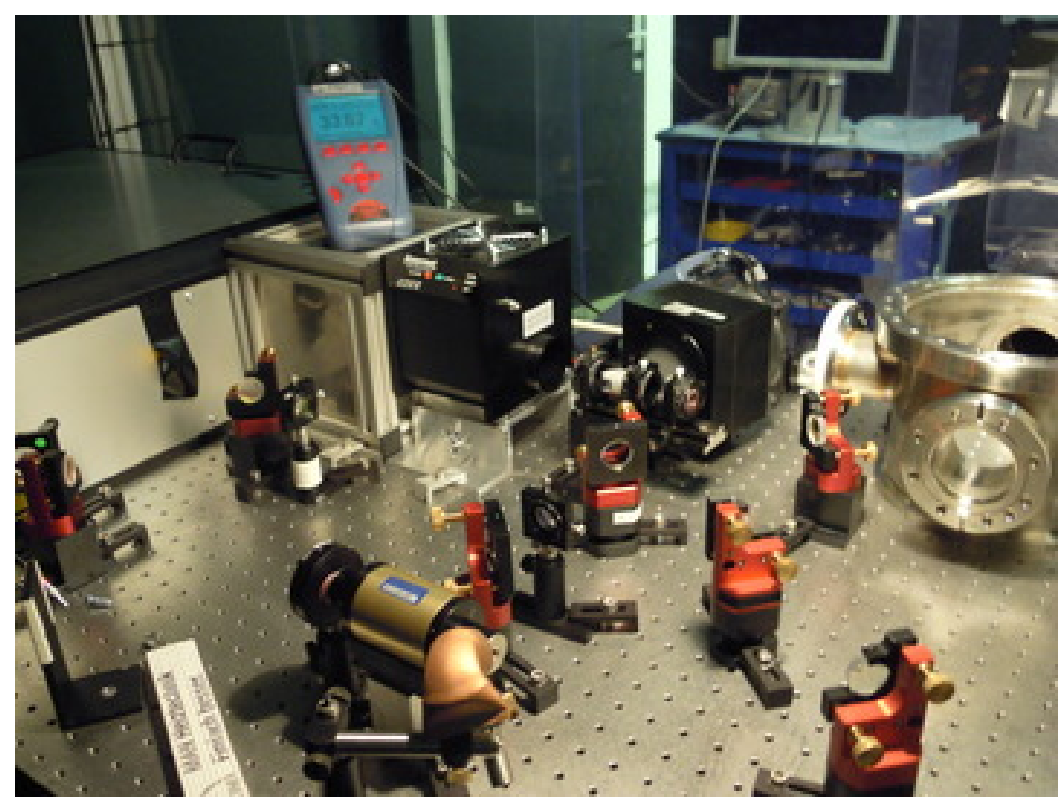

Figure 7. This picture shows the injection stage of the production cavity in the laser laboratory in hall 50 at DESY.

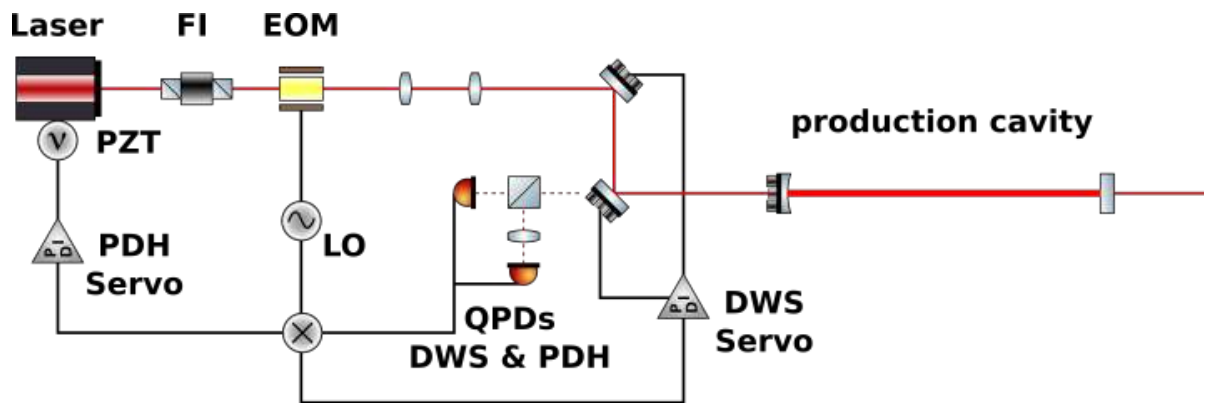

Figure 8. Schematic of the ALPS-II injection stage including the production cavity.

which leads to somewhat relaxed requirements for the cavity alignment control. Once, both $100 \mathrm{~m}$ long cavities are operable, remaining fluctuations can be measured and optionally reduced. Furthermore, we can measure the margin in control authority and bandwidth to estimate the acceptable level of additional vibrations related to magnet operation and environmental noise. These important results will feed into the final design of the ALPS-IIc phase, in which the two cavities need to be operated in the small aperture given by the magnets. The three different phases of the experiment are schematically shown in Fig. 5.

A schematic sketch of the laser and the PC is shown in Fig. 8. We will use a $35 \mathrm{~W}$ high-power single-mode single-frequency laser system operating at $1064 \mathrm{~nm}$. The master-oscillator poweramplifier design allows to amplify the power of the $2 \mathrm{~W}$ master laser (Innolight Mephisto) to $35 \mathrm{~W}$ while maintaining the frequency stability of the low noise master laser. Several of these lasers are currently in use in gravitational wave detectors and have proven to be reliable with low maintenance efforts for many years. This laser was already successfully used in the ALPS-I experiment. The laser light passes a Faraday isolator (FI) to avoid back reflections into the laser and an electro- 
optical modulator that imprints phase modulation sidebands onto the light. These sidebands are used in a heterodyne detection scheme (Pound-Drever-Hall (PDH) sensing, [87]) to gain information on the deviation of one of the resonance frequencies of the PC from the laser frequency. Two lenses and two steerable piezo-electric transducer (PZT) mirrors are used to match the Gaussian laser beam to the eigenmode of the PC. A fraction of the light reflected by the cavity is transmitted by a turning mirror and detected with two QPD that have a detection area split in four quadrants. A lens is used in front of one quadrant photodiode (QPD) to shift the Gouy phase of the Gaussian beam by $90^{\circ}$. Via the heterodyne differential wavefront sensing (DWS) scheme [88], we will gain information on the parallel shift and tilt of the laser beam with respect to the cavity's optical axis, both in the horizontal and vertical plane. The sum of all quadrants of one of the QPDs is used in the PDH sensing.

By appropriately feeding the alignment and length error signals back to the laser frequency control actuator and to the PZT mirrors we can stabilize the high power laser beam to match the cavity eigenmode and to keep laser and cavity resonant. The goal is to keep the remaining spatial and frequency fluctuations small enough to limit the fluctuations of the power buildup in the cavity to less than 5\% (RMS).

The optical parameters of the PC and RC are designed to allow for high buildup factors. To reduce the risk of mirror damage we limited the maximum intensity on the $\mathrm{PC}$ mirrors to $500 \mathrm{~kW} / \mathrm{cm}^{2}$ (well below the damage threshold of about a few $1000 \mathrm{~kW} / \mathrm{cm}^{2}$ for sputter and e-beam coatings), a level at which mirrors in the gravitational wave community have been operated for long durations without degradation. For a smallest beam radius of $5 \mathrm{~mm}$ at the central mirror this corresponds to approximately $150 \mathrm{~kW}$ circulating power or a power buildup of the $\mathrm{PC}$ of $P B_{\mathrm{PC}} \approx 5000$. The $\mathrm{RC}$ will not suffer from too high intensities on the mirrors such that its power buildup will be limited by the optical losses. If we take a typical value of $8 \mathrm{ppm}$ losses per mirror and allow for an equal amount of roundtrip clipping loss the power buildup in an impedance matched cavity will be $P B \approx 40000$. Based on the above arguments we have chosen $P B_{\mathrm{PC}} \approx 5000$ and $P B_{\mathrm{RC}} \approx 5000$ as the ALPS-II cavity design parameters.

The eigenmode in the RC has to be an extension of the PC eigenmode to match the Gaussian beam parameters of the regenerated field. It can be shown that the Gaussian beam with the smallest clipping losses for a given constant magnet aperture and identical length of the PC and RC is the one with the beam waist in the center between the two cavities and a Rayleigh range equal to the length of a single cavity. For such a beam the aperture radius required to keep the clipping losses small enough to achieve the power buildup goals is plotted over the cavity length Fig. Q 9 .

Under the assumption that the HERA magnets can be straightened to give an aperture of $d=$ $50 \mathrm{~mm}$ - with the free aperture taken to be $10 \mathrm{~mm}$ smaller, to account for alignment tolerances, the waviness of the vacuum pipe, and fluctuations of the laser position - a cavity length of $100 \mathrm{~m}$ is possible.

In principle the PC could be longer as larger clipping losses are acceptable in this cavity. Given that the total length is limited by the infrastructure, we have, however, not included this option to allow for mirrors with identical radius of curvature (ROC) in both cavities.

A key component of the ALPS-II optical design is the central breadboard. This breadboard is marked with a beige-colored background in the optical layout shown in Fig. 12, a picture of the breadboard during setup of the $1 \mathrm{~m}$ tabletop experiment can be seen in Fig. 10. It provides a long 


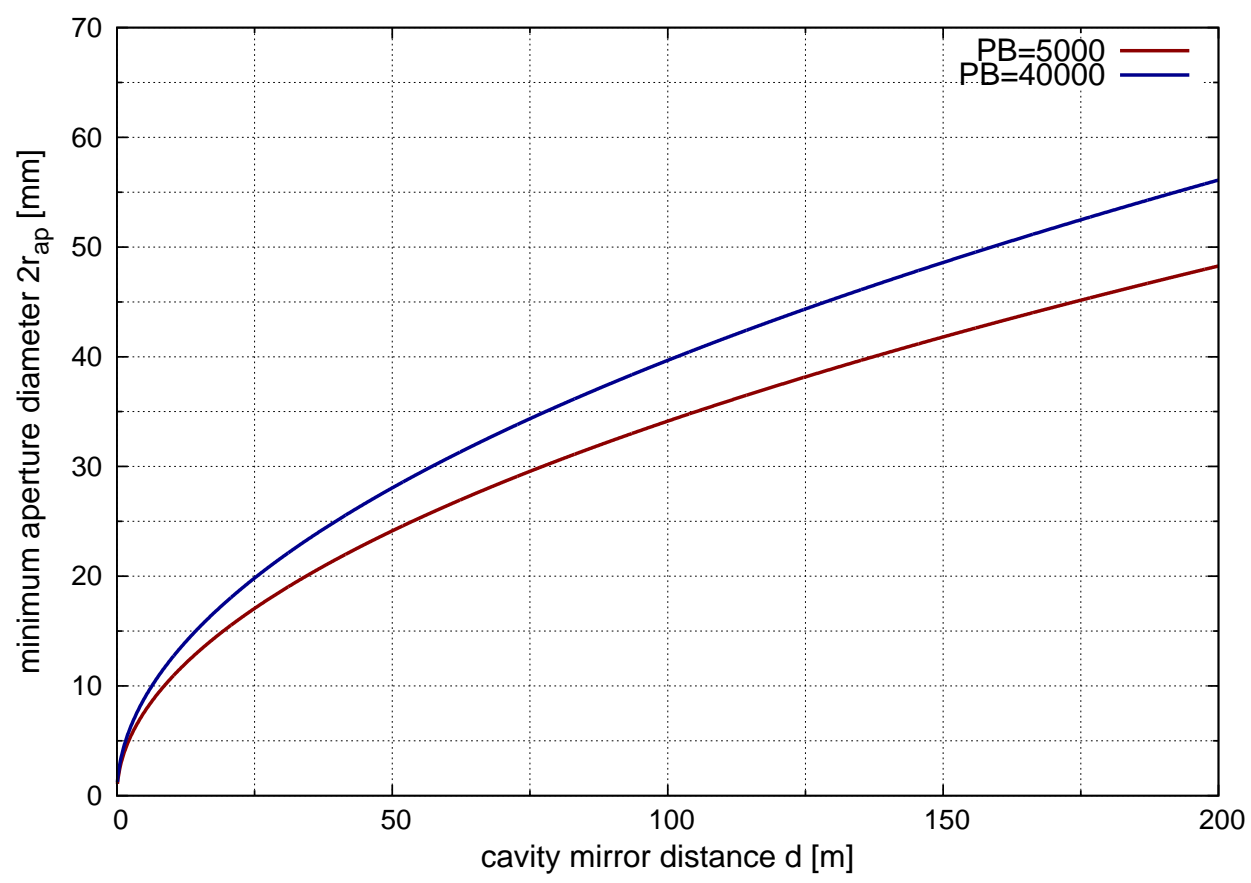

Figure 9. Minimum aperture diameters $2 \cdot r_{a p}$ required to allow for a power buildup of 5000 for the PC (red, lower line) and 40000 for the RC (blue, upper line) plotted over the cavity mirror distance $d$. Additional mirror losses of $8 \mathrm{ppm}$ are assumed for each cavity mirror.

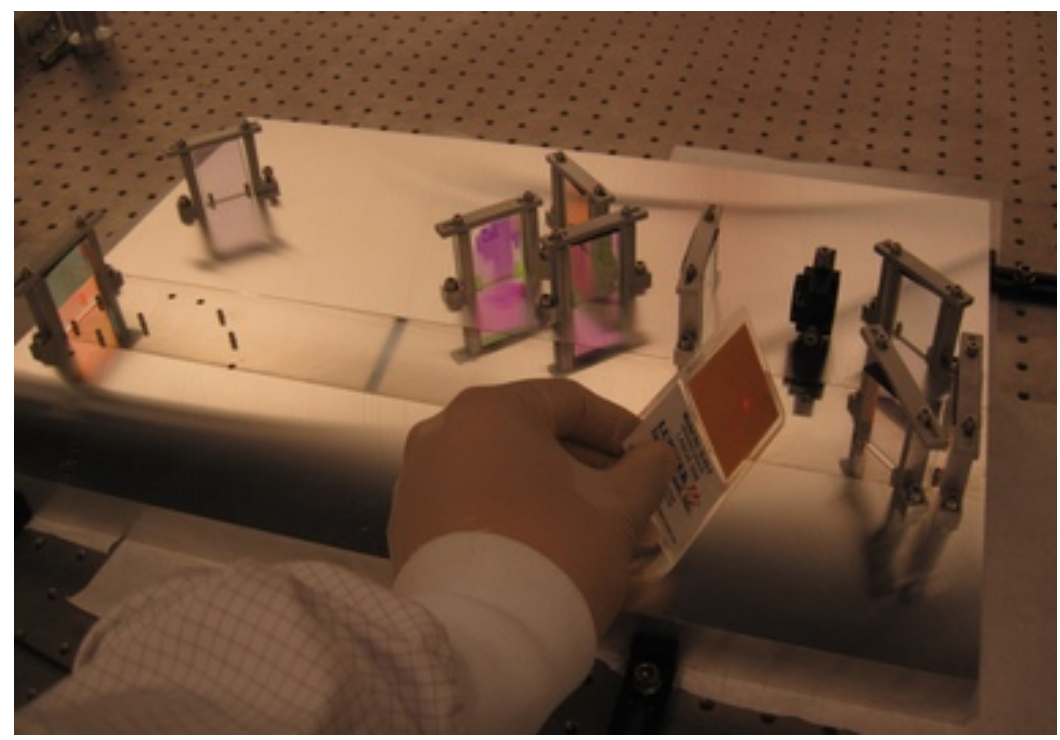

Figure 10. Picture of a prototype of the ALPS-II central breadboard during setup of the $1 \mathrm{~m}$ tabletop experiment at AEI. 
term stable platform for mounting the central cavity mirrors and the quadrant photodiodes used as alignment references.

The mirror labeled as CBS1a is the end mirror of the PC and is chosen to be flat $(\mathrm{ROC}=\infty)$. This choice will force the optical axis of the PC to be perpendicular to the surface of CBS1a. A fraction of $90 \%$ of the beam transmitted by CBS1a is directed towards mirror M2. Mirror M2 transmits $1 \%$ of the beam which is detected by the quadrant photodiode QPD3. A control loop shown in Fig. 12 is used to steer the input mirror PIC of the PC such that the beam on QPD3 hits a reference point. Once this control loop is operating, all degrees of freedom of the PC axis are fixed with respect to the central breadboard.

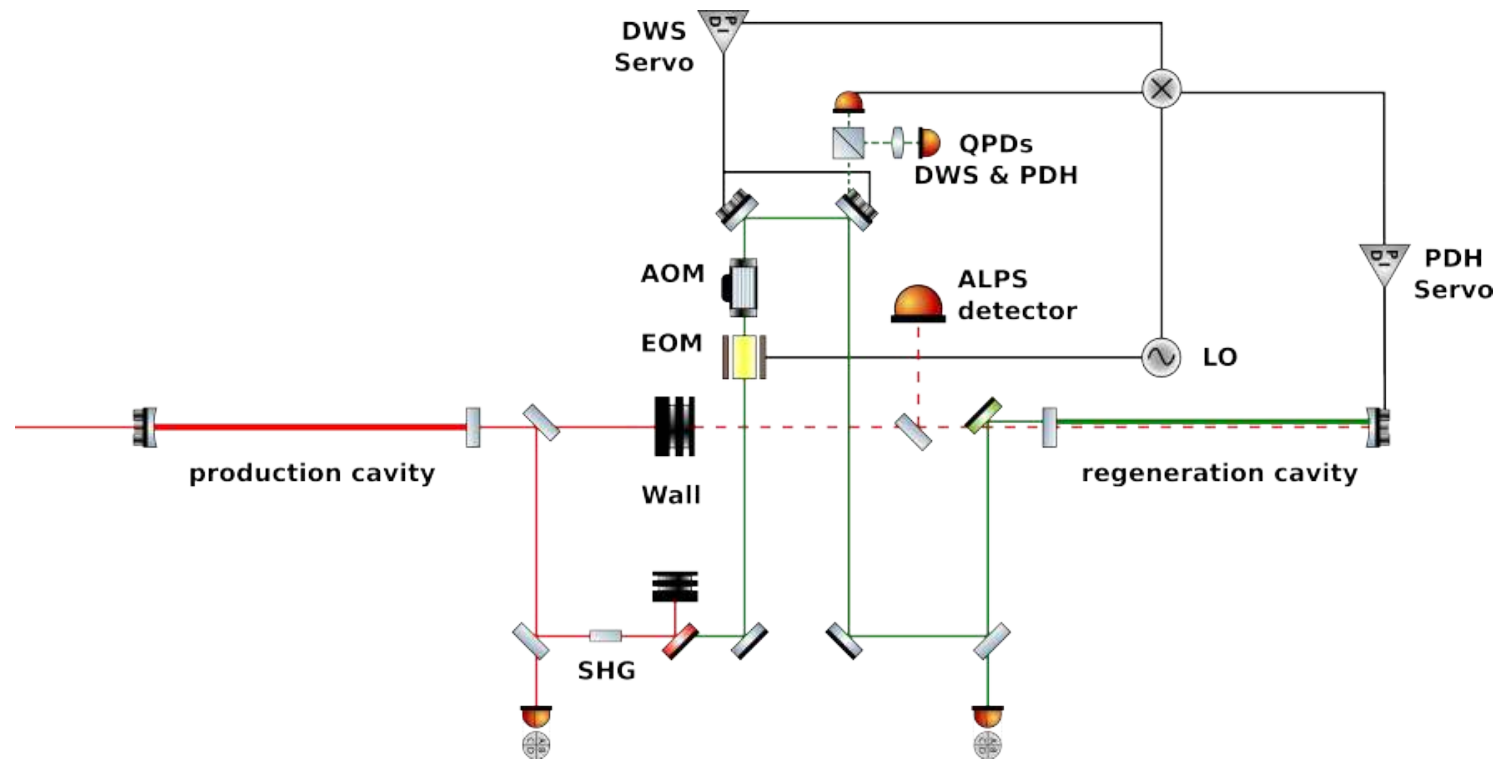

Figure 11. Schematic of the ALPS-II regeneration cavity including control loops.

The main fraction of the beam is reflected by M2 and send into a KTP non-linear crystal. A beam with a wavelength of $532 \mathrm{~nm}$ is produced via the second harmonic generation (SHG) process. A dichroitic mirror separates the green and red beam and directs the red beam to a beam dump. A fraction of $90 \%$ of the green beam will be directed towards an optical setup not mounted on the central breadboard (and outside of the vacuum tank which houses the central breadboard, see Fig. (12). In this setup the green beam is frequency shifted via an acousto-optic modulator $(\mathrm{AOM})$ and phase modulation sidebands are added to the beam for the PDH locking of the RC. The conditioned green beam is now send back to the central breadboard and passes the "wall" through a set of dichroitic mirrors to make sure that no $1064 \mathrm{~nm}$ light enters the RC and detection area. After the "wall penetration" the green beam is injected into the RC. The RC cavity consist of the flat mirror CBS2a and the curved mirror REM which can be controlled in the direction of the cavity axis for cavity length control and in rotation and tilt for cavity alignment control. The mirrors of the $\mathrm{RC}$ are coated to form a resonant cavity for $1064 \mathrm{~nm}$ with a power buildup (PB) of 40000 and for $532 \mathrm{~nm}$ with a PB of approximately 60 . The green beam reflected by the RC traces back the path of the incoming beam to the quadrant photodiodes QPD 5 and QPD 6 which are used as sensors in 


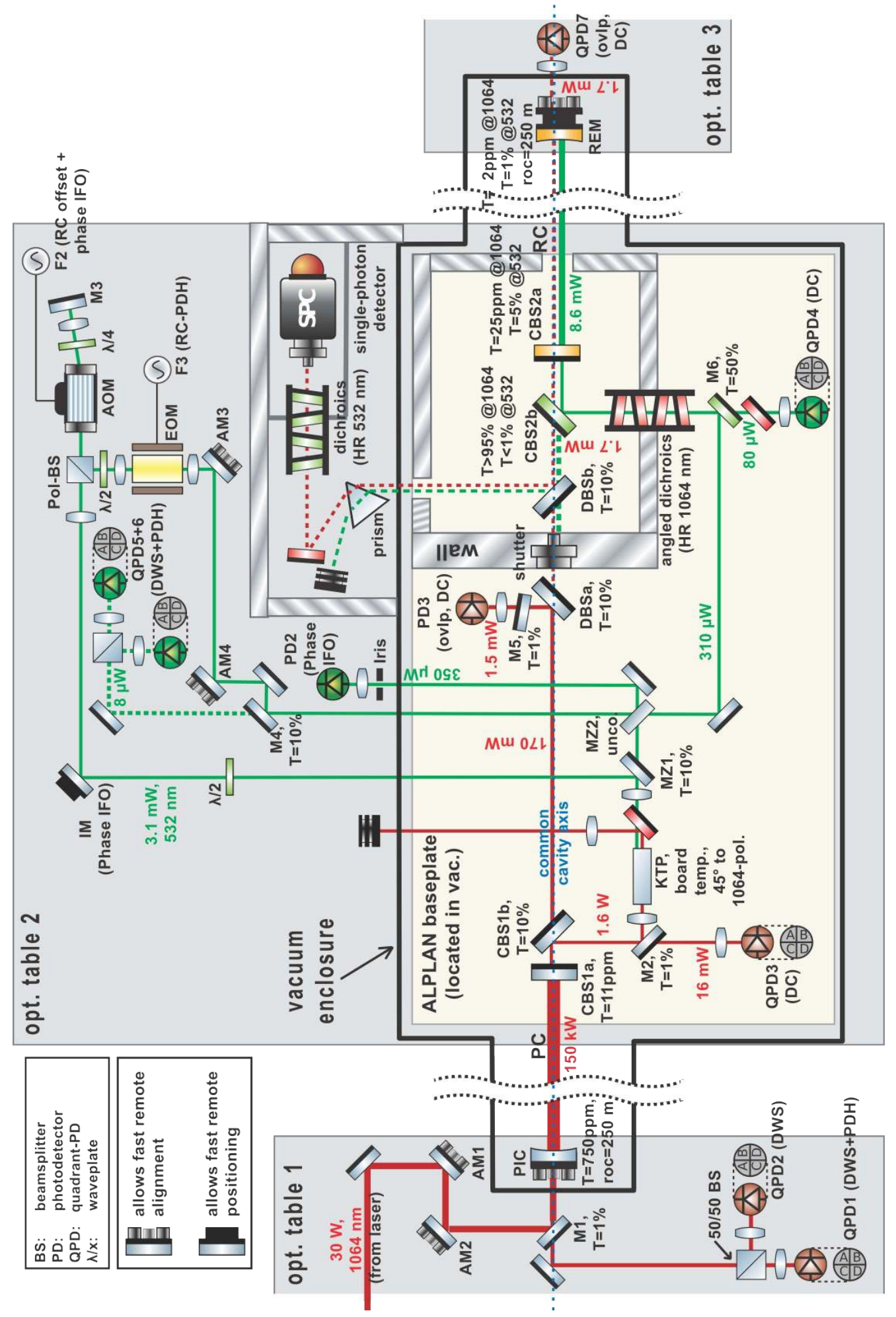

Figure 12. Layout of the ALPS-IIb and ALPS-IIc optical tables.

differential wavefront sensing (DWS) and PDH sensing schemes to generate error signals for the 
alignment control of the green beam with respect to the cavity eigenmode and the length of the PC with respect to the resonance of the green beam. Control loops feeding back to the steering mirrors AM3 and AM4 will keep the green beam aligned to the cavity eigenmode and feed-back to the cavity end mirror REM will change the cavity length to make it resonant for the green beam. A schematic of the control loop layout can be seen in Fig. [11.

The remaining task is to make the eigenmode of the RC co-linear with the eigenmode of the PC. For this we have to mount the two flat central mirrors of these cavities in parallel and rigidly on the breadboard (see below for required precision). This ensures that the cavity axis are parallel. Once the red and green beams are resonant in the respective cavity we can open the shutter in the wall to allow a $1064 \mathrm{~nm}$ beam to impinge on the RC. By rotating and tilting mirror REM we can laterally shift the RC eigenmode until it matches the incoming $1064 \mathrm{~nm}$ beam. During this process we might need to change the frequency offset between the green and red beam via the AOM to meet the resonance condition for the green and red beam at the same time. Once the $1064 \mathrm{~nm}$ beam resonates in the RC we note the position of the green beam on QPD4 as the spatial reference for the RC eigenmode. This reference position and the normal to the surface of mirror CBS2a fully define the RC axis. Hence a control loop feeding back to tilt and rotation of REM to keep the beam on this reference position fixes the location of the RC eigenmode relative to the central board and co-linear with the PC axis. If the positions of CBS1a, QPD3, QPD4 and CBS2a do not move with respect to each other and all control loops are closed the $1064 \mathrm{~nm}$ beam will be resonant in the $\mathrm{RC}$ as will be any light field regenerated from WISP particles. A typical measurement sequence would involve an open central shutter at the beginning and end of the measurement (and possibly in between) to ensure alignment and resonance conditions are right and a closed central shutter for the WISP search. During those searches possibly regenerated $1064 \mathrm{~nm}$ photons will be transmitted by CBS2a and CBS2b and steered by DBSb to the main ALPS detector (CCD or TES). It is essential that none of the $532 \mathrm{~nm}$ photons used to control the RC is accidentally detected and interpreted as regenerated $1064 \mathrm{~nm}$ photons. Hence the $532 \mathrm{~nm}$ photons have to be reflected, absorbed or spatially split from the $1064 \mathrm{~nm}$ beam path after leaving the RC.

Dedicated studies to determine the production probability of $1064 \mathrm{~nm}$ photons (for example by fluorescence effects) from the $532 \mathrm{~nm}$ light used to lock RC are under way. First results have shown that this probability is below $10^{-17}$. We plan to set up further experiments to mimic the optics of the regeneration cavity in more detail and to exploit polarization effects to discriminate between fluorescence photons and light from reconverted WISPs. For ALPS-II, a production probability for $1064 \mathrm{~nm}$ from $532 \mathrm{~nm}$ photons of less than $10^{-21}$ photons is to be achieved.

Once the shutter in the wall is closed, we rely on the stability of all components on the central breadboard. As the green beam used for the RC control leaves the breadboard and the vacuum system it might be subject to phase fluctuations caused by air turbulences and/or vibrations of the optical components outside of the vacuum system. Hence we will set up a Mach-Zehnder interferometer with the beam splitters MZ1 and MZ2. The output port of this interferometer is sensed by PD2 and will give us information about the phase fluctuations. If required, a control loop feeding back to mirror IM will be used to correct for these fluctuations.

\subsubsection{Expected performance}

We expect that we can reach a light power level of $150 \mathrm{~kW}$ traveling into the direction of the "wall" 
and to enhance the detection efficiency of regenerated photons by an RC with a power buildup of 40000. Several requirements have to be fulfilled to achieve this performance:

1. The total lateral and angular beam shift introduced to the $1064 \mathrm{~nm}$ beam by optical components between the two cavities on the central breadboard has to be smaller than $1 \mathrm{~mm}$ and $10 \mu \mathrm{rad}$, respectively, because any beam shift is not seen by the particles transversing the wall and hence reproduced light would not match the RC eigenmode.

2. To allow parallel alignment of the optical cavities, the central mirrors have to be parallel to within $10 \mu \mathrm{rad}$.

3. Drifts (e.g., caused by thermal gradients) of the components on the central board have to be small enough to meet the pointing requirements given in 1 . The mismatch between twice the red resonance frequency and the green resonance frequency of the RC has to be stable to a fraction of 0.2 of the RC linewidth (corresponding to approximately $95 \%$ of the maximum power buildup).

4. The actuator range of the different control loops has to be large enough to compensate the free running peak-to-peak fluctuations of the relevant degrees-of-freedom.

5. The control-loop disturbance reduction has to be large enough to reduce the deviation of the cavities from their operation points such that the power buildup factor can be kept at a value larger the 0.95 times the maximal buildup.

Simulations of the optical setup and estimations of the control loop performance indicate that all the requirements can be met with state-of-the-art optics, electronics and fabrication processes.

\subsection{Cleanroom design}

\subsubsection{General considerations}

The setup of the optical components of the experiment has to fulfill the following requirements:

1. The optical components outside and inside the vacuum must be kept free of dust particles after being cleaned, during the mounting process and during the experimental runs. Due to the high cavity power buildup aimed for, the optical components in the experiment, especially the cavity end mirrors, are most sensitive to dust particle contamination. Even a degradation of a few ppm in the reflectivity of an end mirror would lower the performance of the regeneration cavity intolerably.

2. Mechanically and thermally stable conditions have to be maintained during the operation of the experiment. Only minimal position changes of optical components are tolerable due to the limited expansion range of the piezo-electrically driven mirror holders. A movement above a few $\mu \mathrm{m}$ in beam direction of an end mirror can not be compensated by the feedback loop for cavity length stabilization.

3. For the operation of the infrared laser (continuous-wave output: $35 \mathrm{~W}$ at $1064 \mathrm{~nm}$ ) a safe working environment has to be created and maintained at all times, with special attention to the setup phase of the experiment. 


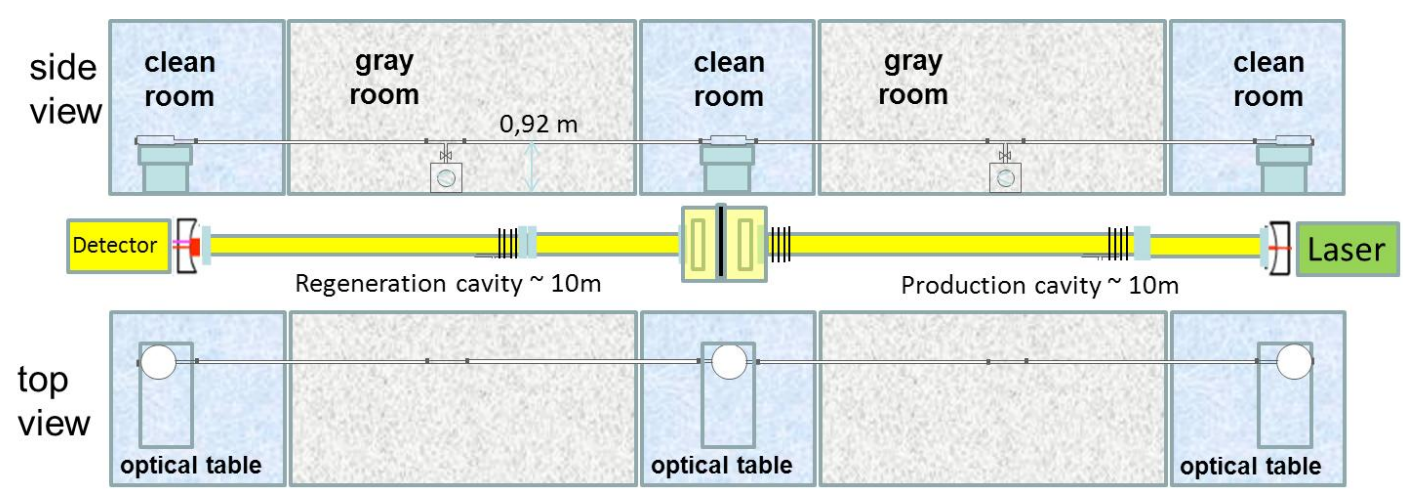

Figure 13. Experimental setup for ALPS-IIa in building 50 room 607.

\subsubsection{The cleanrooms}

The laboratory space for ALPS-IIa including three cleanrooms has been set up in 2011 already. The layout of the cleanrooms for the ALPS-IIb and ALPS-IIc experimental setups is based on the approach used for ALPS-IIa in the laser laboratory housed presently at DESY in building 50 (see Fig. (13). There are three cleanrooms containing:

1. the laser and the vacuum chamber with the central breadboard,

2. the vacuum chamber with the second end mirror of the production cavity and the first end mirror of the regeneration cavity and

3. the vacuum chamber with the second end mirror of the regeneration cavity.

Adjacent to the cleanrooms are gray-rooms which serve as airlock to the outside. Cleanroom conditions will be ensured applying the following measures:

1. The floor inside the cleanrooms and the gray-rooms is regularly cleaned.

2. In each gray-room entrance area adhesive carpeting is used to avoid propagating dirt.

3. Persons use cleanroom suitable clothing and foot wear. To enter a gray-room an additional cleaning lock space has to be passed, to reach the required cleanliness.

4. A flow of $200 \mathrm{~m}^{3} / \mathrm{h}$ of fresh air is provided, sufficient for up to 4 persons. In order to refresh breathing air, filtered air (H12) from the outside is blown into each gray-room. The resulting turbulent flow of mixed air also reduces the gray-room dust particle concentration compared to the outside.

5. To reduce dust particle concentration in the cleanroom, clean filtered air (H14) from the adjacent gray-room is blown in turbulently. The air exits the cleanroom back into the grayroom. Every two minutes the air volume in the cleanroom is mixed with an equal volume of filtered air. In that way the dust particle concentration in the gray-room is also reduced and the breathing air in the cleanroom is continuously refreshed. 
6. The enclosure of the laser table in the cleanroom has a roof with vertical transparent blinds. Within the enclosure a separate filter unit produces a clean horizontal laminar displacement flow across the laser table, which is always directed against the person reaching through the blinds and performing manual work. Dust particles from the person's moving hand or arm will not move down towards the optical components on the laser table, but instead leave the enclosure through the blinds.

Dust particle concentrations are expected to be the same as in the ALPS-IIa laboratory (down to $0.3 \mu \mathrm{m}$ size):

- 100000 particles $/ \mathrm{ft}^{3}$ outside,

- 5000 particles $/ \mathrm{ft}^{3}$ in the gray-room,

- 200 particles $/ \mathrm{ft}^{3}$ in the cleanroom and

- 0 - 1 particles/ $\mathrm{ft}^{3}$ within the laser table enclosure in front of a working person's hand reaching in.

These numbers match the requirements. About two hours after the last person has left a cleanroom of ALPS-IIa, no dust particles are measured anymore by a particle counter located in the cleanroom outside the enclosure. For more effective dust decontamination of clothing, the use of an ionization gun will be tested in the ALPS-IIa laboratory.

At present it is planned to support the optical tables in the tunnel cleanrooms from the top of the HERA tunnel, as there is little space on the floor due to big pipes for cooling water and Helium gas (see Fig. 34). This concept will be tested at the setup of ALPS-IIb. The cleanroom between the two magnet strings will be located in a HERA hall and the optical table will be supported from below.

Like in the ALPS-IIa setup the breadboards containing the optical elements will be supported from the optical tables, decoupled mechanically from the vacuum vessels, see Fig. 36, which are supported from the floor of the cleanrooms.

For ALPS-IIc the HERA kicker-bypass will be installed allowing the magnet current and the cryogenics to bypass the optical setup in the middle of the experiment. To compensate the force of about $80 \mathrm{kN}$ by the atmospheric pressure on the end flanges of the kicker-bypass, the endflanges are connected by 3 tension rods. To allow access to the optical elements in the vacuum vessel in the cleanroom, the tension rods have to be replaced by a steel girder construction. The detailed design for the construction of the clean/gray rooms remains to be done. It is assumed that the construction will be done in a similar way as for ALPS-IIa applying a drywall installation.

\section{Temperature Stability}

As for the setup of ALPS-IIa, the air blown into the cleanroom will also be used for cleanroom temperature stabilization by mixing the input flow with the output from an air conditioner unit. The temperature sensor of a feedback loop will be placed above the laser table. The same feedback system was used in the ALPS-I laser laboratory where a long term stability of better than $\pm 0.1{ }^{\circ} \mathrm{C}$ was reached. The three air conditioning units will use cold water from the $8{ }^{\circ} \mathrm{C}$ DESY cooling water system. 


\section{Laser Safety}

As for the ALPS-IIa laboratory, a safe laser environment will be set up in accordance with the required safety rules (DIN/EN 60825). An interlock system based on programmable logic controllers is foreseen which closes the exit shutter of the laser if any door connecting a cleanroom to its adjacent gray-room, and the gray-room exit door are open at the same time.

\subsection{Detection system}

The detection of the possible regenerated photons at ALPS is very challenging, mainly due to their expected low energy and low rate. The wavelength of the photons at ALPS-II being $1064 \mathrm{~nm}$ reduces the detection options and makes silicon-based detectors less efficient due to the proximity of the energy of the Si band gap. Caused by the low rates, the most important requirement of a detector system for ALPS-II is extremely low dark count rate (at least less than $10^{-3} \mathrm{~s}^{-1}$ ), which should be coupled with a high detection efficiency.

For ALPS-II two detection options are pursued:

- a PIXIS 1024B CCD camera and

- a Transition-Edge Sensor (TES).

Whereas the CCD camera has already been used for ALPS-I and only needs to be calibrated for the ALPS-II requirements, an ALPS-II-specific TES set-up is currently being newly developed by the ALPS collaboration.

\subsubsection{Technical challenges}

The main requirements for the detection of the low rates of single photons with a wavelength of $1064 \mathrm{~nm}$ at ALPS-II are (in the order of their importance):

1. low dark count and background rate,

2. high efficiency,

3. long-term stability,

4. good energy resolution and

5. good time resolution.

The CCD camera is proven to be a viable option and has the advantage of being ready-to-use. It satisfies the first three criteria, but does not excel in them. The dark current of the CCD is of the order of $10^{-3}$ electrons per pixel per second and its efficiency at the ALPS-II wavelength is about $1.2 \%$ (further details see Sect. 3.4.3).

Transition-edge sensors [89] exploit the rapid change of the resistance at the superconducting phase transition. This enables them to reach high sensitivity [90] and achieve highest quantum efficiencies [91]. In addition, good energy and timing resolutions are possible. In comparison to a Si-CCD, the quantum efficiency of a TES at $1064 \mathrm{~nm}$ is larger by nearly two orders of magnitude and additionally the dark count rate is lowered by a considerable factor. The additional benefit of 
a single-photon spectroscopy opens up attractive avenues to additionally reduce the background through cuts on the pulse-height. The timing allows for precise definition of good-time-intervals when the cavity is locked. Hence, an unstable operation of the cavity poses no problem for the TES detector. However, building and operating a TES setup is more challenging than to use a CCD camera. Points of major importance for the success are the control of the environment (e.g., magnetic field etc.) at $\mathrm{mK}$ temperature, and the sufficient suppression of different possible backgrounds. In the field of experimental searches for rare events (e.g., direct Dark Matter experiments), TES detectors have been operated stable over the course of months (see e.g., CRESST II [92]).

\subsubsection{Conceptual design}

\section{CCD camera}

The design of the CCD detector is based upon the experience from the ALPS-I experiment. The beam of regenerated photons is focused with a lens onto the sensor area of a CCD camera. To achieve the best performance, the size of the focal spot should be minimal (in the ideal case one pixel).

The CCD camera in ALPS-II is the same that was used in the ALPS-I experiment, a PIXIS $1024 B$ by Princeton Instruments. The chip is a back-illuminated e2v CCD47-10. It has $1024 \times 1024$ $13 \times 13 \mu \mathrm{m}$ sized pixels. The chip can be cooled to a minimum of $-70{ }^{\circ} \mathrm{C}$. The read-out electronics allows for two read-out speeds of $2 \mathrm{MHz}$ and $100 \mathrm{kHz}$ with associated read-out noise of $13.73 e$ and $4.09 e$, respectively. It is capable of binning the pixels into logical pixels, thus reducing the impact of the read-out noise if the signal cannot be focused on one pixel.

Neighboring pixels can be used to veto cosmic rays and ambient radioactivity.

To optimize the focal spot, the shutter of the central breadboard will be opened so that infrared light will enter the regeneration cavity. This light will follow exactly the path of regenerated photons. Hence, its image can be used to optimize the focal spot of the regenerated photons. Because of the high intensity of this light $(1.7 \mathrm{~mW})$, a dedicated filter will be installed in the beam to protect the camera, which does not change the position of the image. This will be verified by rotating the filter around the beam-axis.

\section{TES detector system}

The working principle of a TES is briefly explained in Fig. 114. The TES is a microcalorimeter measuring the temperature difference $\Delta \mathrm{T}$ of the absorber material through the rapid change of the resistance at the superconducting phase transition, which is proportional to the temperature increase at the set point. In the case of ALPS, and in general for optical detection, the TES detector serves as absorber and thermistor at the same time.

The research and development of TES for detection of optical/infra-red photons is actively carried out at metrology institutes like NIST (in the U.S.), AIST (in Japan), INRIM (in Italy) and PTB (in Germany). The ALPS collaboration has established contacts to these institutes and is collaborating regarding the setup of the ALPS TES detector system and the TES chips (see below).

In 2011, first experience was gathered through an extensive collaboration with the Universities of Trieste and Camerino, building up a TES detector with a dilution refrigerator. For a TES setup essentially four components are required: the TES detector, a SQUID-current sensor, a cooling 


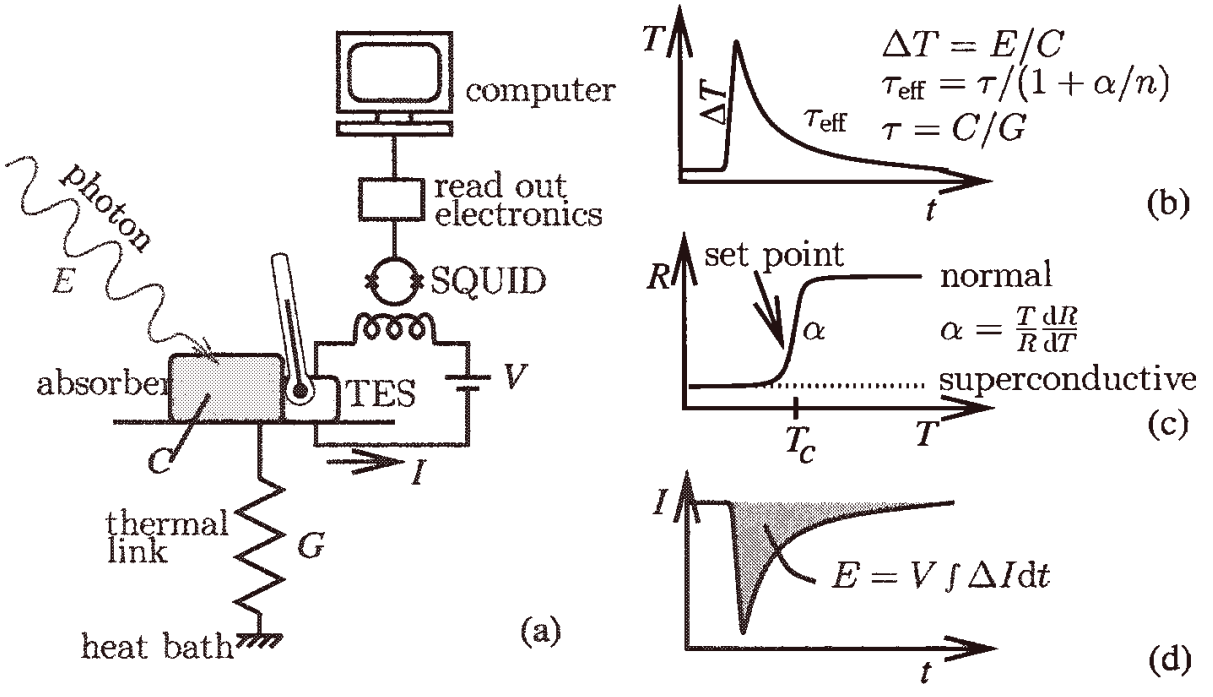

Figure 14. Working principle of a TES. (a) A photon sensitive absorber with heat capacity $C$ is connected to the TES thermistor. The absorber is coupled with a weak heat conductivity $G$ to a "heat" bath. The TES is voltage-biased and the current of the bias circuit is read-out by a SQUID connected to read-out electronics. (b) The absorption of a photon with energy $E$ causes a rise in temperature. The TES cools down again and is back to its set point after the time $\tau_{\text {eff. }}$. (c) As the TES is operating in the superconducting transition region, there is a change of electrical resistance $R(T)$. (d) The current of the circuit changes, which can be readout by the SQUID, which is magnetically coupled to the circuit. Integrating over the current signal $I(t)$ leads to the incident photon energy. Figure from [93].

system to reach and maintain a sufficiently low temperature, and the optics to feed the light signal to the sensor. The realization and the status of the TES detector of ALPS-II is split into these four fields, which are described in the following and summarized in Fig. 15.

Sensor: TES Chip TES detector chips are not available commercially. Research groups either manufacture their own detectors or obtain detectors by collaborating with metrology institutes, most notably NIST and AIST. NIST and AIST have developed highly efficient multilayer TES optimized for various optical and near-infrared wavelengths. ${ }^{11}$

The two following TES would be suitable for the ALPS-II wavelength of $1064 \mathrm{~nm}$ :

- NIST TES with optical structures to couple light and metallic mirrors reaching $98 \%$ quantum efficiency for $1064 \mathrm{~nm}$ [94]

- AIST TES with optical structures to couple light and dielectric mirrors reaching $98 \%$ quantum efficiency for $850 \mathrm{~nm}$ [95]

These TES have low critical temperatures $\left(\mathrm{T}_{c}\right)$ to reduce the thermal fluctuation noise and to make the device more sensitive. The NIST TES use Tungsten (W) as sensor material and become superconductive between 100 and $200 \mathrm{mK}$, the AIST TES are made of Titanium/Gold (Ti/Au) leading to a higher critical temperature of around $300 \mathrm{mK}$.

\footnotetext{
${ }^{11}$ Both institutes have concentrated on the telecommunication wavelengths 1310 and $1550 \mathrm{~nm}$.
} 

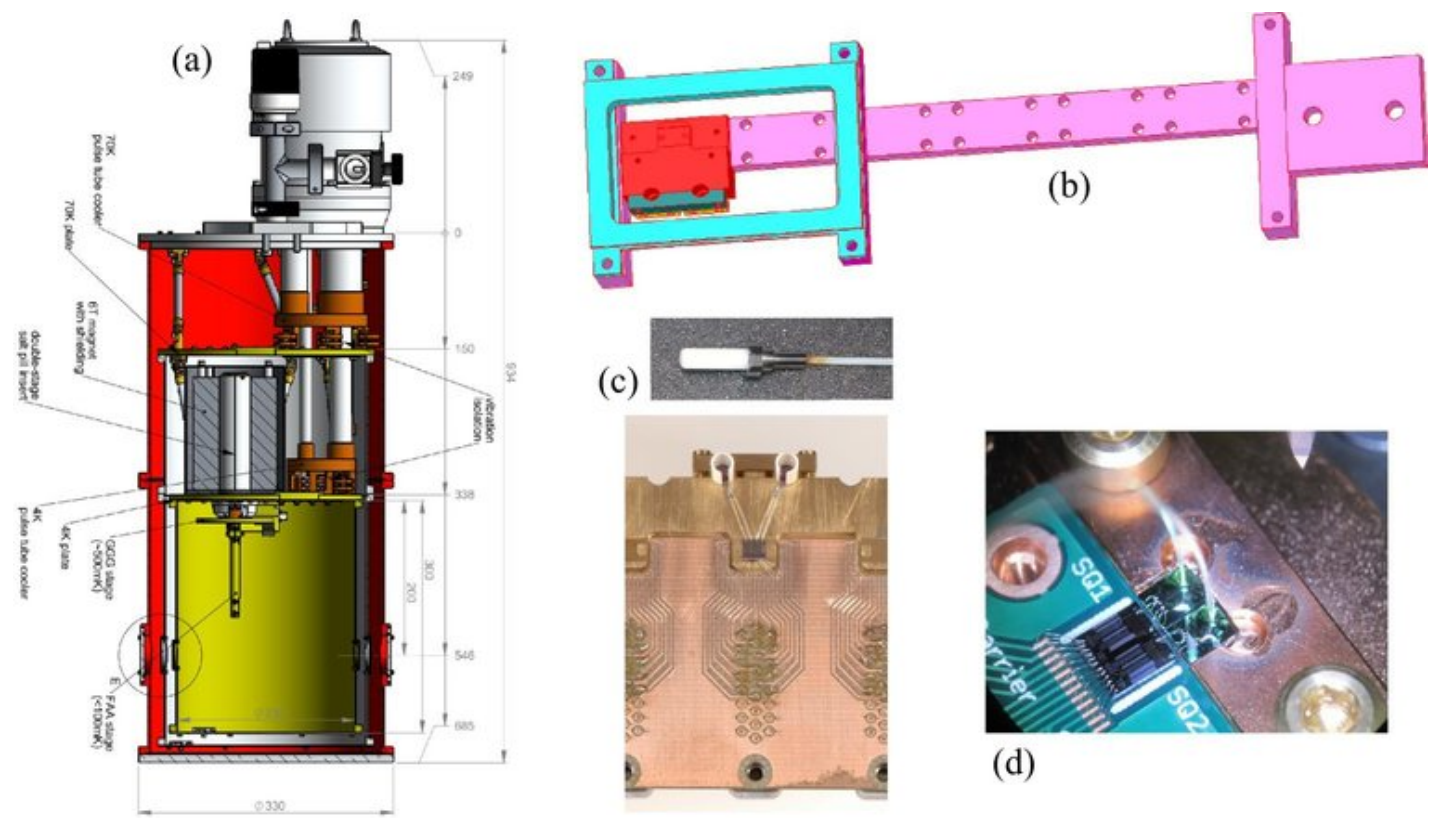

(c)
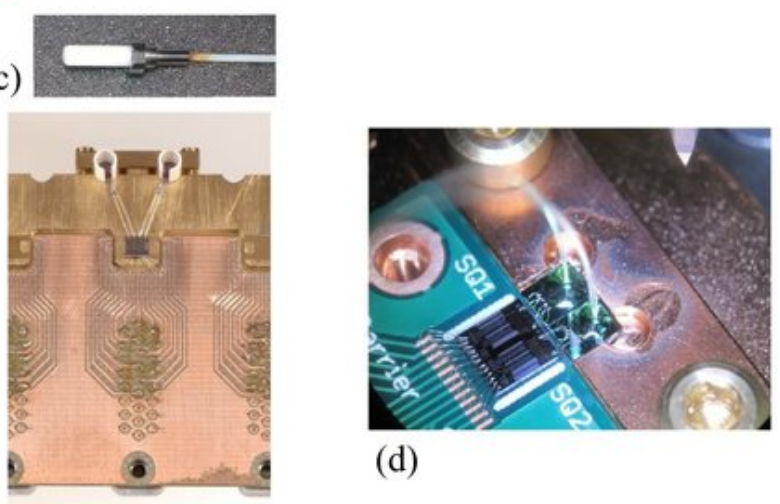

(d)

Figure 15. Collection of components of the ALPS TES detector system: (a) Sketch of the ADR: In the upper part there is a two-stage pulse-tube cooler connected to the $70 \mathrm{~K}$ and $4 \mathrm{~K}$ stage. The dashed gray part in the middle indicates the salt pill with $6 \mathrm{~T}$ magnet for ADR cooling. The light green part is the mK-environment with the cold finger. (b) Sketch of the detector bench attached to the cold finger: The detector bench, made of copper, holds the sensor module (red), where one SQUID and two TES chips are located. The sensor module is surrounded by a brass part (light turquoise) for a magnetic field coil to compensate background magnetic fields. (c) Pictures of fiber end and sensor bench: In the bottom picture a sensor module for NIST TES and PTB SQUID chips is displayed. The two white ceramic ferrules surround the TES chips and can hold the fiber end pictured above. (d) Picture of TES/SQUID design by AIST: Two fibers are glued to TES chips, which are connected to the SQUID located on a circuit board. [Pictures from (a) Entropy, (b) DESY, (c) NIST/PTB, (d) AIST]

ALPS is in contact with NIST and AIST and will receive chips from both institutions in order to measure their performance for $1064 \mathrm{~nm}$. It is planned to optimize the chips [96] achieving a low-noise high-efficient TES detector for single $1064 \mathrm{~nm}$ photons by using

- dielectric mirrors (narrowband ${ }^{12}$ ) instead of metallic mirrors (broadband) and

- an optimized multilayer structure for coupling light with $1064 \mathrm{~nm}$,

if that proves to be beneficial for the ALPS-II experiment.

Read-out: SQUID-Current Sensor TES are mostly read-out by low- $T_{c}$ dc SQUID current sensors, consisting of the SQUID itself and a read out electronic. These sensors are current to voltage transformers (transimpedance elements): To give some numbers, a $1 \mathrm{eV}$ energy input would cause a current of $\mathscr{O}(100 \mu \mathrm{A})$ in the TES circuit (see Fig. 15), which results in a voltage pulse in the range of 1 to $100 \mathrm{mV}$. Low- $T_{c}$ SQUIDs can be operated at mK-temperatures.

The SQUID sensors for the ALPS setup will be supplied from the PTB ${ }^{13}$ in Berlin. PTB

\footnotetext{
${ }^{12} \mathrm{~A}$ narrowband detector could reduce a broadband background.

${ }^{13}$ Physikalisch-Technische Bundesanstalt, Working Group 7.21: Cryosensors
} 
SQUIDs are already in use with NIST and AIST TESs, so the PTB group is joining a collaborative effort with ALPS to find out which SQUID-TES design is the most robust one for the ALPSpurpose and, more generally, to test TES applications. This will allow ALPS to profit from the great expertise of PTB regarding SQUIDs.

ALPS has already purchased a TES/SQUID read-out electronic from Magnicon: Two channels are for SQUID read-out allowing the operation of two TES sensors in parallel for cross checks. Additionally, a compensating magnetic field coil can be operated to prevent degradation of performance of SQUID, when operated in an ADR.

Optics: Fiber Feed-in To guide the light signal into the cryostat and finally onto the TES an optical fiber is the method of choice. The anticipated design aims at an optimized optical efficiency and will have to address the following issues:

1. fiber-to-TES coupling in the cold

2. fiber vacuum feed-through as interface between free space and cryogenic environment

3. free-optic-to-fiber coupling

The fiber-to-TES coupling will be done with specific methods developed at NIST and AIST. NIST uses a modified standard fiber connector, whereas AIST uses UV curable resin for gluing the fiber end to the TES chip. With both approaches low-loss couplings and a detection efficiency up to $98 \%$ were reached [97, 95].

For a low-loss working fiber vacuum feed-through with a continuous fiber a standard Swagelok with a Teflon ferrule will be used, following [98]. DESY's workshop has already built a single-fiber feed-through, a feed-through for two fibers is currently under construction.

Most importantly, the incoming signal photons need to be coupled into the fiber. The beam diameter at the coupling mirror of the regeneration cavity (CBS2a in Fig. 12) will be $11.6 \mathrm{~mm}$. This will contain $86.5 \%$ of the total power (i.e., $2 \sigma$ of the Gaussian beam profile). Thus, to capture about $98.9 \%$ of the total power, an optic is suitable that collimates a $17.5 \mathrm{~mm}$ diameter beam into a single mode fiber with about $5 \mu \mathrm{m}$ diameter. First tests and experience in the ALPS collaboration and from other groups demonstrated that with a simple collimator lens a coupling efficiency about 60 to $80 \%$ is easily reachable due to coupling losses.

For reducing the background there are different approaches considered to filter out other wavelengths. The fiber itself can be used as a simple filter by using a HI1060 fiber with a operating range of 980 to $1180 \mathrm{~nm}$ instead of the standard fiber SMA980 with a operating range from 970 to $1650 \mathrm{~nm}$. Other possible options are reflecting coatings at the cold end of the fiber and filters (including polarization) within a fiber circulator design. These options will be further pursued, if the background level is too high.

Cryogenic System: ADR An ADR was found a suitable solution for the requirements of the TES setup in a milli-Kelvin environment. The University of Hamburg as collaborator of ALPS ordered in November 2011 an ADR from the company Entropy. It was delivered in late July 2012, tested at the PTB in Berlin (see Fig. 16) and is now operational at DESY in Hamburg.

The ADR is a no-liquid-cryogens cryostat with a closed pre-cooling cycle: Integrated is a twostage pulse-tube cooler with which the cooling stages of $70 \mathrm{~K}$ and $4 \mathrm{~K}$ are reached. Then using a 
6 T magnet and a double-stage salt pill unit the milli-Kelvin environment is established through the principle of adiabatic demagnetization.

A cryogen-free cool-down is possible within $20 \mathrm{~h}$. The hold time at $100 \mathrm{mK}$ is about $48 \mathrm{~h}$ depending on the cold mass. The recharge time to re-establish the mK-environment is about $30 \mathrm{~min}$ according to the datasheet [99]. With the ADR the aim of a compact and robust, transportable system with a quick cool-down and easy handling is in reach.

The following components for the setup of the TES inside the ADR are currently being designed and built:

- detector bench attached to the ADR cold finger for TES and SQUID chips including coils compensating for magnetic field

- cryo-cable for read-out from vacuum feed-through to $4 \mathrm{~K}$ stage

- circuit board at $4 \mathrm{~K}$ stage

- vacuum-feed-through for optical fibers

- thermal anchoring of cables and fibers coming from $300 \mathrm{~K}$

- shields against thermal and magnetic/electric radiation (currently several solutions being designed, the final choice will depend on the actually measured background levels)

After assembling the ADR and characterization of the completed TES detector the complete detector system will be moved to Hamburg and set up at the ALPS site. The ALPS site is fully equipped to operate the ADR with $300 \mathrm{~V}$ supply and purified water for the compressor ${ }^{14}$ of the pulse-tube cooler.

As a status some pictures of the ADR system at PTB are shown in Fig. 16. The ALPS collaboration will soon reach mK-regions, after the adjustment and fine tuning of the heat switch.

\subsubsection{Expected performance}

Fig. [17 shows the gain in sensitivity expected for ALPS-II compared to ALPS-I. The sensitivity for the CCD improves approximately as $t_{\text {meas }}^{1 / 8}$ because it is dominated by the accumulated dark current. The TES is dominated by the statistics of the signal, hence the improvement is approximately $\propto t_{\text {meas }}^{1 / 4}$.

A summary of the expected detector performances and the resulting gain in sensitivity is given in Tab. 2 for a measurement campaign of two weeks corresponding to $336 \mathrm{~h}$ of data for the CCD and $168 \mathrm{~h}$ for the TES ${ }^{15}$.

In the following, details are given for both the CCD and the TES setup.

\footnotetext{
${ }^{14}$ The compressor could be a possible source of ground vibrations. Dampening these vibrations (in a first stage by a neoprene mat) and moving the ADR further away from the laser setup using a long optical fiber is possible, if these steps prove necessary for the locking of the laser cavity.

${ }^{15}$ This assumes that the cryogenic environment for the TES can be maintained $50 \%$ of the time.
} 

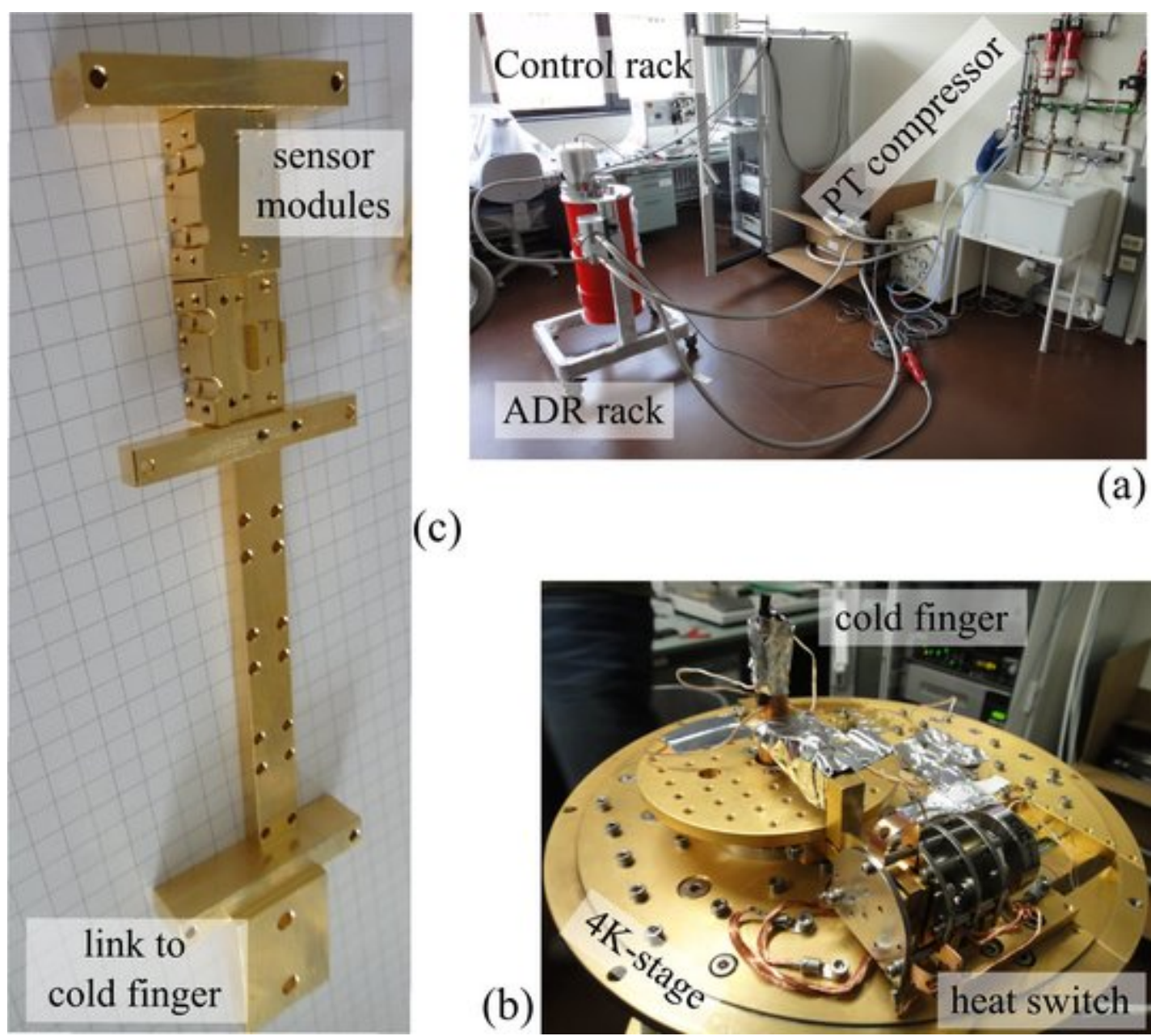

(c)

(a)

(b)

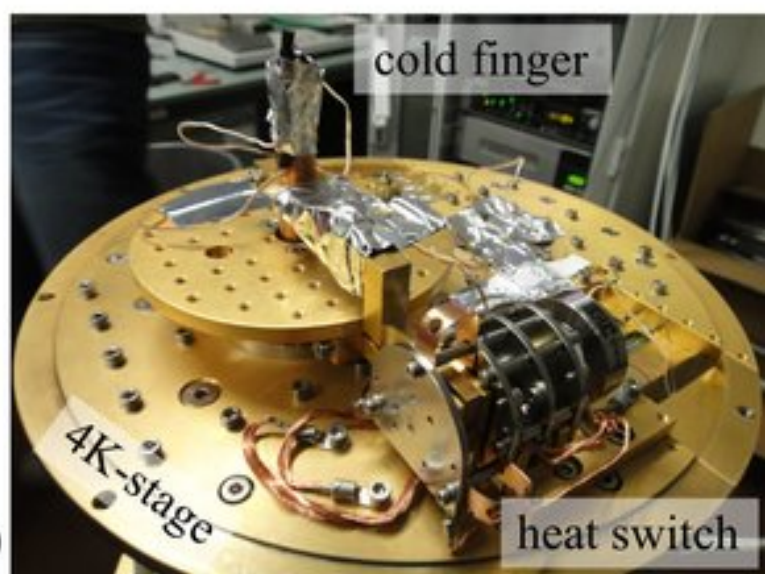

Figure 16. Status of ADR system and components: The pictures show the status at PTB, Berlin at the end of August 2012. (a) Infrastructure and components of ADR: The ADR itself (red dewar) is connected to the control rack for monitoring temperatures and pressure, operating the magnet and driving the heat switch. It is also connected to the Helium compressor of the pulse-tube cooler via $20 \mathrm{~m}$ pipes filled with Helium. (b) Open ADR: On the $4 \mathrm{~K}$ stage the heat switch is mounted, to connect and disconnect the $4 \mathrm{~K}$ bath to the salt pill. On top the cold finger is located reaching $\mathrm{mK}$. (c) Detector bench: The copper components are ready for attaching the sensors to $\mathrm{mK}$ bath. One sensor module is in Japan for attaching AIST chips

\begin{tabular}{l|c|c|c|c} 
Parameter & Impact & ALPS-I CCD & ALPS-II CCD & TES \\
\hline Efficiency $Q E$ & $g_{a \gamma} \propto Q E^{-1 / 4}$ & 0.9 & 0.012 & 0.75 \\
Detector noise $D C$ & $g_{a \gamma} \propto D C^{1 / 8}$ & $0.0018 \mathrm{~s}^{-1}$ & $0.0012 \mathrm{~s}^{-1}$ & $0.000001 \mathrm{~s}^{-1}$ \\
\hline \hline Sensitivity gain & & 1 & 0.5 & 2.4
\end{tabular}

Table 2. Parameters of the ALPS-II detector setups and their impact on the sensitivity of the experiment for a wave length of $1064 \mathrm{~nm}$ compared to the ALPS-I setup for $532 \mathrm{~nm}$ and $27 \mathrm{~h}$ of data. For the ALPS-II sensitivities two weeks of measurement are assumed. A sensitivity factor larger than 1 means an improvement of the sensitivity. Note that the detector noise for the TES remains to be demonstrated. 


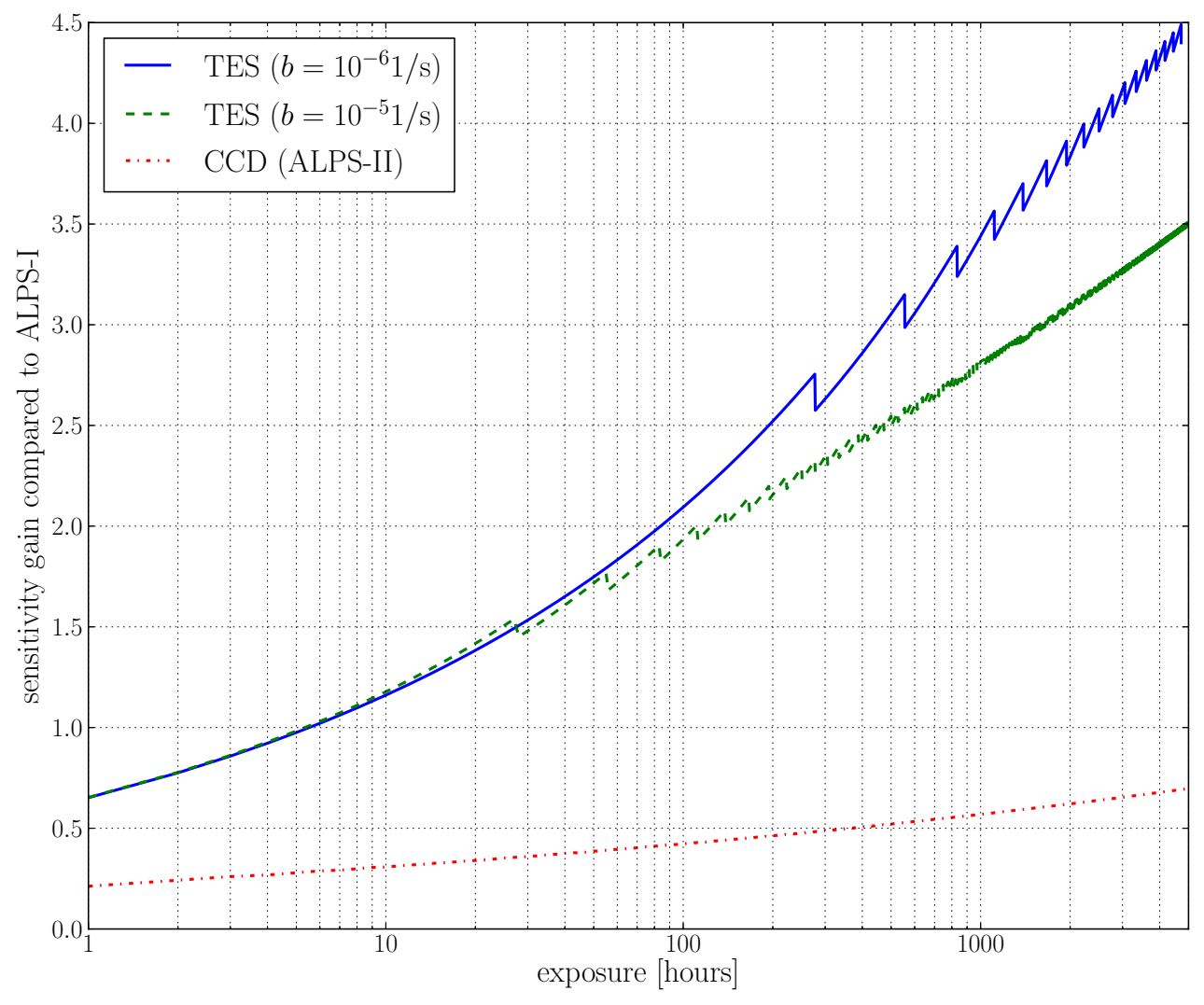

Figure 17. Expected sensitivity gain of the different detector systems for ALPS-II compared to ALPS-I: The dependency of the sensitivity gain on the exposure time is shown for the TES with a detection efficiency of $75 \%$ and background rates of $10^{-6} \mathrm{~s}^{-1}$ and $10^{-5} \mathrm{~s}^{-1}$, respectively, and for the CCD for the parameters given in Tab. ․ The number of observed background events was estimated conservatively by taking the smallest integer larger than the expected value for the respective measurement time (i.e., always $\geq 1$ ). The kinks in the TES lines are due to accumulation of additional background events. The comparison is made against the ALPS-I results for an exposure time of $27 \mathrm{~h}$ (cf. [四]).

\section{CCD:}

The dark current of the CCD is known from the data sheet and the experience in ALPS-I to be $0.0008 e^{-1}$ per pixel. The read-out noise has been determined ${ }^{16}$ to be $4.09 e$ at $100 \mathrm{kHz}$ readout speed and a quantum efficiency $1.2 \%$ was measured for a wavelength of $1066.7 \mathrm{~nm}$ and a chip temperature of $-70^{\circ} \mathrm{C}$. Fig. 18 shows the quantum efficiency as stated by the data sheet [100] together with the measured value.

Tests showed that the CCD can be operated reliably with exposure times up to $6000 \mathrm{~s}$. For longer observation times, the probability of contaminating the frame with charged particle background (cosmics and decay products) leads to a loss of frames.

In ALPS-I the end-mirrors of the production cavity had a limited lifetime and, thus, had to

\footnotetext{
${ }^{16}$ This value has been established in measurements and differs slightly from the value given by the manufacturer $(3.85 e)$.
} 


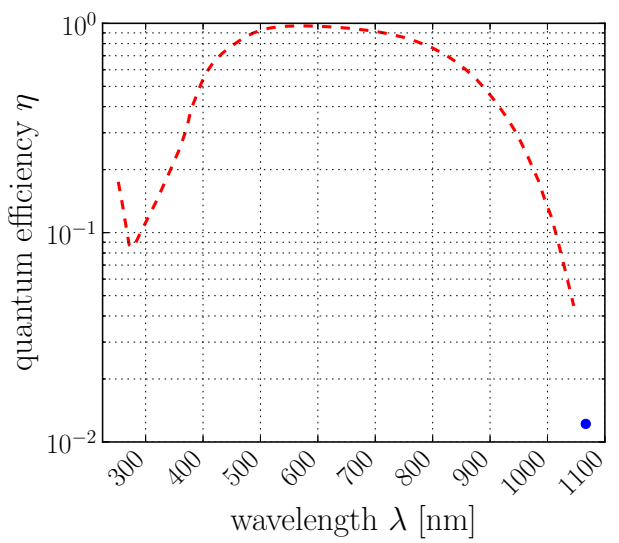

Figure 18. Measurement of the quantum efficiency of the PIXIS 1024B: The dashed line is the quantum efficiency measured at a temperature of $\mathrm{T}=25{ }^{\circ} \mathrm{C}$ taken from the data sheet [100]. The single bullet is the measurement of the quantum efficiency at $\mathrm{T}=-70^{\circ} \mathrm{C}$.

be replaced between measurements. Therefore, the position of the signal region changed between data runs and its shape had to be optimized with a time-consuming procedure resulting in a minimal achieved size of $3 \times 3$ pixel. During all phases of ALPS-II, the position of the signal region will be fixed. Hence, we expect to achieve a smaller signal region of $1 \times 1$ pixel.

\section{TES:}

For the setup using a Transition-Edge Sensor an intrinsic background level of less than $10^{-4}$ counts per second can be achieved. Only upper limits have been set by previous studies [90, 101] and a reduction up to rates as low as $10^{-7}$ counts per second might be possible as estimated for the ALPS setup based on $[101]^{17}$.

The quantum efficiency of the TES sensor was shown to be as high as $98 \%$ and of a whole detection setup of $95 \%$, if optimized [21]. Within the ALPS environment and especially the different fiber couplings described above, a detection efficiency of 60 to $80 \%$ is expected.

The energy resolution will be about $\frac{E}{\Delta E}=5$ to 6 , which could improve the background reduction in principle. A good timing resolution of about $1 \mu$ s easily enables selecting events based on, e.g., shutter conditions.

\footnotetext{
${ }^{17}$ For the calculation of the sensitivity gain compared to the detector setup in ALPS-I in Tab. 2, a background level of $10^{-6}$ counts per second is assumed.
} 

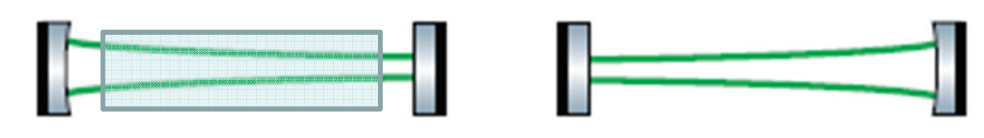

Figure 19. Shape of the photon beam in the optical cavity within the surrounding vacuum pipe. The vacuum pipe is only shown on one side of the setup (left).

\subsection{Magnets and cryogenics}

\subsubsection{Introduction}

To increase the sensitivity for the detection of axion-like particles, the ALPS-II collaboration plans to set up optical cavities both on the production and the regeneration side [84, 85] of the experiment with a power buildup of 5000 and 40000, respectively, and magnet strings of superconducting HERA dipoles as long as possible, as the sensitivity for the detection of axion-like particles scales with the product of magnetic field strength $B$ and magnetic length $L$ [20, 102, 56, 57], see also Eq. 2.1.

The achievable power buildup of an optical cavity with a certain length inside a string of dipoles depends on the aperture of the vacuum pipe in the dipoles [103]. The diameter of the circulating photon beam near the focusing mirror will be larger for larger lengths of the optical resonator (see Fig. 19). Therefore the clipping losses in the resonator will increase with length, limiting the number of dipoles per string for a given aperture.

Tab. 3 shows the maximum number of HERA dipoles, allowing for an optical cavity with a power buildup of 40000 at a wavelength of $1064 \mathrm{~nm}$ for different apertures. The free aperture is taken to be $10 \mathrm{~mm}$ smaller, to account for alignment tolerances, the waviness of the vacuum pipe, and fluctuations of the laser position. For comparison the apertures and the values for $B \cdot L$ for an experimental setup using LHC dipoles [104] as proposed in [85] or Tevatron dipoles [105], as considered for REAPR [106-108] are included.

The inner diameter of the vacuum pipe in the superconducting HERA dipole is $55 \mathrm{~mm}$. Due to the curvature of the dipoles built for HERA ${ }^{18}$, the free horizontal aperture is reduced to $\approx 35 \mathrm{~mm}$. However, as will be described below, HERA dipoles can be straightened by a modification of the cold mass. This modification yields the full aperture of $55 \mathrm{~mm}$ and allows for the best possible setup for ALPS-II of $2 \cdot 12$ dipoles. However, the total costs of this operation, although not known at present, are expected to be considerable.

Therefore a cheaper way to increase the aperture in the dipoles has been investigated (see below). By a "brute force" deformation of the magnet yoke an aperture of 50mm (almost straight) can be obtained, allowing for a setup of $2 \cdot 10$ dipole with a power buildup of 40000 . This is the base line layout for the ALPS-IIc proposal.

\footnotetext{
${ }^{18}$ See for example [109]. Key people for the development of the superconducting HERA magnets were H. Kaiser, K.H. Mess, P. Schmüser and S. Wolff.
} 


\begin{tabular}{|c|c||c|c|c|}
\hline \multicolumn{2}{|c|}{$\begin{array}{c}\text { Dipole aperture } \\
\text { mm }\end{array}$} & Number of dipoles & $B \cdot L[\mathrm{Tm}]$ & $\begin{array}{c}\text { Length of single string } \\
\text { plus 5m optical setup }\end{array}$ \\
\hline \hline 35 & $\mathrm{HERA}^{19}$ & $2 \cdot 4$ & 187 & 44 \\
\hline 40 & $\mathrm{LHC}^{20}$ [85] & $2 \cdot 4$ & 515 & 72 \\
\hline 48 & REAPR $^{21}[108]$ & $2 \cdot 6$ & 184 & 44 \\
\hline 50 & $\begin{array}{c}\text { HERA } \\
\text { almost straight }\end{array}$ & $2 \cdot 10$ & 468 & 103 \\
\hline 55 & $\begin{array}{c}\text { HERA } \\
\text { straight }\end{array}$ & $2 \cdot 12$ & 562 & 122 \\
\hline
\end{tabular}

Table 3. Comparison of $B \cdot L$ for HERA dipoles with different apertures and proposals using LHC or Tevatron dipoles.

\begin{tabular}{|c|c|c|c|c|}
\hline Experiment & $\begin{array}{c}\text { Year of } \\
\text { publication }\end{array}$ & $\begin{array}{c}\text { Magnetic length } \\
L[\mathrm{~m}]\end{array}$ & $\begin{array}{c}\text { Magnetic field } \\
B[\mathrm{~T}]\end{array}$ & $B \cdot L[\mathrm{Tm}]$ \\
\hline \hline BFRT [17] & 1993 & 4.4 & 3.7 & 16.3 \\
\hline BMV [15] & 2007 & 0.25 & 11 & 2.8 \\
\hline LIPPS [10] & 2008 & 1 & 1.7 & 1.7 \\
\hline PVLAS [110] & 2008 & 1 & 5 & 5 \\
\hline GammeV [11] & 2008 & 3 & 5 & 15.0 \\
\hline ALPS-I [6] & 2010 & 4.42 & 5.0 & 22.1 \\
\hline OSQAR [14] & 2011 & 14.3 & 9 & 128.7 \\
\hline
\end{tabular}

Table 4. Values of $B \cdot L$ for previous laboratory experiments.

It should be noted that already a setup for ALPS-IIc with $2 \cdot 4$ standard HERA dipoles would result in a larger sensitivity with respect to $B \cdot L$, compared to laboratory experiments performed up to now ${ }^{22}$ (see Tabs. 3 and $\bigoplus$ ). With straight HERA dipoles and also with the almost straight dipoles ALPS-IIc will be clearly competitive to the other experimental setups proposed with respect to $B \cdot L$.

The infrastructure needed for the operation of a superconducting dipole string is specific for each type of dipole, i.e., the LHC, HERA, or Tevatron dipole. This refers for example to the cryogenics, the cryogenic boxes, the power supply, or the quench protection system. Although it is possible to set up a string of the three types of superconducting dipoles, considered here at any major laboratory, the effort needed would be substantially larger compared to the "home" laboratory of the magnet. Therefore we did not consider the use of LHC or Tevatron dipoles on the DESY site.

\footnotetext{
${ }^{19}$ HERA: magnetic length $8.83 \mathrm{~m}$, field $5.3 \mathrm{~T}$.

${ }^{20}$ LHC magnetic length $14.3 \mathrm{~m}$, field $9 \mathrm{~T}$.

${ }^{21}$ REAPR magnetic length $6.12 \mathrm{~m}$, field $5 \mathrm{~T}$.

${ }^{22}$ As detailed in Sect. 6 2 $2 \cdot 10$ magnets at the envisaged power build-up suffice to improve current helioscope limits and tackle parameter regions favored by theory and astrophysical hints.
} 


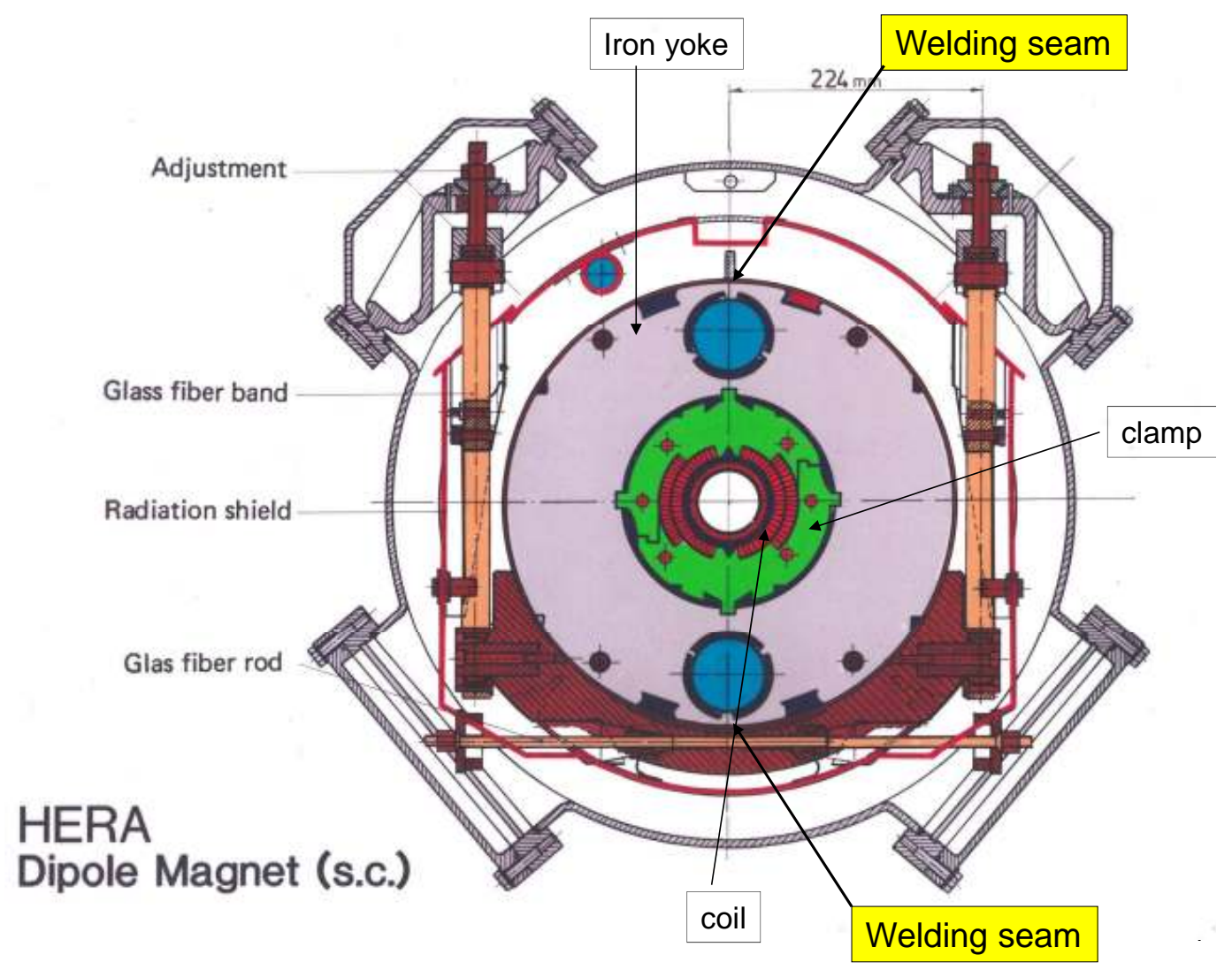

Figure 20. Cross section of the HERA dipole cryostat. The welding of the half cylinders of the Helium vessel around the iron yoke was performed in a big tool which forced the cold mass to a given curvature. The beam pipe was forced to follow the curvature by spacers glued to the outside of the pipe.

\subsubsection{Straight HERA dipoles}

Originally the iron yoke, the clamps, the coils, and the vacuum pipe of the HERA dipole were fabricated straight; only the outer vacuum vessel was formed as a polygon. The welding of the half cylinders of the Helium vessel around the iron yoke was performed in a big tool which forced the cold mass to a given curvature. The beam pipe was forced to follow the curvature by spacers glued to the outside of the pipe.

Therefore by cutting the welding seam of the Helium vessel (see Fig. 20), straightening the yoke and welding two straight half cylinders around the yoke, it should be possible to obtain a straight dipole magnet. However, this procedure requires the complete disassembly of the magnet cryostat and the rebuilding of part of the tooling used originally. The know-how for this procedure still exists in one company which originally assembled half of the total number of dipoles. The cost for this method of straightening HERA dipoles however, although not known at present, is expected to be considerable.

We therefore looked for a simpler and more importantly cheaper way of straightening the dipole. Engineering studies showed that a straightening of the yoke and thus the beam pipe should be possible by a brute force deformation with $\approx 40 \mathrm{kN}$ from the outer vacuum vessel at the 3 planes 

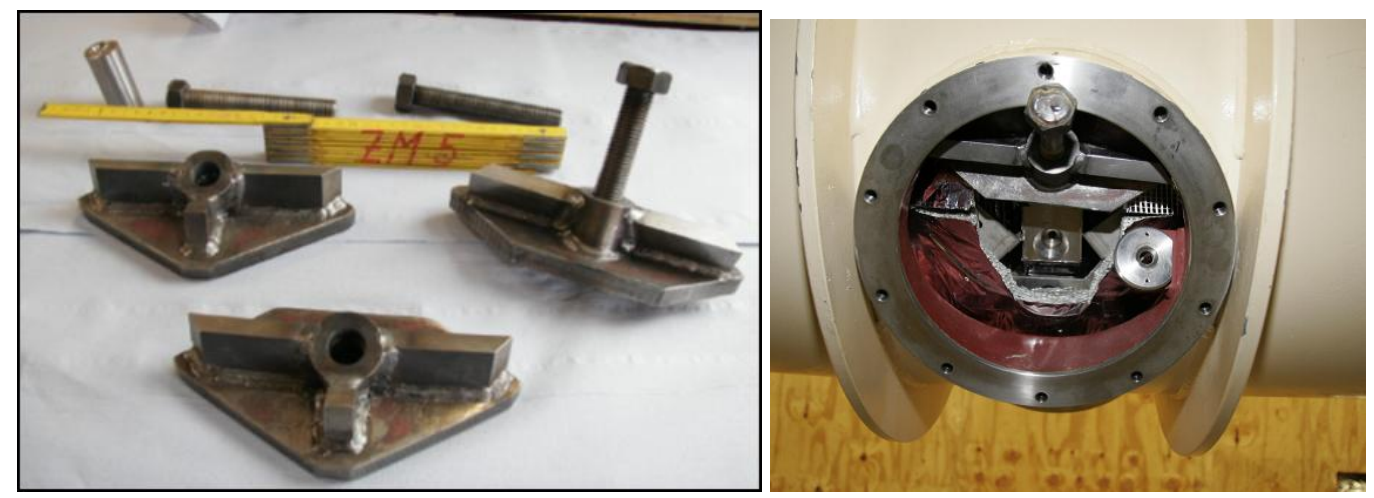

Figure 21. Tools for straightening the cold mass (left) and the inserted tools (right).

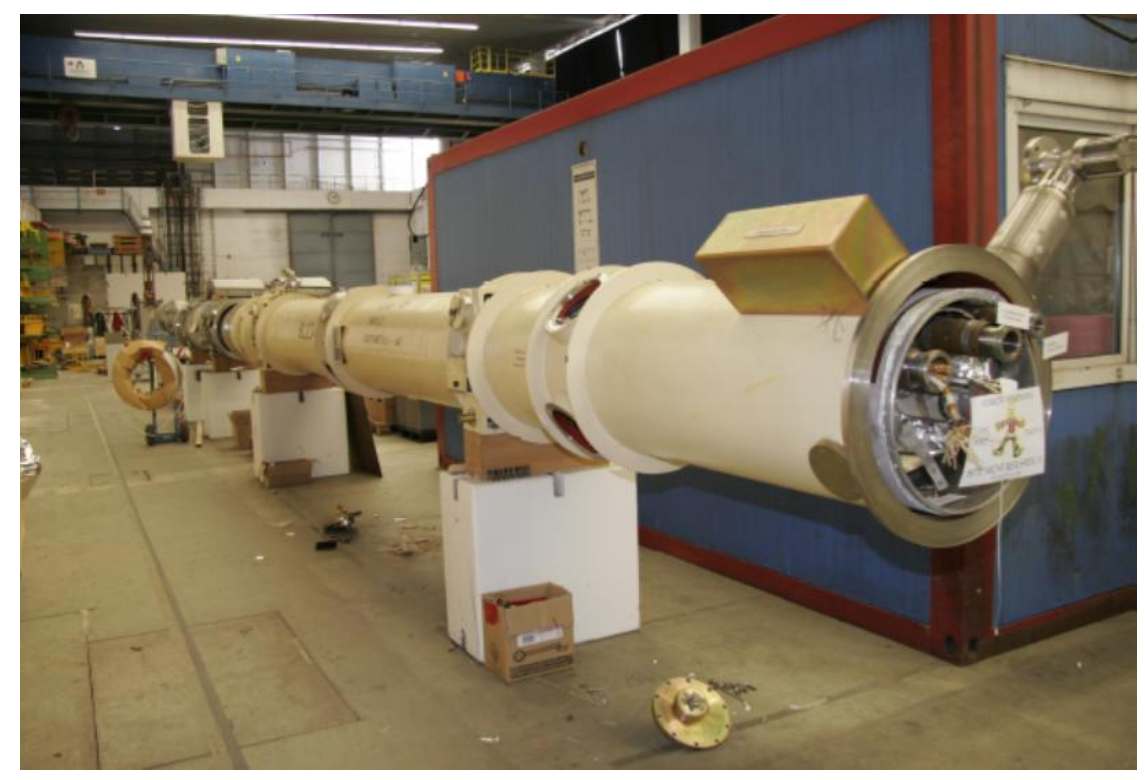

Figure 22. Setup of a HERA dipole to test the deformation method. The dipole was used as an exhibit for many years and was not foreseen as a spare for HERA.

of support of the dipole ${ }^{23}$.

In May 2011 the deformation method was first tried on a HERA dipole serving as an exhibit before. The tools to bend the magnet yoke (see Fig. 21) were inserted into the lower flanges of the outer vacuum vessel at three positions, near the ends of the dipole on one side of the vessel and in the middle on the other side (see Figs. 21 and 22).

Fig. 23 shows the position of the center of the beam pipe before and after the application of the deforming forces. The deviation from a straight line was reduced ${ }^{24}$ from $16.24 \mathrm{~mm}$ to $5.24 \mathrm{~mm}$, yielding about $90 \%$ of the maximum horizontal aperture of $55 \mathrm{~mm}$.

This result proves the applicability of the "brute force" method to substantially enlarge the

\footnotetext{
${ }^{23}$ Concept by Rüdiger Bandelmann; development by Gerhard Meyer. We learned later from [85] that a similar method had been considered at CERN by P. Pugnat for LHC dipoles.

${ }^{24}$ Measurements of the beam pipe were performed by the DESY survey group MEA.
} 


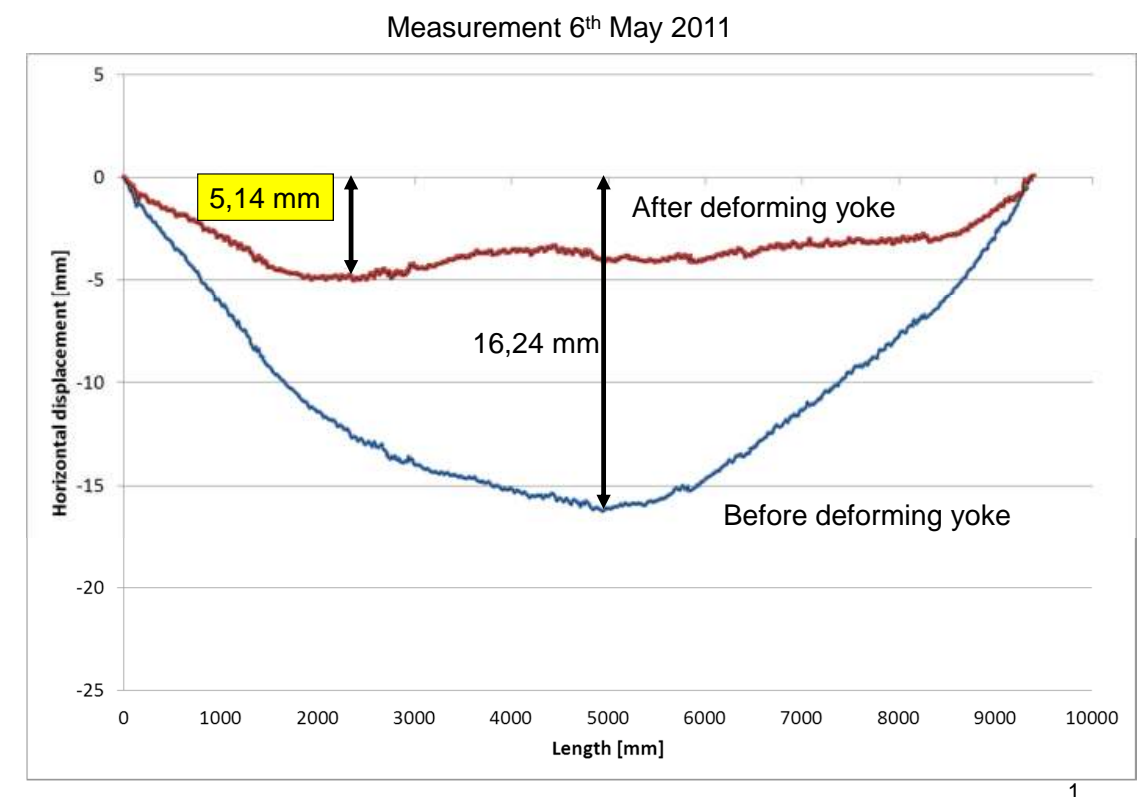

Figure 23. Position of the beam pipe axis along the magnet.

horizontal aperture of the vacuum pipe of the superconducting HERA dipoles.

As the deformation of the yoke is elastic, the deforming force has to be maintained during operation at cryogenic temperatures. Therefore pressure props were designed, which keep the thermal flux from the vacuum vessel at room temperature to the yoke at liquid Helium temperature within acceptable limits. The pressure props replace the deformation tools after the straightening (see Fig. 24).

The props near the ends of the dipole must allow for the length change of the yoke during cooldown and warm-up and yet maintain the deforming pressure. A simple solution to this problem is to use a section of a sphere which rolls with the motion of the cold mass ${ }^{25}$. This way the distance between the vacuum vessel at room temperature and the yoke does not change during the thermal motion except for the small thermal shrinkage of the sphere. The forces are always perpendicular to the surfaces thus avoiding a momentum on the prop and the danger of its tipping over. The thermal heat flow from room temperature to the cold mass at $4 \mathrm{~K}$ through the thin walled titanium tube of the pressure prop, see Fig. 25, amounts to about 1 Watt per prop.

A prototype pressure prop was subjected to functional tests in vacuum and at liquid Nitrogen temperature to make sure about the proper choice of materials and the validity of the concept. The test validated the concept and the choice of materials.

For the insertion of the deforming tools, 3 of the total 6 suspensions of the cold mass and the radiation shield have to be removed. The installed pressure props interfere with a re-installation of the suspensions. While the cold mass is still well supported by the three remaining suspensions,

${ }^{25}$ Concept and realization by Gerhard Meyer. 


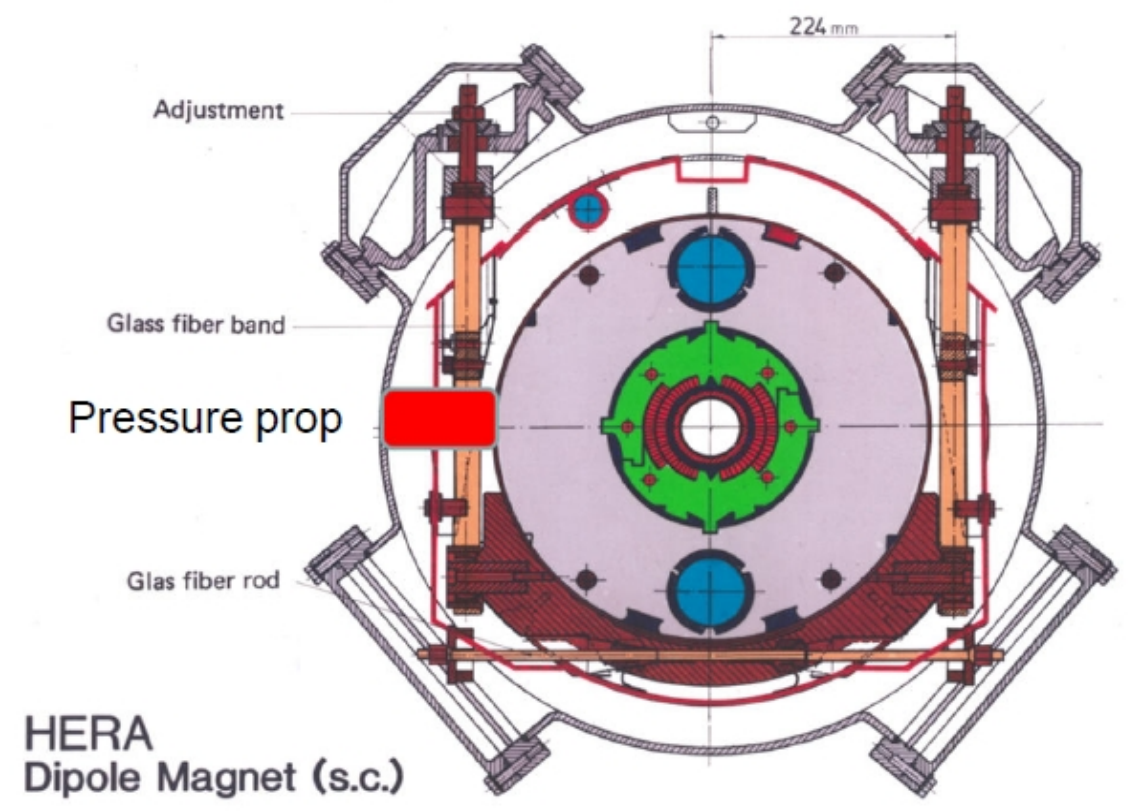

Figure 24. Position of a pressure prop in the dipole cryostat.

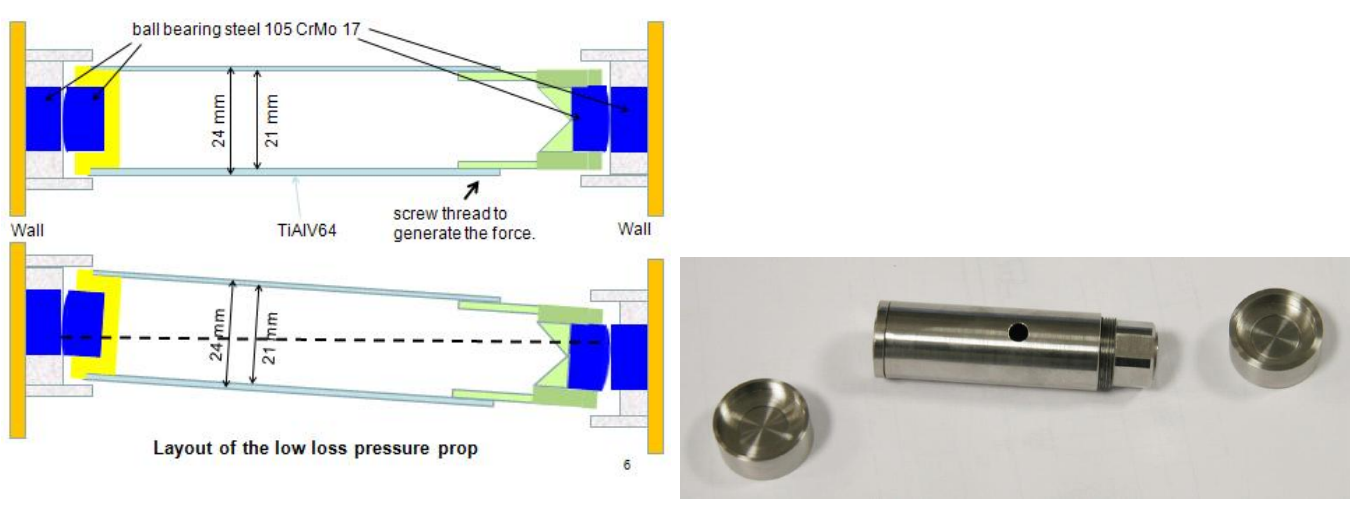

Figure 25. Principal layout of a pressure prop with low thermal conductivity and the first prototype.

the radiation shield, being a rather soft structure, needs to be supported. By replacing the G10 (glas fiber enforced epoxy) loops of the suspensions by steel strips (see Fig. 27), connected to the shield part of the suspension off center, and by machining a slit into the shield, to give room for the pressure props during installation, the suspension of the radiation shield can be reestablished in essentially the same way as before.

Recently (August 2012) the HERA dipole at the magnet test bench in hall 55, which was used for the ALPS-I experiment (see Fig. 26), has been straightened on the test bench, with the Helium 


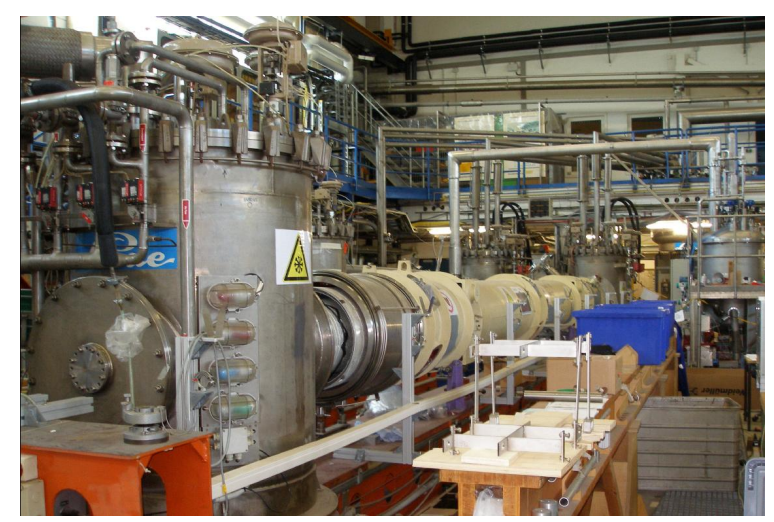

Figure 26. Test bench with the HERA dipole used for the ALPS-I experiment. It is located in hall 55 and will be used to test all dipoles foreseen for ALPS-IIc.
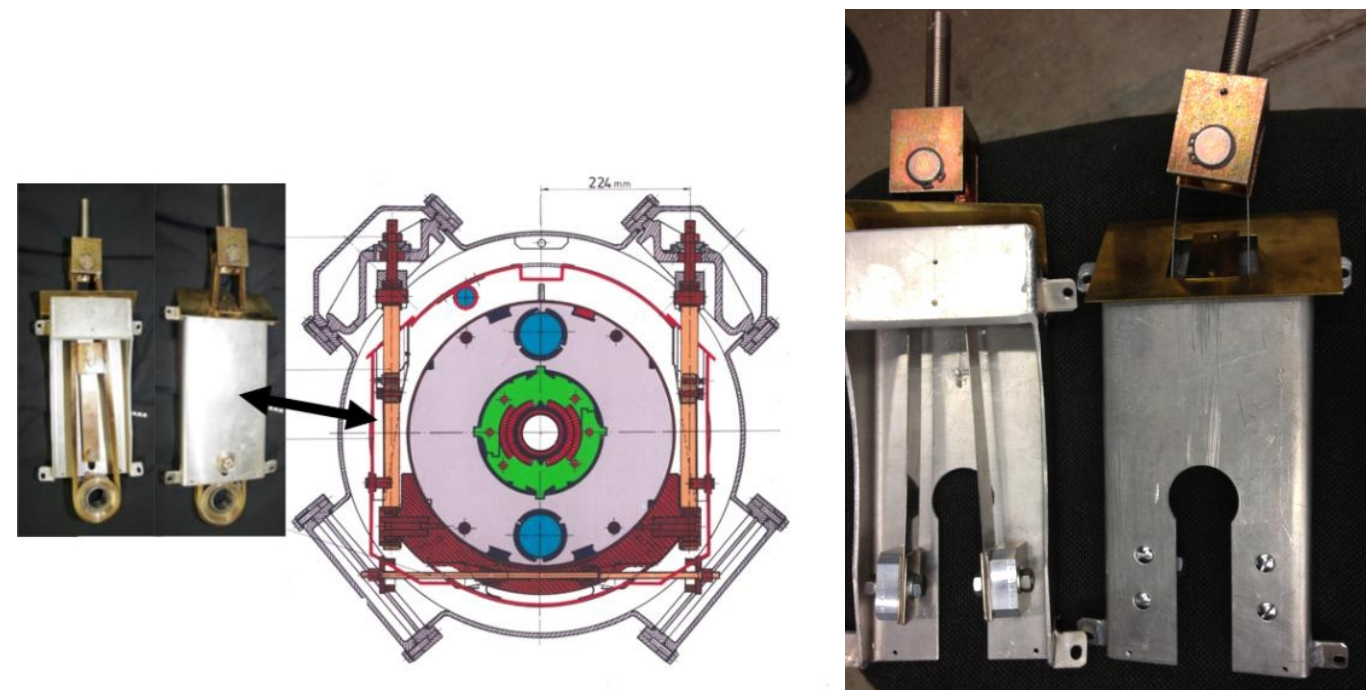

Figure 27. Suspension of radiation shield and the modification to allow the insertion of a pressure prop.

pipes of the cryogenic boxes remaining connected to the dipole.

The straightened dipole was operated in September 2012 at cryogenic temperatures reaching a quench current of 6050 amperes $^{26}$. This current is slightly higher than the quench current measured on the unmodified dipole (5920 amperes) in August 2011. The straightened dipole was operated for 30 hours continuously at the design current for ALPS-IIc of 5700 amperes. The cryogenic losses of the straightened dipole were as for the unmodified dipole.

The straightening procedure will be reviewed based on the experience gained during the first straightening experiments. Better assembly tools will have to be developed, as the correct positioning of the pressure props turned out to be difficult. After the testing of the new tools on a magnet, pressure props will be fabricated to start the straightening of the magnets foreseen for the ALPS-IIc strings. Measurements of the position of the vacuum chambers along the dipoles (see below) will allow for a selection of their location in the magnet strings, which yields the largest overall aperture

\footnotetext{
${ }^{26}$ The measurements were performed by members of the DESY group MKS.
} 


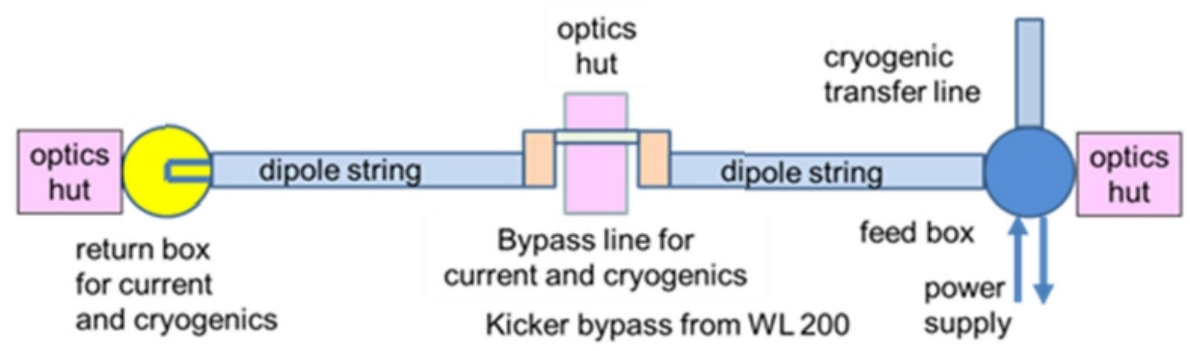

Figure 28. Schematic layout of the experimental setup for ALPS-IIc. Cold Helium and the magnet current are fed into a cryogenic box on one side of the experiment and run through both dipole strings to a cryogenic box at the other end of the experiment setup, where the flow is directed back through the dipole strings. Helium and electrical current are led around the optical setup in the middle by a bypass line. Please note that all optical elements are placed outside the cold part of the experiment and thus are not expected to be affected by the cool-down of the magnets.

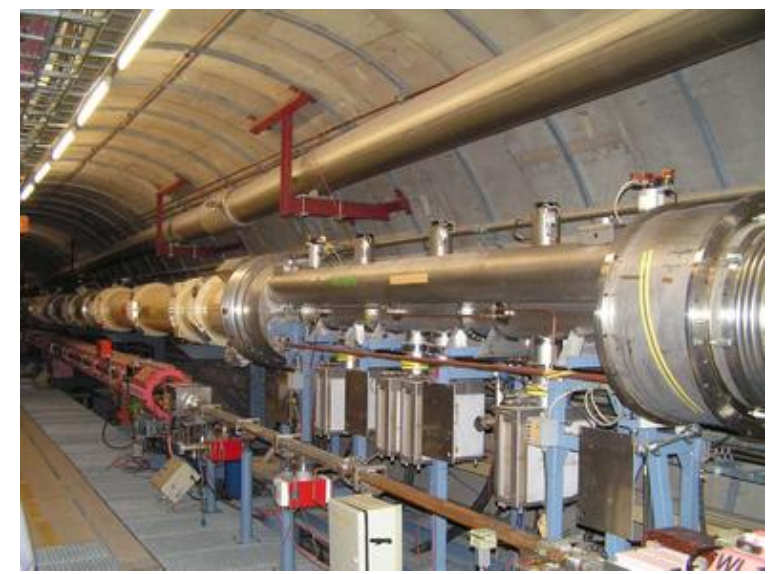

Figure 29. Kicker bypass in the HERA tunnel (West left $200 \mathrm{~m}$ ). The bypass will be installed in the middle of the experiment connecting the two dipole strings of ALPS-IIc and pass the magnet current and the cryogenics around the optical setup.

for the optical resonators.

\subsubsection{The ALPS-IIc magnet string}

A string of $2 \cdot 10$ HERA dipoles, straightened with the described deformation method, will supply the necessary horizontal aperture for the optical cavities with sufficiently low clipping losses. Twenty-four spare HERA dipoles are available.

Fig. 28 shows the setup of the experiment with the two magnet strings and the optics huts schematically. The cryogenic boxes at the ends of the magnet strings are taken from the straight section of HERA selected for the setup of the experiment (see Sect. 3.8 for site considerations). To pass the magnet current and cryogenics around the optical setup in the middle, the "Kicker Bypass" 

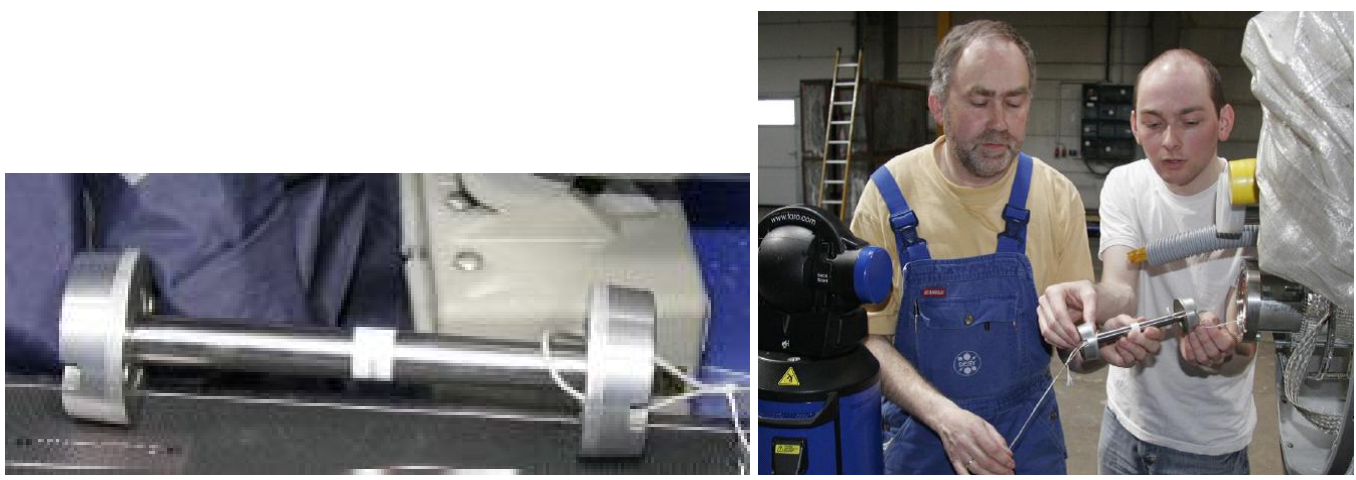

Figure 30. The "mouse" survey tool, used to measure the position of the middle of the beam pipe along the magnet.

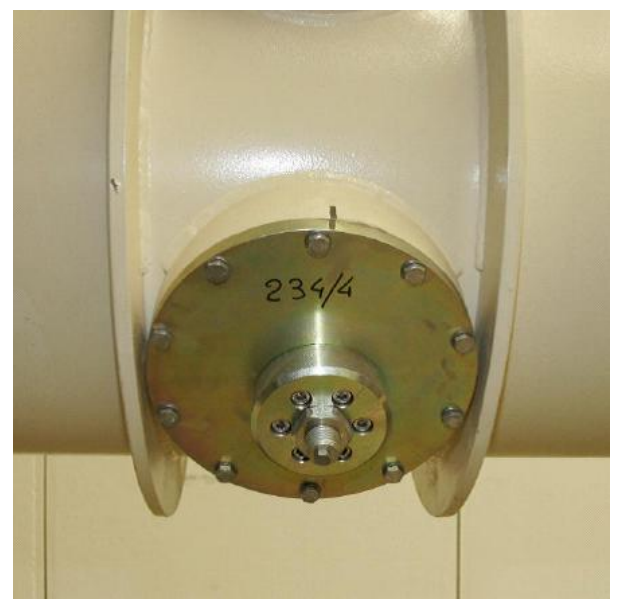

Figure 31. Transportation fixture.

from the HERA section WL 200 will be used (see Fig. 29). Essential systems like the quench gas collection line, other warm Helium pipes or the dump resistors are not shown.

\subsubsection{Alignment}

The position of the beam pipe along the magnet will be measured for each dipole. This will be done using a simple tool called "mouse" 27 , which is pulled through the pipe with a string (see Fig. 30). From the data, the positioning of each dipole can be evaluated yielding the largest horizontal aperture in the string of magnets. This information will be transferred to existing survey marks on the outer vacuum vessel to allow for the proper positioning of the magnet in the HERA tunnel.

The dipoles foreseen for ALPS-IIc are stored outside the HERA tunnel in a hall on the DESY site. For the transportation of the dipoles, the magnet yoke has to be fixed to the outer vacuum vessel by special fixtures (see Fig. 31) to avoid damage to parts connecting the cold mass with the vacuum vessel, like the magnet and shield suspensions, or pipes containing the cables for the quench detection. This is especially important for the transport into the HERA tunnel, as the magnet has to be rotated out of the horizontal to fit through the access shaft. It remains to be

\footnotetext{
${ }^{27}$ Measurements of the beam pipe were performed by the DESY survey group MEA.
} 
measured, whether the insertion of the fixtures and the transportation of the magnet change the position of the beam pipe.

The design of the supports of the Helium vessel guarantees that the position of the middle of the magnet with respect to the outer vacuum is the same at room temperature and at the operating temperature of $4 \mathrm{~K}$. However, it remains to be measured whether this also holds for the position of the beam pipe along the magnet. This measurement can be performed at the magnet test bench, where the achievable magnetic field will be measured for every dipole before the installation into the HERA tunnel. It is conceivable, to use the "mouse" on the test stand by pulling the string by stepping motors in the insulating vacuum. However, the method suited best for this measurement remains to be determined.

It should be noted that the dipoles can be moved within the completed setup horizontally and vertically by a few millimeters, even when the string is operated at liquid Helium temperature.

\section{Power Supply for the Magnet String of ALPS-IIc}

To operate the dipole strings at a magnetic field of $5.3 \mathrm{~T}$ a power supply has to deliver a current of $5690 \mathrm{~A}$ at a voltage given by the resistance of the cable connections between the power supply in the hall and the magnet string in the tunnel. For the existing connection of the HERA superconducting magnet ring in hall West with a resistance of $4 \mathrm{~m} \Omega$ the voltage amounts to $\approx 23$ Volt at full current.

The power supply has also to supply additional voltage during the ramp up of the current due to the inductive voltage $L \cdot \mathrm{d} I / \mathrm{d} t$ at the magnet string. A ramp rate of $2.6 \mathrm{~A} / \mathrm{s}$, corresponding to a ramp time of 36.5 minutes, would require an additional voltage of $\approx 3$ Volts at the power supply for a string of $2 \cdot 10$ dipoles.

An adequate power supply is available in hall West, the original power supply for the superconducting magnets of the HERA proton ring. In principle this power supply can be moved to any of the three other halls. However, operation of the power supply at the required values, at HERA halls other than hall West, would need investments for additional transformers and cabling [11]].

This expenditure can be reduced using the power supply in hall North, originally used for the operation of the superconducting solenoid of the H1-experiment. By reducing the cable resistance between the power supply and the magnet strings to below $3 \mathrm{~m} \Omega$ the capacity of the power supply of $6000 \mathrm{~A}$ at 20 Volts is sufficient to ramp the current and operate a string of $2 \cdot 10$ dipoles. There is an excellent proposal [11]] to achieve this reduced resistance, by using the main bus bar system of the HERA electron dipoles in parallel to the cables from the proton ring.

\section{Quench Protection}

If a quench happens in one of the dipoles, the current in the coil must be reduced to zero in a short time $(<1 \mathrm{sec})$ to avoid overheating and possibly a destruction of the normal conducting part of the coil [112]. The solution used at HERA is to bypass each magnet in the chain with a diode; if a magnet quenches, the current in the chain is reduced slowly but is guided around the quenched coil (see Fig. 32) by means of the diode [112].

If a quench is detected, the power supply is switched off, and switches to dump resistors (stainless steel tubes of $57 \mathrm{~mm}$ diameter and $2.9 \mathrm{~mm}$ thick walls) are opened to dissipate the stored energy [113]. The current decays with the time constant $L / R$, where $L$ is the inductance of the magnet string (1.16 Henry) and $R$ is the resistance of the dump resistors plus the cable connections 


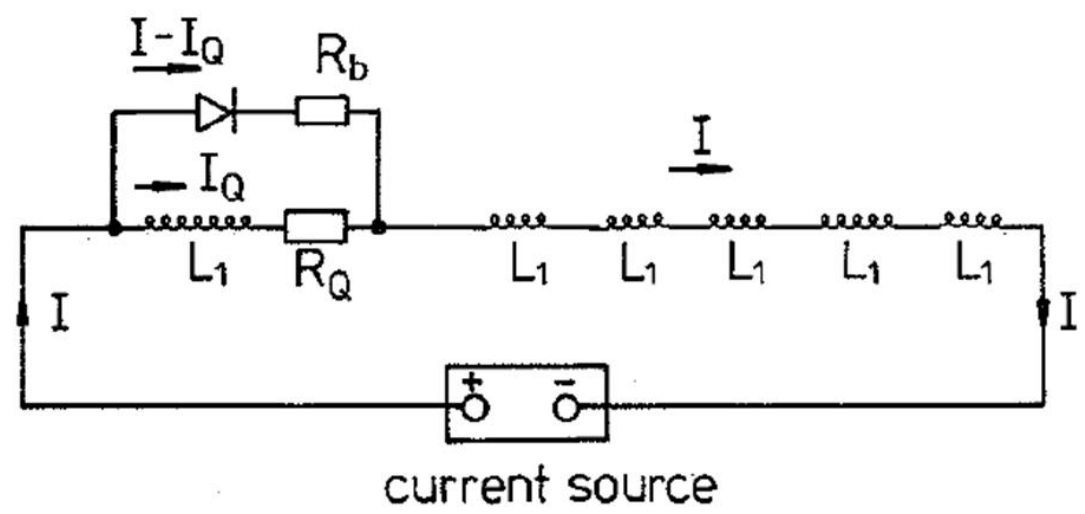

Figure 32. Schematics of the HERA quench protection.

to the power supply. Two dump switches increase the safety in case one switch fails and generate a symmetric voltage profile across the string. Requiring that the inductive voltage per individual dipole during the decay of the current be equal to the operation of HERA $(\approx 13$ Volt $)$, yields a value of $24 \mathrm{~m} \Omega$ for the resistance of the dump resistors, which can be realized by slight modifications to the existing dump resistors.

For the operation of the dipole strings of ALPS-IIc a new quench detection- and protectionelectronics system is required, as the components used for the HERA system are not fully functional anymore and spares for the outdated electronics and controls are not available. The work on this system was started in August 2011. It is planned to install and test a prototype of the new system at the magnet test bench setup in hall 55 at DESY in 2014. The complete quench protection system has to be available after the magnet string installation at the end of 2016. This leaves sufficient time for testing and fabrication of the complete system $(\approx 1$ year $)$.

\section{Magnet Strings with alternating polarity}

The current in the HERA dipoles can flow in only one direction due to the diodes. A string of dipoles with the standard interface connection between magnets can therefore supply only a single direction of the magnetic field. A segmentation of the magnetic field into regions of alternating polarity to increase the mass range of the experiment, as proposed in reference [114], is not possible without major efforts like special connection boxes between individual dipoles. However, a comparable increase in sensitivity can be achieved without much effort by changing the refractive index in the optical resonators (see Sect. 3.6, vacuum system).

\section{Cryogenics}

Supplying cold Helium to the ALPS-IIc setup is possible in principle to any of the HERA halls from the cryogenics plant on the DESY site [115, [16]. The effort and the cost of operation depend on the straight section chosen for the setup.

The purchase of a new smaller stand-alone refrigerator was considered, but has been discarded due to the high cost compared to the use of the DESY plant.

There are many advantages in supplying the ALPS-IIc magnet strings with cold Helium from the DESY cryogenics plant like a trained and experienced operations crew, or the availability of 


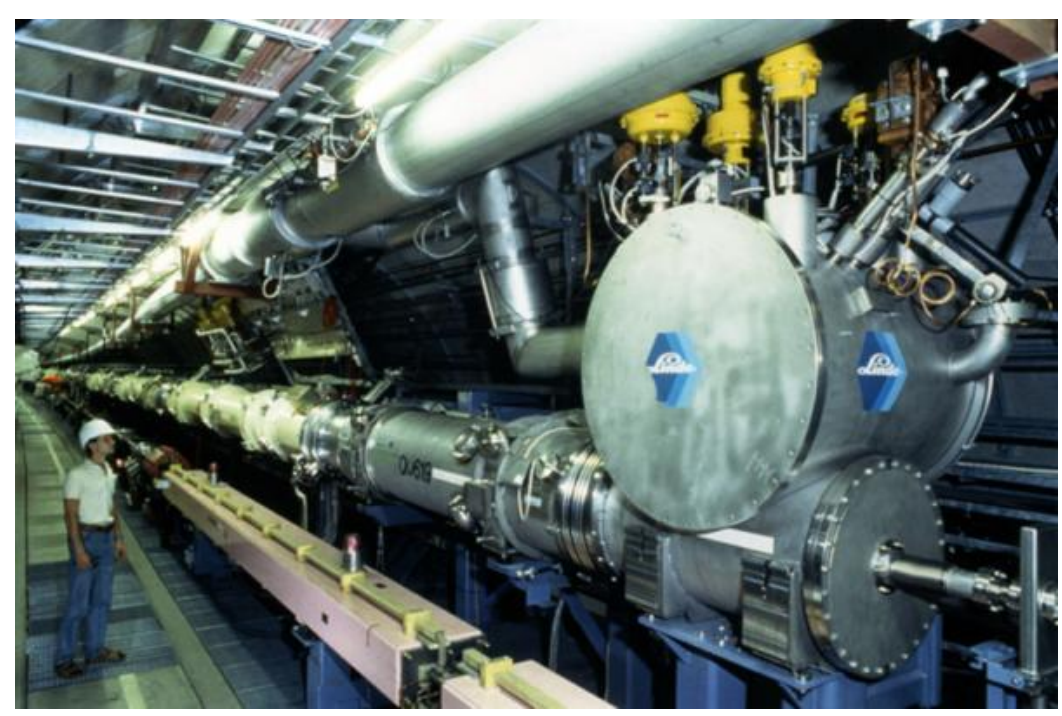

Figure 33. Cryogenic feed box for an octant of superconducting magnets at HERA.

large storage tanks for the Helium gas, when the strings have to be warmed up to room temperature (18 tanks with $367 \mathrm{~m}^{3}$ at 20 bar are available). The storage of $\approx 170 \mathrm{~m}^{3}$ required for the warm up of a $2 \cdot 10$ dipole string is therefore easily possible and -it should be noted- without any limitation to the warm up of the XFEL or FLASH accelerator modules with superconducting cavities.

For ALPS-IIc cold Helium will be transported from the cryogenics plant into the tunnel to a box (see Fig. 28) by the available Helium transfer line [117], which feeds the Helium into the strings at one side and returns the Helium to the plant after its passage through the strings. At the other end of the strings a similar box will be connected to the magnets, which returns the Helium and the current through the strings. It is intended to use the cryogenic boxes presently installed in the HERA tunnel at the end of the straight section chosen for the setup of ALPS-IIc. However, the boxes, as installed for HERA (see Fig. 33), have to be moved to the other side of the straight section to match the connection pattern of the dipoles. They must be disconnected from the Helium transfer line and other cryogenics equipment like pre-cooler or valve stations and be reconnected at their new position.

In case of a quench in the dipoles or a failure of the insulation vacuum, the Helium pressure in the magnets will increase and eventually has to be relieved before it reaches a level $(\approx 20$ bar $)$ which might damage the magnets. The Helium is released via an automatic valve (see right side of Fig. (33), named after its inventor Kautzky from Fermilab, into the quench gas collection tube (see Figs. 34 and 35), which guides the gas back to the cryogenics plant. In the valve a reference pressure and the pressure in the magnets are compared, thus defining a threshold for the pressure relief. The reference pressure is supplied from a high pressure line (see Figs. 34 and 35) and will be set to 14 bar like at HERA. The existing quench gas collection tubes and the high pressure lines can be used for ALPS-IIc by adding new connections to the Kautzky valves of the magnets in the strings.

To operate the magnet strings, pressure code regulations have to be met. The necessary pressure tests will be performed as for HERA. 


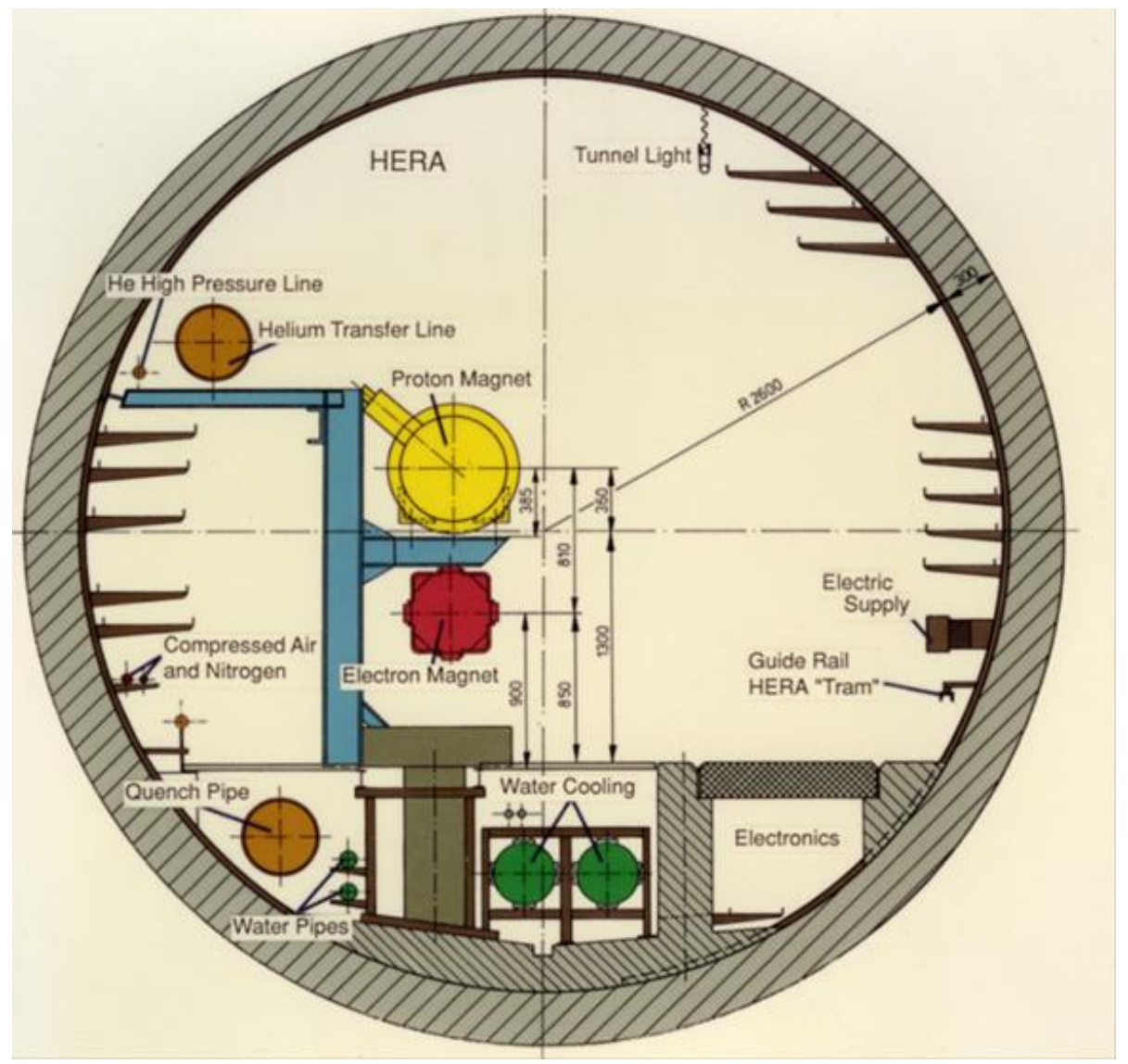

Figure 34. Cross section of the HERA tunnel in the arcs. The ALPS-IIc setup in the straight sections of the HERA tunnel will look very similar, the main difference being that the components of the HERA electron ring will have been dismantled.
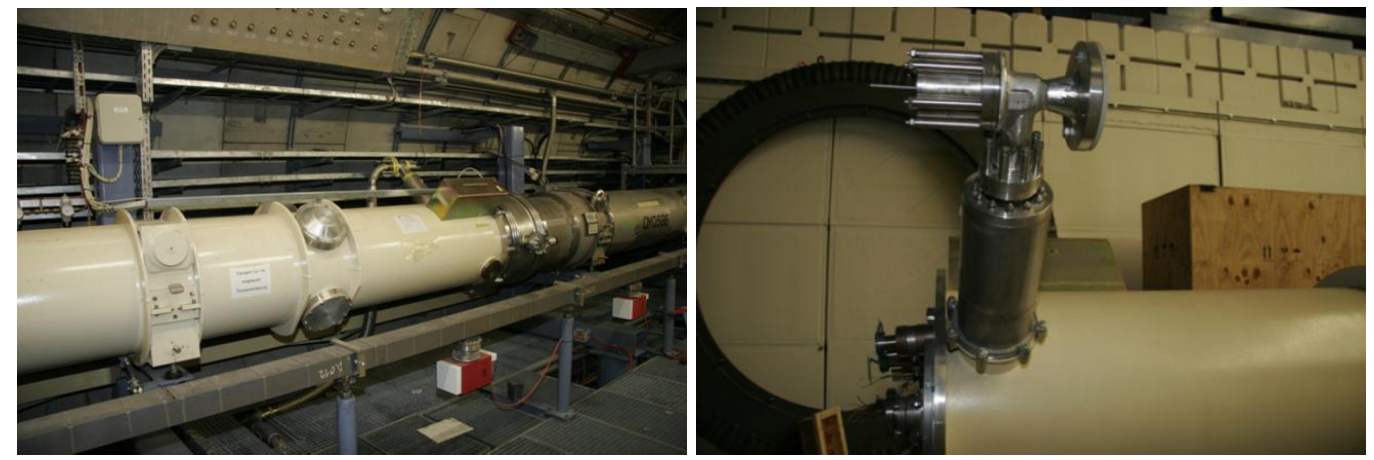

Figure 35. "Kautzky"-relief valve connected to the quench gas collection line (left) and "Kautzky"-relief valve (right).

The total heat load at the $4 \mathrm{~K}$ level of the straightened magnet strings for ALPS-IIc amounts to about 140 Watt, substantially less than the heat load of about 400 Watt for an octant, which is the smallest cryogenic unit of HERA's superconducting magnet ring.

As the clipping losses from the stored laser beam power of $150 \mathrm{kWatt}$ on the vacuum pipe 
aperture are well below 1 Watt in the entire magnet string, it can be concluded that the cryogenic operation of the dipole strings will not pose any problems.

\subsection{Vacuum system}

The vacuum system for the optical resonators of ALPS-IIb and ALPS-IIc and the insulating vacuum system for the superconducting magnet strings will be described. As we will outline below, here, we profit very much from the experience gained at HERA. In addition the concepts for changing the refractive index in the cold pipe within the optical resonators of ALPS-IIc will be described.

\subsubsection{The vacuum system for the optical resonators of ALPS-IIb}

In the straight section HERA West two optical cavities of about 100m length can be set up with very little effort needed for the vacuum system. The still existing vacuum pipe of the HERA proton ring can be used for ALPS-IIb as it is straight over about $2 \cdot 160 \mathrm{~m}$ in contrast to the other straight sections of HERA, where vertical bending magnets limit the available straight length in the vacuum pipe to about $2.60 \mathrm{~m}$. The aperture of the proton beam pipe is large (cf. Fig. 44) - except for one location which can be easily modified - and allows the operation of optical cavities at very low clipping losses.

Locations in hall West and in the tunnel on both sides of the hall have been identified, where little effort is required to remove the existing accelerator installation to free space for the cleanrooms at an adequate distance from the hall. The vacuum vessels for the optical components will be identical to the ones used at ALPS-IIc (see below).

The existing vacuum system is equipped with a large number of ion getter pumps and titanium sublimation pumps which will allow to obtain very low vacuum pressures in the optical resonators. At HERA a pressure of $10^{-10}$ mbar was achieved. For the regeneration cavity the operation of ion getter pumps is problematic as they are a potential source of light from glow discharges in the pumps. Therefore they will be switched off during the measurements searching for hidden photons. However, the pressure in the pipe can be easily maintained at about $10^{-6}$ mbar by turbo-molecular pump-stations.

\subsubsection{The vacuum system for the optical resonators of ALPS-IIc}

Three vacuum vessels at room temperature contain the optical elements of the experiment at the ends of the magnet strings and in the middle between the strings (see Fig. 36). In each vessel the breadboard for the optical elements is supported from the optical table at three points. The supports are mechanically decoupled from the vacuum vessels by soft bellows (see Fig. 36). The vessels will be supported from the floor of the cleanrooms. This concept will be tested at ALPS-IIa.

The vessels will be separable from the vacuum in the magnet strings by all-metal gate valves, to allow venting of the vessels for work on the optical components, while the magnet strings are at liquid Helium temperature. If the work on the optical components in the vented vessel requires transmission of the laser light through both production and regeneration cavity, i.e., an opening of the gate valves, a complete warm up of the magnet strings will be necessary. Flanges on the vessels with electrical feed-throughs will allow the connection of electro-optical elements and sensors. A flange in the central vessel will allow the operation of the "light tight wall" shutter. 

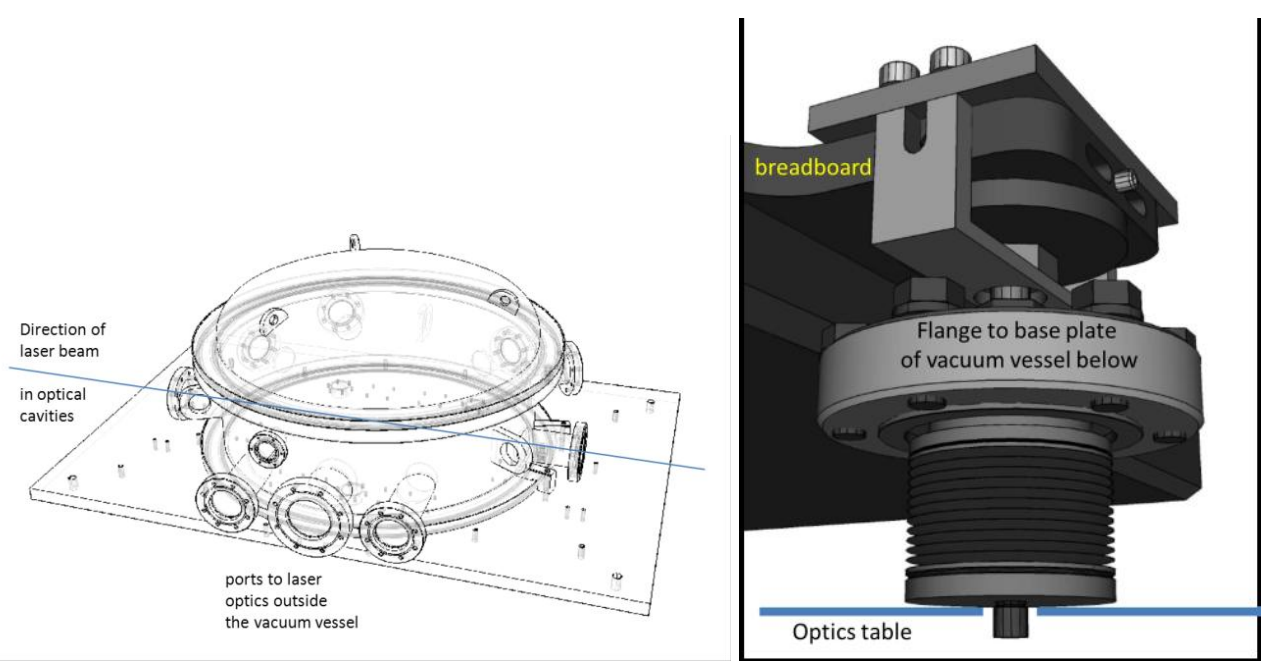

Figure 36. Vacuum vessel for the optical elements in the middle of the experimental setup and one support of the optical breadboard.
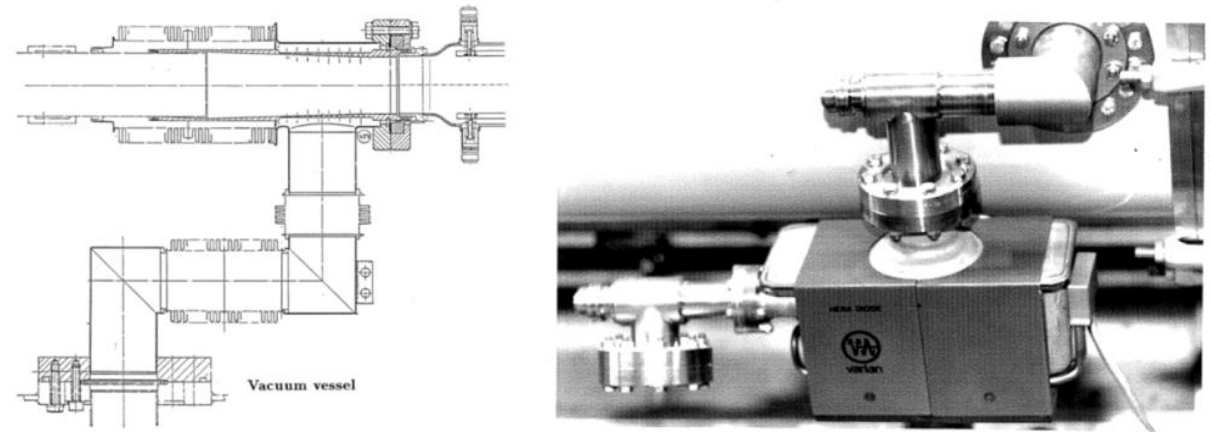

Figure 37. Pumping port (left) and pumping port with Ion Getter Pump (right).

The large flanges at the top and at the bottom of the vessels will be sealed by O-rings to ease the access to the optical elements in the vessels. To reduce the permeation through the O-rings there will be two rings per flange spaced a few $\mathrm{mm}$ apart. The space between the O-rings will be pumped to about 1 mbar by a small membrane pump. This concept allows to obtain pressures below $10^{-8}$ mbar in the vessels.

The vessels will be pumped by oil-free turbo molecular pump stations and NEG pumps. The use of NEG pumps which pump only chemically active gases, will allow the insertion of Helium gas into the vacuum system (see below), while maintaining a low partial pressure of active gases.

Each string of 10 superconducting HERA dipoles will be containing 2 dipoles with a pumping port (see Fig. 37), one being connected via a manual valve to an Ion getter pump of 60 liters per second [118], the other to a rupture disk, which serves as an overpressure valve (see Fig. 38). For pump down from atmospheric pressure to $<10^{-5}$ mbar, a pump station with a turbo molecular pump will be connected (see Fig. 39). Once the beam tubes in the magnet strings approach liquid Helium temperature external pumps are not needed anymore as the wall of the tubes acts as a cryopump, and the external pumps will be disconnected by the manual valves. The pressure in the cold 

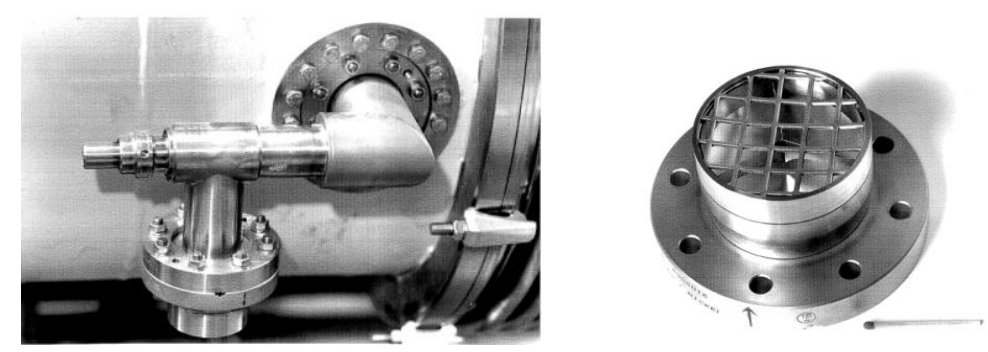

Figure 38. Pumping port with rupture disk.

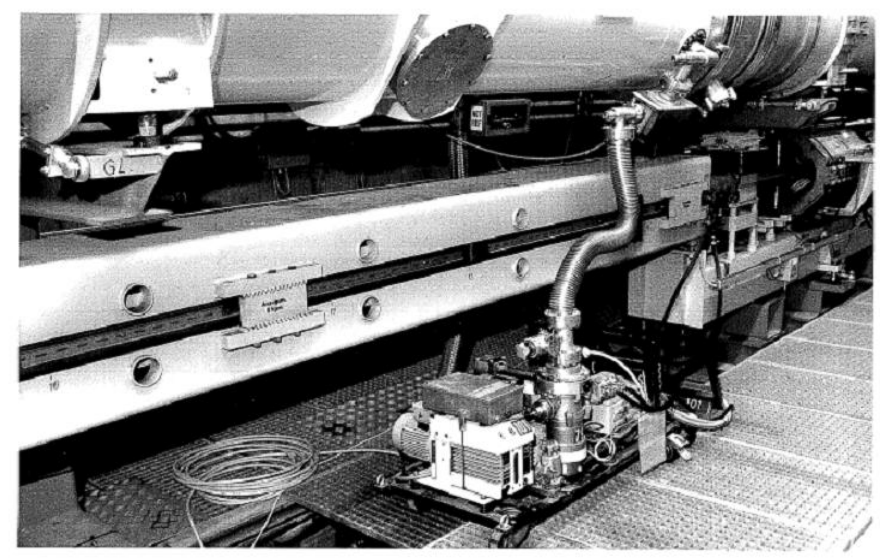

Figure 39. Turbo-molecular pump station.

sections will be well below $10^{-10}$ mbar.

The procedures for assembly and leak checks will be the same as for HERA [119].

\subsubsection{The insulation vacuum system}

At HERA the insulation vacuum for the chain of superconducting magnets was segmented into sections by vacuum barriers in the quadrupole cryostats [119]. At ALPS-IIc there are no vacuum barriers, so the two $100 \mathrm{~m}$ long strings of cryostats, connected by the kicker bypass line (see Sect. 3.5.3), have a common insulating vacuum. This will not pose any problem, as demonstrated at HERA, where during operation the vacuum barriers were bridged by vacuum bypass lines, forming one $\approx 600 \mathrm{~m}$ long insulation vacuum section. The insulation vacuum of the magnet strings will be continuously pumped by a few turbo molecular pump stations as at HERA to a pressure of about $10^{-6}$ mbar.

The procedures for assembly and leak checks will be the same as for HERA [118].

\subsubsection{Increasing the refractive index in the optical resonators of ALPS-IIc}

To improve the range of ALPS-IIc concerning the coupling constant and the mass of the searched for ALPs, the momentum transfer $q$ between the laser photon and the axion-like particle has to be varied, cf. Sect. 2.2.1. This can be achieved by a change of the refractive index $n$ in the beam pipe for both the production and the regeneration side of the experiment. The momentum transfer is 


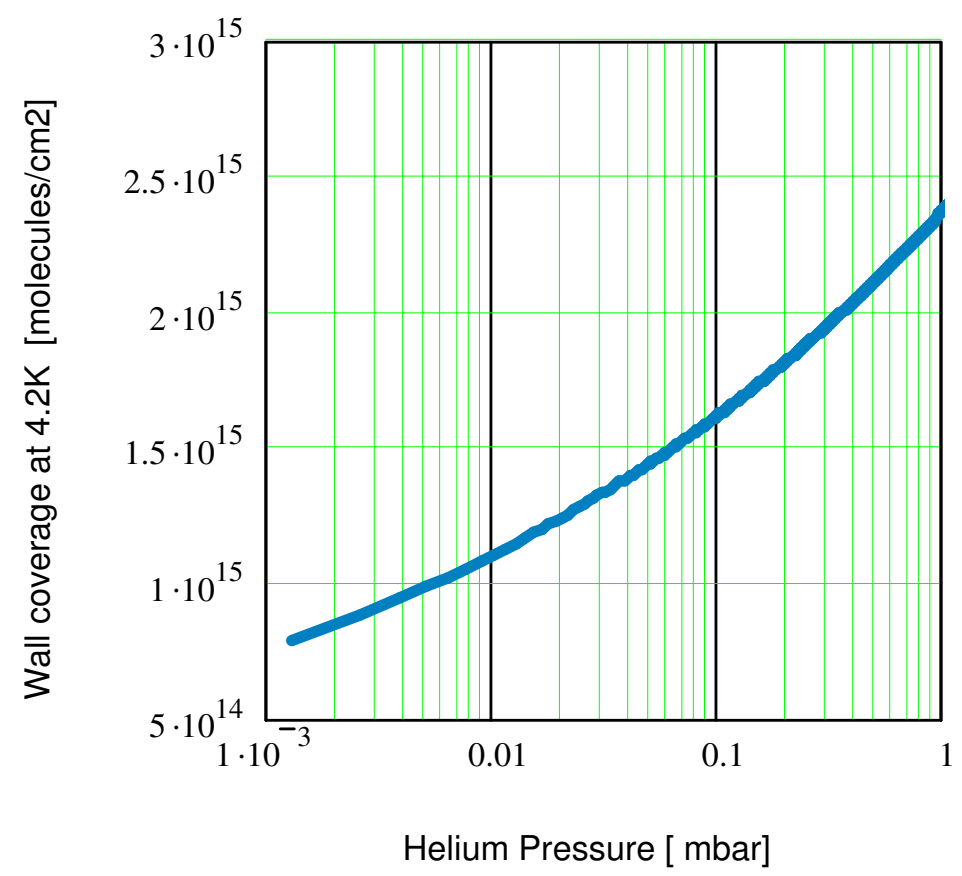

Figure 40. Wall coverage in $\frac{\text { molecules }}{\mathrm{cm}^{2}}$ as a function of pressure. The scaling formula is taken from E. Wallen [120].

given by:

$$
q=n \cdot \omega-\sqrt{\omega^{2}-m^{2}}
$$

where $\omega$ is the photon energy and $m$ is the mass of the axion-like particle.

In ALPS-I [6], the refractive index was changed by injecting Argon gas into the vacuum pipe. This procedure is applicable also to ALPS-IIb. However, as the wall of the vacuum pipe at ALPSIIc is at liquid Helium temperature, in contrast to the experimental setup of ALPS-I, gases will condense on the cold surface with too low a vapor pressure to be of any use.

Only the insertion of Helium gas can lead to a pressure increase in the pipe, yielding adequate values for the refractive index. Helium atoms will also condensate on the wall of the pipe, but as soon as the coverage exceeds a monolayer $\left(\approx 10^{15} \frac{\text { molecules }}{\mathrm{cm}^{2}}\right)$ the gas pressure in the pipe at liquid Helium temperature will reach values (see Fig. 40), which lead to a sufficiently large increase of the refractive index and thus the momentum transfer $q$.

The pressure shown in Fig. 40 is measured at room temperature, for example at the pumping port shown in Fig. 37. The pressure $p_{\mathrm{w}}$ at room temperature $\left(T_{\mathrm{W}}\right)$ and the pressure $p_{\mathrm{c}}$ in the cold pipe $\left(T_{\mathrm{c}}\right)$ are connected by the Knudsen relation

$$
\frac{p_{\mathrm{c}}}{p_{\mathrm{w}}}=\sqrt{\frac{T_{\mathrm{c}}}{T_{\mathrm{W}}}} .
$$




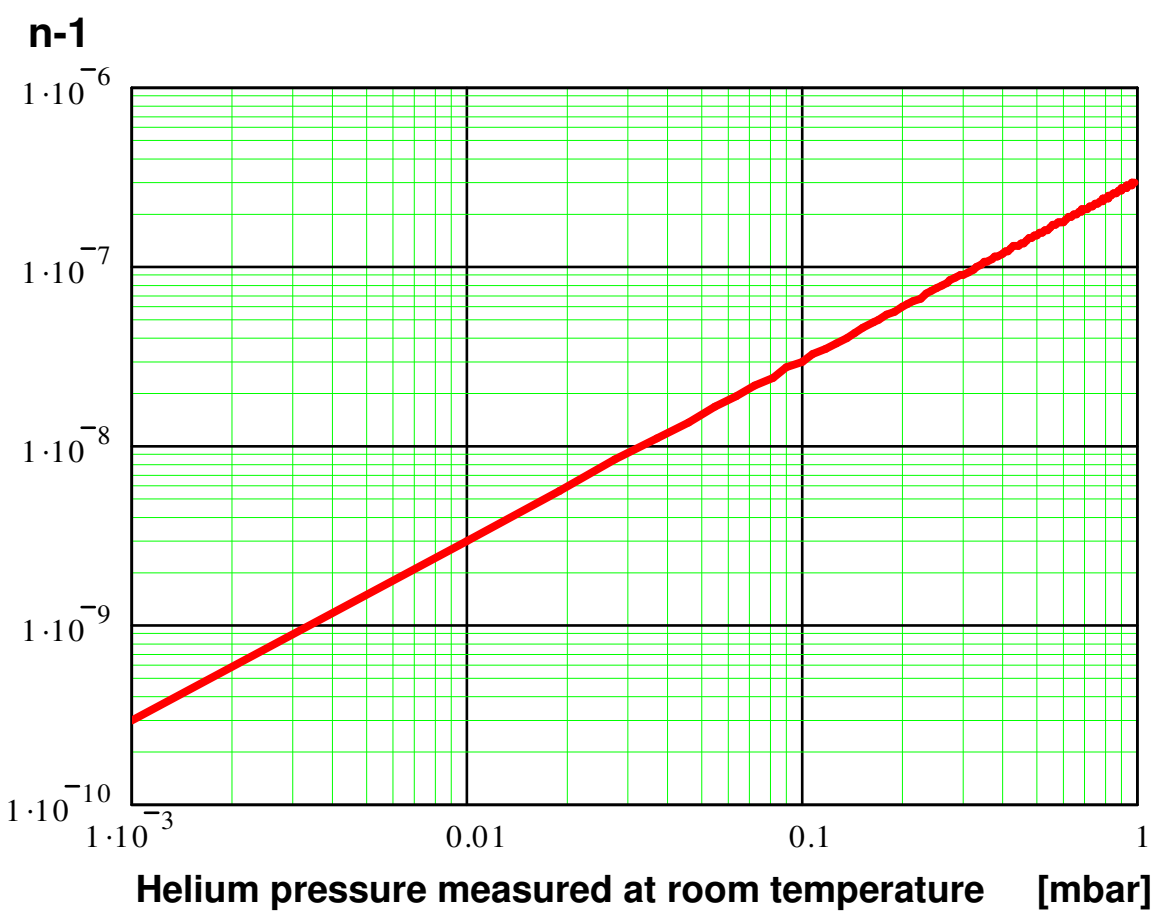

Figure 41. Relation between Helium pressure measured at room temperature and deviation from vacuum refractive index $n-1$.

Using this relation and the Lorentz-Lorenz law, one can determine the refractive index [121] in the cold pipe from the pressure $p_{\mathrm{w}}$ measured at room temperature (see Fig. 41), through

$$
(n-1)=\sqrt{\frac{T_{\mathrm{w}}}{T_{\mathrm{c}}}}\left(n_{\mathrm{w}}-1\right) \frac{p_{\mathrm{w}}}{1000 \mathrm{mbar}},
$$

where $T_{\mathrm{w}}=300 \mathrm{~K}, T_{\mathrm{c}}=4 \mathrm{~K}$ and $n_{\mathrm{w}}$ the refractive index at room temperature and $1000 \mathrm{mbar}$.

The production probability for axion-like particles [57] (see Eq. 2.1) does essentially not change up to a pressure (at room temperature) of $\approx 0.001$ mbar for the chosen photon wavelength of $1064 \mathrm{~nm}$. Fig. 42 shows the spiky structure of the probability for a pressure of $10^{-3}$ mbar. Varying the pressure to higher values (up to $\approx 0.5$ mbar) will shift the position of the spikes and thus leading to a smooth coverage of the ALP's mass range.

A pressure stability of 0.001 mbar will keep the conversion probability in the production and regeneration regions stable at a level better than $99 \%$. This will be feasible by measuring the pressure with capacity manometers, as the partial pressure of Helium is dominant in the range of interest, compared to other gases in the warm part of the vacuum system.

To establish a certain Helium pressure in the cold pipe, Helium gas will be injected into the vessels at room temperature at the ends of the magnet strings. Helium atoms will condensate first on the wall of the cold pipe close to the point of injection. Then the wall coverage will slowly propagate along the pipe, eventually reaching the far end of the pipe [120]. In the pressure range of interest for ALPS-IIc it will take a few hours to reach a homogeneous and stable coverage, indicated by equal Helium pressure in the vacuum vessels at room temperature. 


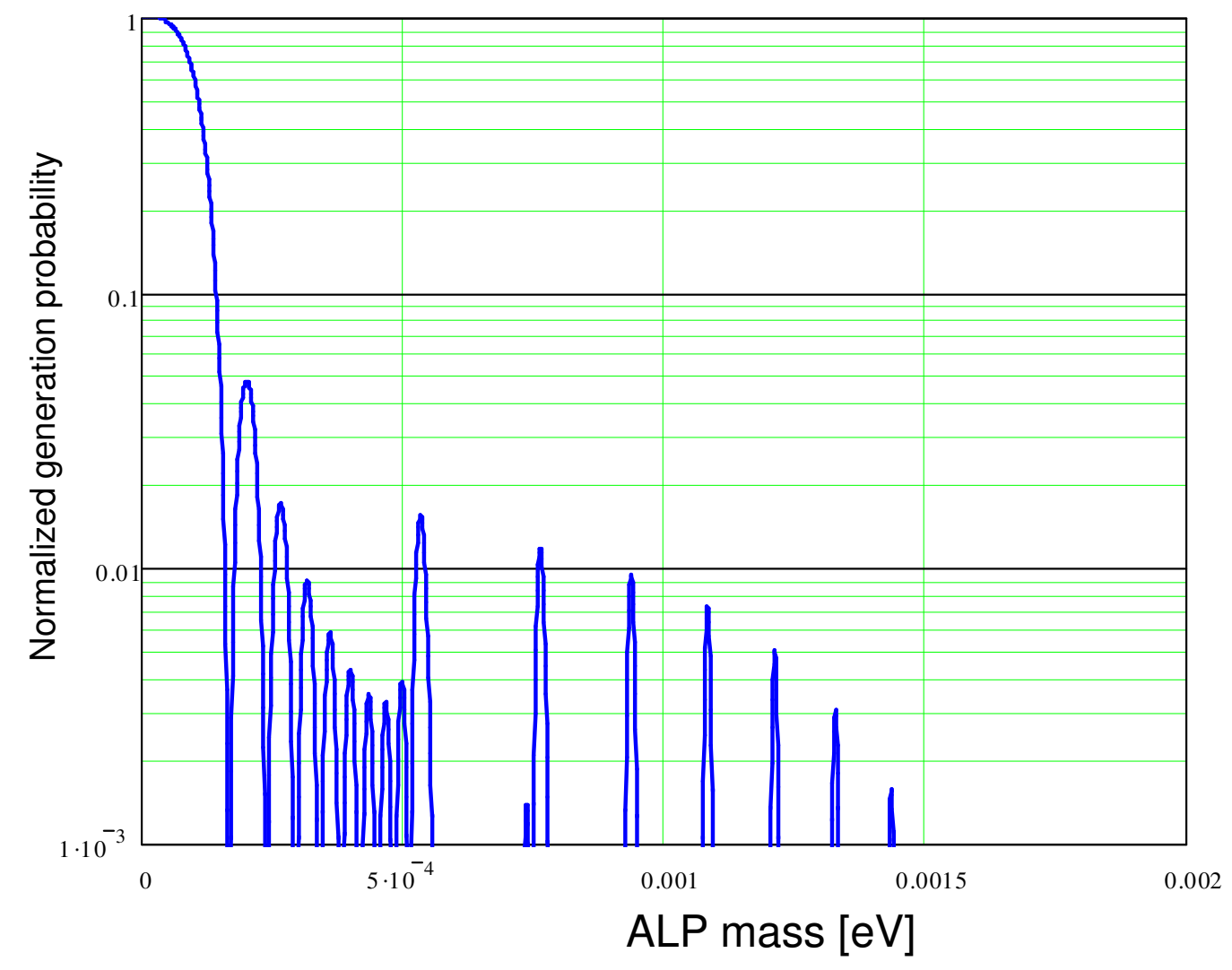

Figure 42. Normalized production probability at $10^{-3}$ mbar as a function of ALP mass.

For the variation of the momentum transfer $q$ the Helium pressure will be changed upwards from 0.001 mbar to $0.5 \mathrm{mbar}$, as the removal of Helium from the walls of the cold pipe will require a warm up of the magnet strings well above $4.2 \mathrm{~K}$.

The round trip losses in the optical cavities due to Rayleigh scattering off Helium atoms will be very small $\left(2 \cdot 10^{-8}\right.$ at 0.5 mbar), thus not leading to a reduction of the power buildup of the cavities. The additional thermal load on the magnet strings due the scattered photons is negligible $(\approx 0.01$ Watt $)$.

\subsection{Data acquisition}

The data acquisition system (in the following DAQ) will provide the front-end to the detector readout and various subsystems (including the slow-control). The front-end will include control over various subsystems and will record monitoring informations during the operation of the experiment in data-taking and calibration modes. The various data-streams from the detector and the monitoring systems will be collected and stored for later analyses.

Technical challenges The DAQ has to be scalable to the different stages of the ALPS-II experiment and to be flexible for changes in the photon detector during the different setups. The amount of data to be read-out and handled is modest and within the performance of available technology. 
We are expecting event rates well below $\mathrm{kHz}$ in triggered mode (mostly during calibration runs) for the TES and CCD read-out rates probably well below a $\mathrm{Hz}$ for calibration and even smaller for data-taking.

Conceptual design The suggested design of the TES-readout is based on a commercially developed digitizing system (ATS9462 from AlazarTech [122]) for the actual data taking. The digitizing board allows to sample and digitize up to 512 MSamples with a frequency of $180 \mathrm{MSamples} / \mathrm{s}$ and 16 Bit dynamic range. This PCIe based board has been in use for the digitization of TES signals at the group 7.21 at the Physikalisch Technisches Institut in Berlin. For the initial setup and tests an already available DRS-4 board of the ETH Zürich with 4 channels in self-triggering mode can be used [123] as well. In case of a CCD-camera as detector system, the camera can be connected directly via a USB interface to the read-out PC. The node (detector and read-out) handles the communication and transfer of data to a server system. There, the data is collected and stored in a relational data-base system (preferentially MySQL). The inter-process communication will be based upon a state-machine system which defines states (e.g., inactive, configuring, ready) and allowed transitions between the states. The unified state-machine approach will provide welldefined access and control over the various sub-systems. The actual protocol for the inter-process communication and control is based upon XML (e.g., XML-RPC [124]) (see Fig. 43 for a scheme of the DAQ system).

In a similar way, the interface to additional slow-control systems will be incorporated including

1. Magnet control: The superconducting magnets will be controlled by the cryogroup at DESY. The control environment is based on the EPICS (Experimental Physics and Industrial Control System), which is in wide use at accelerator facilities. The ALPS-I experiment used a thin client which would communicate via the http protocol to the actual user. A similar scheme will be used, extending the HTML syntax to XML. The parameters passed to the DAQ will include the electrical current, the temperature, as well as error messages in order to diagnose a quench.

2. Safety loop for the laser: The safety loop will monitor the status of the doors which connect the grey room from the outside, the emergency switch-off buttons, as well as the integrity of the laser protection covers (personal interlock). The technical interlock will interrupt the operation if the room temperature exceeds a defined limit, the pressure in the pipes is too large (vacuum system), or the superconducting magnet quenches. Additional warnings will be provided if any other system is in a non-ready state.

For safety reasons, any breaches of the interlock system will have to lead to an immediate shutdown of the laser system.

The temperature switch can be implemented by using the Uniflex CI45 module from PMA Prozess- und Maschinen-Automation [125]. These modules need a $24 \mathrm{~V}$ power supply and are mounted on an industrial standard (DIN) rail (Hutschiene). The measured temperature can be read out directly by the module. The modules can be programmed so that a relay switches at an upper or lower limit. This relay can be connected directly with the safety loop. The combination of ARM based computer nodes (e.g., Plug-PC or RASPBERRY PI) which act as interfaces to microcontroller units (e.g., Arduino boards: these boards provide 14 D/A ports and 6 A/D ports, access 


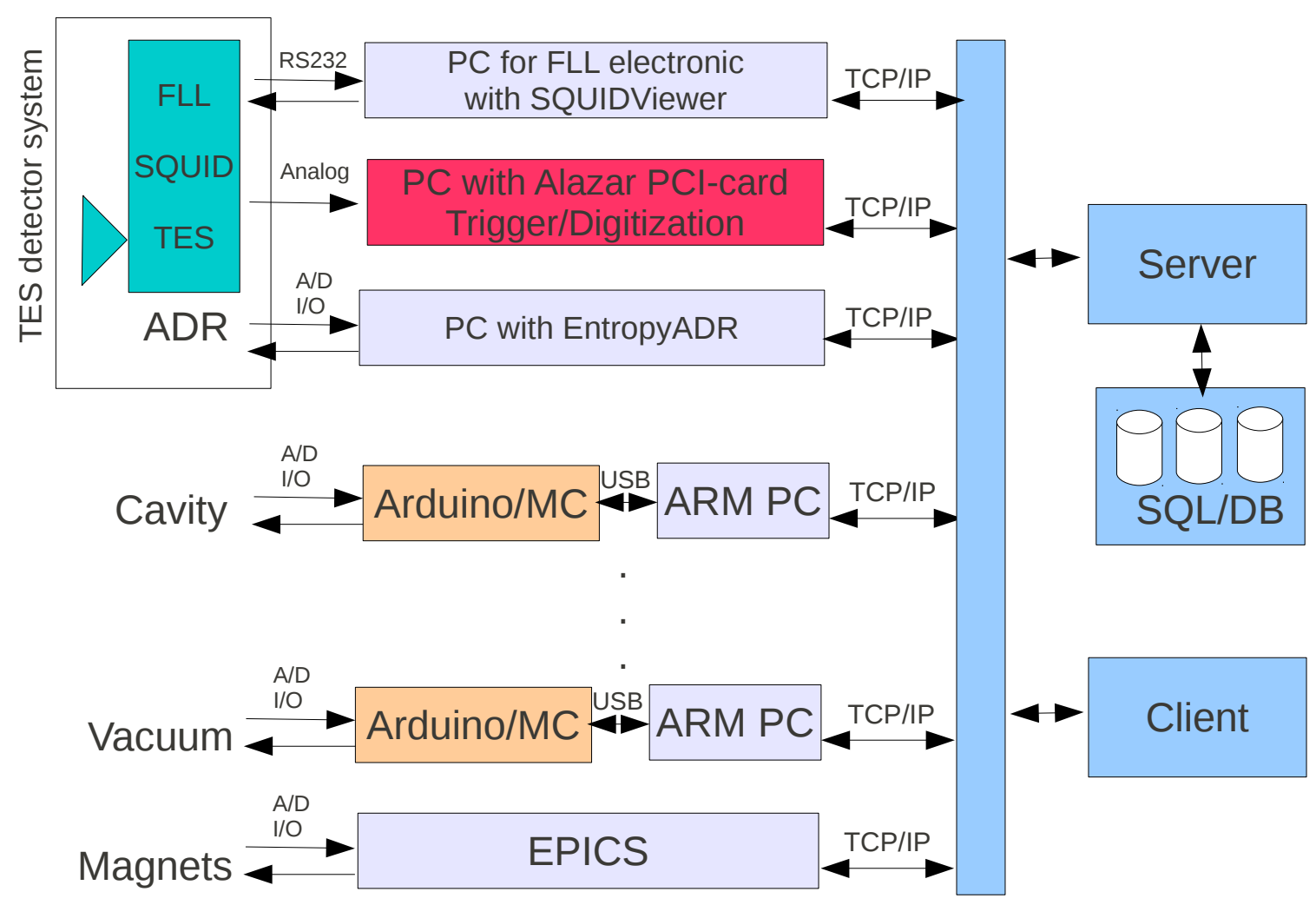

Figure 43. Scheme of the distributed data acquisition and control system.

through a USB interface). These ready-to-use micro-controller boards are a convenient solution based upon commercially available hardware that is low-priced and very flexible.

Again, the individual sub-systems will be logically accessed as a state-machine from the server. Data-streams will be stored in a data-base. The actual number of monitoring systems is either limited by the address space of the TCP/IP stack (unlikely to be relevant) or the required bandwidth that can be handled by the server which is used for communication. For the initial setup, tens of monitoring channels distributed over five nodes will be used.

The front-end to the user will be available through a client program that can be run on any computer within the same sub-domain of address space. At a later stage, remote-operation through secure network protocols will be implemented (at a lower priority). The client will have control over the type of data-taking performed (regular data taking and calibration data taking), will be able to set parameters of the data-taking (e.g., exposure times of the CCD, triggering threshold for the TES) and access and control all sub-systems. The parameters will be stored in the data-base in order to recover the state of the entire system at any time.

The actual data retrieval for analysis will be handled through access of the MySQL data-base. A number of simple client scripts allow to select and retrieve data and calibration information preferentially in FITS-type files.

Expected performance The required performance of the system is well-matched with the proposed system. In case of a TES detector system, the most challenging component is the read-out 
of the data during calibration runs. In this particular case, it may be necessary to digitize with a high sampling frequency in the ideal case continuously the signal for a fraction of a second. During regular data-taking, the expected background rate of the TES will be small $\left(10^{-4} \mathrm{~Hz}\right)$ and will be of no concern. The proposed PCIe-based digitizer system will allow both continuous data-taking for a few seconds to monitor the base-line accurately as well as triggered operation with additional on-board digital pulse-shaping (if needed).

The other systems will have modest read-out rates below kHz. Thus, the traffic of tens of nodes can be handled easily with regular network bandwidth.

\subsection{Site considerations for ALPS-IIb and IIc}

\section{ALPS-IIb}

For ALPS-IIb two optical cavities of about $100 \mathrm{~m}$ length will be set up with very little infrastructure effort in the straight section HERA West. The still existing vacuum pipe of the HERA proton ring can be used for ALPS-IIb as it is straight over about $2 \cdot 160 \mathrm{~m}$ in contrast to the other straight sections of HERA, where vertical bending magnets limit the available straight length in the vacuum pipe to about $2.60 \mathrm{~m}$. The aperture of the proton beam pipe (see Fig. 44) is large - except for one location which can be easily modified - and allows the operation of optical cavities as described before.

Locations in hall West and in the tunnel on both sides of the hall have been identified (see Fig. (45), where little effort is required to remove the existing accelerator installation to free space for the clean rooms at an adequate distance from the hall.

\section{ALPS-IIc}

For ALPS-IIc the best possible setup - allowing for a power buildup of 40000 in the optical cavity on the regeneration side of the experiment- consists of $2 \cdot 12$ straight HERA dipoles. With the overall dipole length of $9.766 \mathrm{~m}$ such a setup requires a total length of about $250 \mathrm{~m}$ including the space for the cleanrooms and laser huts. The base line design for ALPS-IIc with $2 \cdot 10$ almost straight dipoles requires a total length of about $210 \mathrm{~m}$.
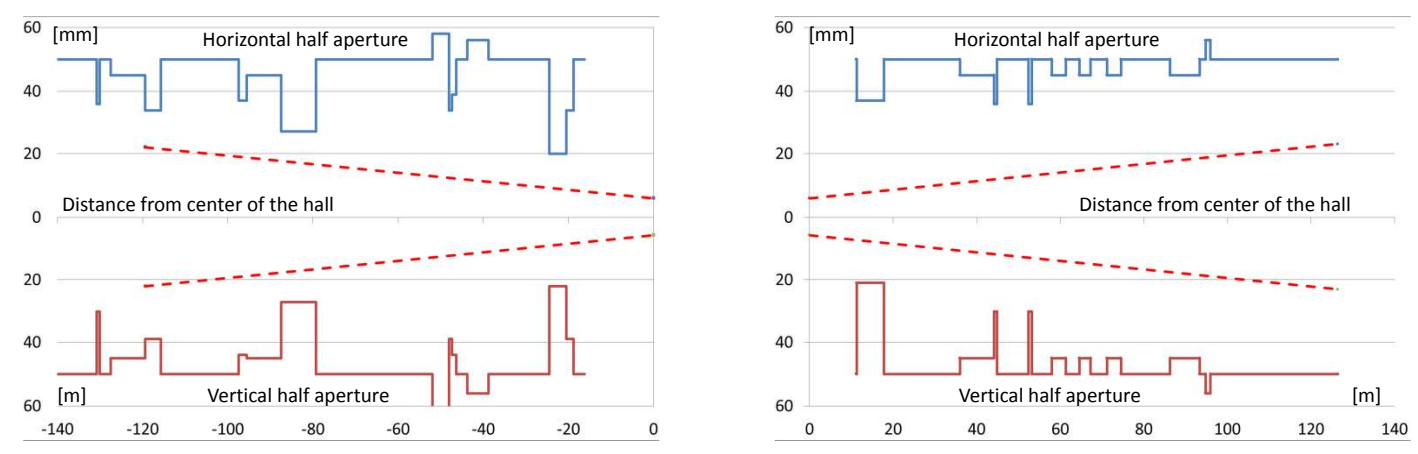

Figure 44. The figure shows the horizontal and vertical apertures of the proton beam pipe to both sides of HERA hall west. The Gaussian laser beam with a size required for a power buildup of 40000 is sketched as a red dotted line. As can be seen there is ample space to accommodate the laser beam on both sides. 
The natural choice for the setup of dipole strings with these lengths is a straight section of the HERA tunnel due to the principal availability of infrastructure like cryogenics. The available length in the long straight sections East and West amounts to $320 \mathrm{~m}$, long enough for a setup of $2 \cdot 12$ HERA dipoles for ALPS-IIc. The short straight sections South and North with a length of $220 \mathrm{~m}$ allows the setup of $2 \cdot 10$ HERA dipoles.

An installation outside of the HERA tunnel on the DESY site has also been considered. There are possible locations with sufficient length along the eastern borderline of the DESY site. Due to the additional costs of such an installation for buildings, cryogenics, and infrastructure, which have been estimated to about 12 million Euros, this possibility has not been followed any further.

The installation of the dipole strings connected properly to cryogenic boxes requires the removal of the existing accelerator installation in any straight section of HERA chosen for this purpose. This is mainly due to the necessary exchange of the cryogenic boxes from one end of the straight section to the other, to match the connection pattern of the dipoles. The effort in this respect is largest in the straight section West due to the large number of special and complicated accelerator systems like the proton injection line, the superconducting cavities of the electron ring, the RF cavities of the proton ring, and the proton beam dump. The beam dump would have to be disposed of [126], which would cost a few million Euros.

The setup of HERA dipole strings in the straight section East would require the highest operation cost for the cryogenics of all straight sections. In addition the power supply for the superconducting magnet chain of HERA would have to be moved from hall West to East. However, the straight section East remains the location for a setup of $2 \cdot 12$ dipoles string with lower cost as compared to West, due to the disposal cost for the beam dump.

For the base line design of the ALPS-IIc experiment with $2 \cdot 10$ dipoles the total cost is lowest

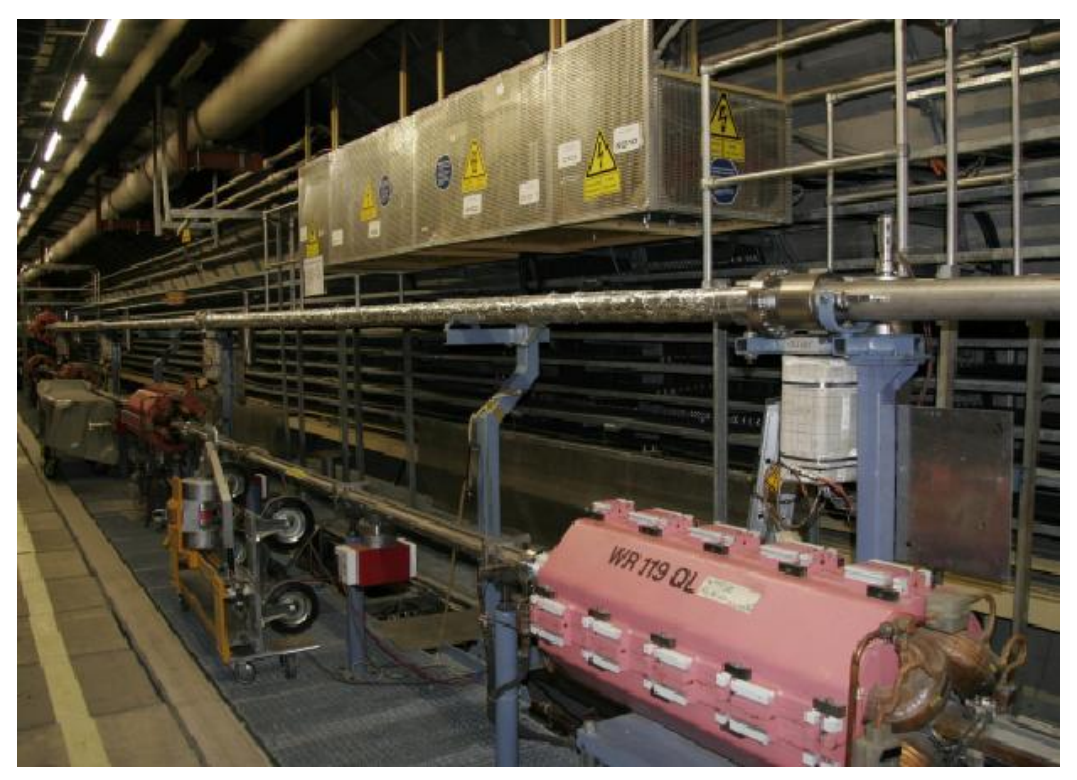

Figure 45. This picture shows a part of the HERA West right section. Here, there are about $25 \mathrm{~m}$ space for an optical setup requiring little effort for disassembly. 
in the straight section HERA North, which therefore has been selected as best suited for the setup of ALPS-IIc.

\subsection{Setup and installation}

\section{ALPS-IIb}

In the straight section HERA West the infrastructure for two optical cavities of about 100m length will be set up with very little infrastructure effort. The existing vacuum pipe of the HERA proton ring can be used for ALPS-IIb as it is straight over about $2 \cdot 160 \mathrm{~m}$ in contrast to the other straight sections of HERA, where vertical bending magnets limit the available straight length in the vacuum pipe to about $2 \cdot 60 \mathrm{~m}$.

Locations in hall West and in the tunnel on both sides of the hall have been identified, where only minor activities are required to remove the existing accelerator installation to free space for the clean rooms at an adequate distance from the hall.

\section{3}

The accelerator installation at the locations for the cleanrooms can be dismantled by the DESY group MEA, whenever manpower is available throughout the whole year, creating space for the installation of the cleanrooms. The construction of the laser huts will be designed and orders for the construction as well as for the clean room equipment will be placed.

\section{4}

The cleanrooms will be installed within the first half of the year. With the completion of the ALPSIIa experiment in the middle of 2014 (see Sect. 3.2) the optical components and the optical tables become available and will be installed in the cleanrooms.

\section{5}

The $\sim 100 \mathrm{~m}$ long production and regeneration cavities will be setup and commissioned, allowing physics runs in the second half of the year.

\section{ALPS-IIc}

\section{5}

In the beginning of 2015, with the completion of the XFEL project, manpower will be available at DESY to install the ALPS-IIc setup and also cryogenics capacity, allowing the cryogenic tests of the HERA dipoles for ALPS-IIc on the magnet test bench.

In parallel to the magnet tests, the accelerator installations in the straight section North will be disassembled. For the disassembly of the normal conducting magnets and cavities in the straight section the "HERA tram" (see left side of Fig. 46) will be used, which has been transfered from hall West after the removal of the Kicker bypass from the HERA tunnel.

After the deinstallation of all elements in the straight section, the cryogenic boxes at the ends of the straight section will be disconnected and moved to the opposite sides.

Then activities will follow, which are easier without the magnet strings in place, like the modification of the quench gas collection pipe, modification of dump resistors, connections for cooling water of the laser huts, or work on the main power cables. 

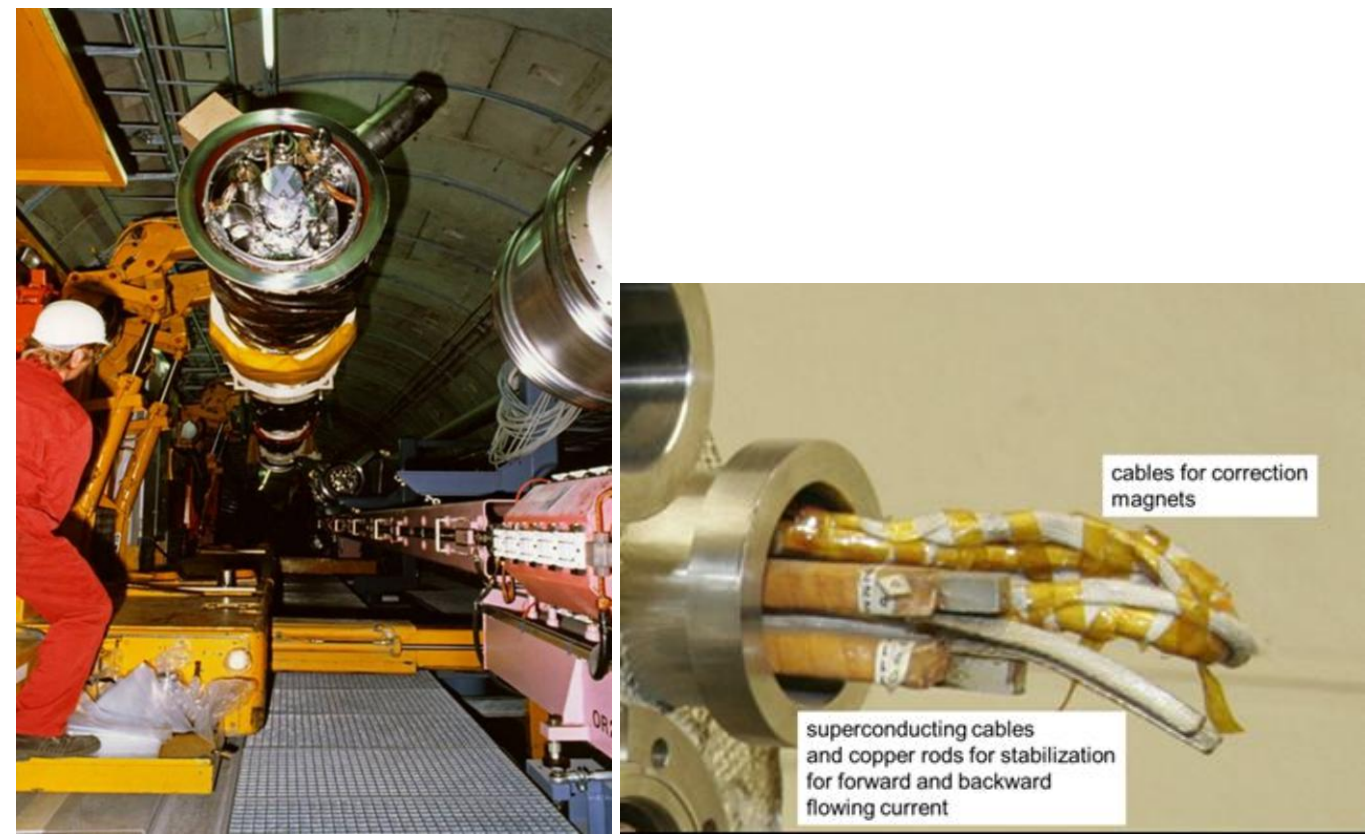

Figure 46. HERA tram (left) and superconducting cables in $4 \mathrm{~K}$ Helium tube (right).

\section{6}

New supports for the dipoles will be installed. For the installation of the dipoles the "HERA tram" will be used. In general the installation of the dipole strings will follow the procedures established for the installation of the superconducting magnets at HERA [127].

After the installation of the superconducting dipoles, the HERA kicker-bypass will be installed between the two strings. To compensate the force of about $80 \mathrm{kN}$ by the atmospheric pressure on the end flanges of the kicker-bypass, the endflanges are connected by 3 tension rods, which have to be replaced by a steel girder construction to allow later after the installation of the cleanroom access to the optical elements in the vacuum vessel.

The proper electrical connection of the superconducting cables for forward and return current between adjacent dipoles (see right side of Fig. 46) is of uttermost importance for the operation of the magnet strings. The heat generated by the resistivity of the connection must be small enough to be absorbed by the surrounding liquid Helium, to keep the temperature of the connection stable. For HERA a special tool was used to braze the connections properly by well-trained people [128]. Most of those people are in retirement by now, but a few are still at DESY, who will be capable of connecting the cables after some retraining. Video material ${ }^{28}$ is available which shows the procedures. The video material also shows how to insulate and to fix the cables after brazing to avoid an electrical discharge. Once the electrical connections are made and tested, the Helium pipes of adjacent dipoles will be joined, using special welding tools from HERA, shown in the available video material. These tools are still available and also knowledgeable people to weld the connections.

The electronics for the quench detection and protection will be placed in the tunnel close to

\footnotetext{
${ }^{28}$ Video material by Otto Peters retired from the DESY group MKS.
} 
the magnets after the completion of the magnet installation.

The laser huts $\left(2.25 \cdot \mathrm{m}^{2}\right)$ with the optical tables at the end of the strings and in the middle will be installed, using the available material from the completed ALPS-IIb experiment for the setup of the optical resonators and the detector. The cooldown of the magnet strings will be done once the commissioning of the optics will be completed, as venting of the vacuum pipe in the magnets for the commissioning of the optics can only be done when the magnets are at room temperature.

\section{7}

After the commissioning of the optical resonators, the detector and commissioning of the magnet string physics runs will start.

\subsection{Operation and run procedure}

As discussed in Sect. 2, some parameters will be varied over the course of the experiment in order to interpret a potential signal and distinguish the different types of WISP, and to fill the sensitivity gaps (cf. Figs. [ ] and [). These parameters include the magnetic field strength, the orientation of the laser polarization w.r.t. the magnetic field, and the pressure of the rest gas. The data taken with one set of these parameters will result in a run. During each run, the experiment will operate in different modes, which are dedicated either to assert the proper condition of the experiment or to take data. These are described below. Depending on the actual performance of the subsystems of the experiment and during the commissioning phase, additional modes may be necessary. A typical run is shown in Fig. 47 .

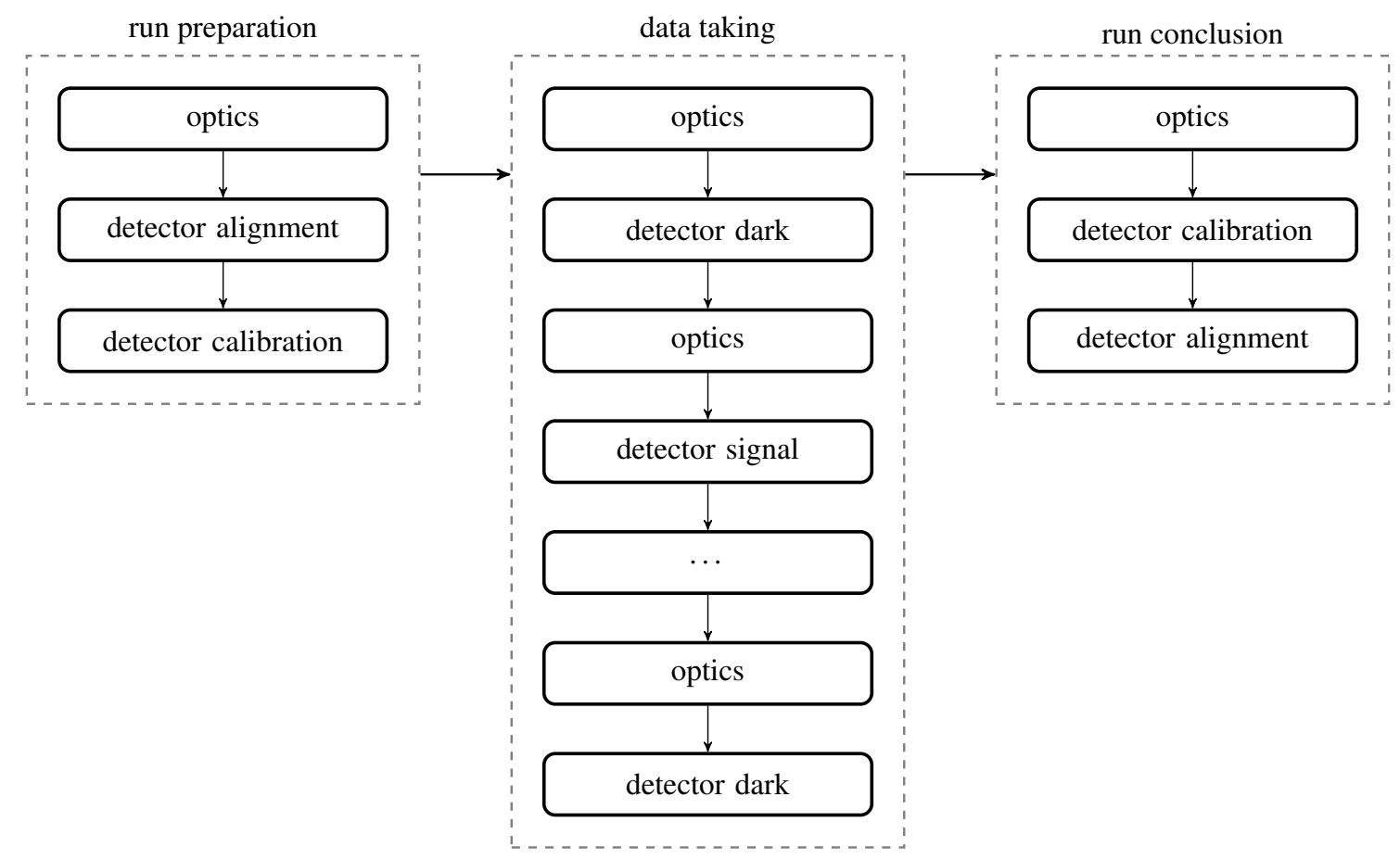

Figure 47. A typical run of ALPS-II in routine operation. 


\section{Condition Assertion}

Optics Mode During this mode, the optics system is brought up. This includes checking the alignment of the production and regeneration cavities, locking the two cavities and setting the resonance condition of the regeneration cavity for $1064 \mathrm{~nm}$ as necessary for the targeted data type (see below). The resonance condition for $1064 \mathrm{~nm}$ can be checked by monitoring QPD7 and opening the shutter on the central breadboard (Fig. 12). If the shutter is open, infrared light enters the (resonant) regeneration cavity and is recorded by QPD7.

Because the shutter may be open in this mode, $1.7 \mathrm{~mW}$ of $1064 \mathrm{~nm}\left(10^{16}\right.$ photons per second $)$ will leave the regeneration cavity and potentially reach the detector. Hence, the detector has to be protected by, e.g., a flip mirror, which redirects the light to a beam dump.

Detector Alignment Mode In this mode the alignment of the detector to the beam of reconverted photons is verified. To this end, the shutter on the central breadboard will be open. Infrared light will enter the regeneration cavity, following exactly the path of potentially regenerated photons. As mentioned above, $1.7 \mathrm{~mW}$ of $1064 \mathrm{~nm}$ radiation will hit the detector. Hence, a dedicated filter will be necessary to protect the detector from any damage due to this intensive radiation as discussed in Sect. 3.4.2.

\section{Data Taking}

It is foreseen to routinely operate the CCD and the TES in three distinct modes: calibration, signal, and dark. Signal and dark mode will only happen during stable operation of the cavities. They differ only in the resonance condition of the regeneration cavity. In signal mode the regeneration cavity will be resonant for infrared light while in dark mode it will be resonant only for $532 \mathrm{~nm}$. This will be achieved with the AOM on the central breadboard (Fig. 12). Thus, the regeneration probability is inhibited by a factor of the power build-up of the regeneration cavity for infrared light $(\sim 40000)$. This makes it possible to record all experimental background (e.g., stray light, fluorescence effects).

CCD Operation The calibration of the CCD will include the read-out of a bias-frame, a darkframe, and a flat-field, which will be used to assure a stable operation of the CCD. The expected stability during routine operation will reduce the need to take calibration data very frequently. However, complete calibration data will be taken before and after operation in signal or dark mode.

Regular data-taking in signal or dark mode will consist of a series of frames. The exposure time will be chosen such that the read-out noise and dark current will balance, which results in roughly one hour (cf. Sect. 3.4.3).

TES Operation The specific calibration and data-taking routine for the TES based read-out is at this point less-well defined as for the CCD. Generically, a calibration mode will be required to calibrate and characterize the detector, the SQUID amplification, and the digitization. All components are potentially a source of background and require independent calibration to characterize the baseline and its fluctuations. Currently, we envisage to use a triggered digitization with up to $180 \mathrm{MSample} / \mathrm{s}$ frequency. A continuous read-out of up to $512 \mathrm{MSamples}$ can be stored on-board, for longer data-taking, direct memory access is possible to store longer data-streams. This will however be only an option in exceptional cases. In the case of the DRS4-board, the maximum 
bandwidth of the read-out limits the read-out rate to a few hundred $\mathrm{Hz}$ (based upon the experience with the DRS4 board, the maximum rate is $530 \mathrm{~Hz}$ ). With a random trigger of the read-out, the baseline can be sampled in an unbiased way. However, any irregularity at frequencies smaller than $\mathrm{kHz}$ will not be visible. This is in principle not an issue given that the signals are much faster.

Dedicated calibration runs will be used to read-out pulses registered with the TES illuminated by light. The pulses will be analyzed to accumulate a photon number spectrum to calibrate the single-photon response and to set the trigger threshold.

During regular data-taking, the triggered signals are recorded (without any on-line pulseshaping) in a time window of $100 \mathrm{~ms}$ in order to cover the pulse as well as the base-line before and after the pulse. The trigger time is accurate to $8 \mathrm{~ns}$ and will be stored for each triggered event.

\subsection{Data analysis}

The analysis of the photon detector data will result in the number of regenerated photons, which has to be combined with other experimental parameters to determine the coupling strength to photons and possibly also the mass of a WISP. More accurately, a confidence interval will be estimated, yielding either an upper limit or an interval for the number of regenerated photons. Because the TES and the CCD deliver different types of measurement (the TES is a true single-photon detector while the CCD integrates the incoming photon flux during exposure), the procedure to estimate the number of regenerated photons has to by tailored for each detector.

The method to estimate the rate of reconverted photons for the two detectors is described below. The interpretation of a signal of re-converted photons is sketched in Sect. 3.1.2.

The signal recorded by any of the two detectors will be the sum of different components: the flux of reconverted photons, irreducible background, noise of the read-out, and a potential offset. The value of the signal, $v$, can be described by the following statistical model,

$$
v \sim \operatorname{Pois}\left(\lambda_{\text {sig }}\right) \oplus \operatorname{Pois}\left(\lambda_{\text {bgd }}\right) \oplus \operatorname{Gauss}\left(\delta, \sigma_{\text {ro }}\right) \oplus \text { Offset },
$$

where Pois $(\lambda)$ are Poisson-distributed components due to reconverted photons (sig) and background photons (bgd), Gauss $(\mu, \sigma)$ the component due to noise of the read-out (ro), which is assumed to be Gaussian, and Offset a constant. The measured value, $v$, is distributed like the convolution of the distributions of the components, which is represented by $\oplus$.

CCD The CCD was already used in ALPS-I and later tested in detail to determine its performance for $1064 \mathrm{~nm}$ photons. The analysis procedure is based on these experiences and, naturally, more matured than the approach for the TES, where the data analysis will depend on the results of the ongoing R\&D.

Interpreting the components of Eq. 3.4 in the case of the CCD is straight forward. The Poissonian background is due to the accumulation of thermally produced dark charge; the read-out noise is caused by the amplification and digitization during the read-out of the camera; and an offset will be present due to biasing the sensor chip. Pixels outside of the signal region on the camera can be described by the same model by setting $\lambda_{\text {sig }}$ to zero.

It was found that the bias level of the camera shows approximately linear, spatial variations similar for each frame (fixed pattern) and varies by a global offset on a per-frame basis. To correct this per-frame variation of the offset, the value of the signal pixel will be corrected by subtracting 
the average of a large number of pixels in the vicinity of the signal pixel. Because the variation of the bias is approximately linear, the spatial variation of the bias is averaged and can be dropped if these pixels are distributed symmetrically around the signal pixel. Thus, the corrected signal pixel value, $v_{\mathrm{c}}$, is described by

$$
v_{\mathrm{c}} \sim \operatorname{Pois}\left(\lambda_{\text {sig }}\right) \oplus \operatorname{Gauss}\left(\delta, \sigma_{\text {ro }+ \text { dc }}\right),
$$

where the Gaussian now contains the components of the read-out noise and dark current of the signal pixel as well as of the averaged pixels and incorporates a possible systematic offset, $\delta$, between these.

Any signal is searched for by comparing so-called data frames (data, where re-converted photons from WISP can be expected) with dark frames without any re-converted photons. Correspond-

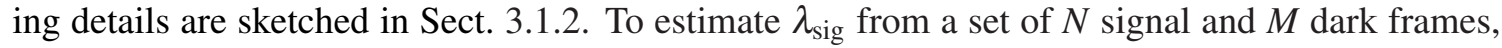
we will use the difference of the averages of the corrected pixel values, $t$,

$$
t=\frac{1}{N} \sum_{i=1}^{N} v_{\mathrm{c}}^{(\mathrm{sig})}(i)-\frac{1}{M} \sum_{j=1}^{M} v_{\mathrm{c}}^{(\mathrm{dark})}(j)
$$

which is described statistically by

$$
t \sim \frac{1}{N} \bigoplus_{i=1}^{N} \operatorname{Pois}\left(\lambda_{\text {sig }}\right) \oplus \operatorname{Gauss}\left(0, \sigma_{t}\right),
$$

where the first term represents the averaging of $N$ Poisson-distributed observables and $\sigma_{t}$ depends on the sizes of the signal and dark data sets, $N$ and $M$, and on the noise from the read-out and the dark current, $\sigma_{\mathrm{ro}+\mathrm{dc}}$,

$$
\sigma_{t}^{2}=\left(\frac{1}{N}+\frac{1}{M}\right) \sigma_{\mathrm{ro}+\mathrm{dc}}^{2}
$$

An algorithm based on [129] has already been implemented to estimate $\lambda_{\text {sig }}$ for an observed value of $t$, where $\sigma_{\mathrm{ro}+\mathrm{dc}}$ is estimated from the dark frames. Monte Carlo simulations showed that for $N>10$ and $M \geq 10 \cdot N$ this gives precise coverage. Systematic tests of this method will include using different groups of pixels for the offset correction and applying the method on non-signal pixels.

TES The TES provides single-photon informations which can be extracted from the pulse shape (e.g., rise-time, height, pulse-width). The pulse has a typical risetime of 300ns. With the proposed read-out system that samples at a frequency of $180 \mathrm{MSamples} / \mathrm{s}$, the rise-time of the pulse will be very well resolved with $\approx 50$ samples.

In the simplest scheme, a trigger threshold will be set to suppress the base-line noise and will trigger the read-out of the digitized pulse and the adjacent base-line ${ }^{29}$.

The trigger threshold will be regulated to suppress unwanted noise of the base-line while maintaining a high photon detection efficiency. The resulting background will be given by real photons (mostly thermal) $\left(\lambda_{\mathrm{bgd}}\right)$ as well as read-out noise $(\operatorname{Gauss}(\delta, \sigma))$. The analysis of the events

\footnotetext{
${ }^{29} \mathrm{~A}$ more refined triggering would be based upon the image shape and could be implemented in the FPGA of the digitizer
} 
can be used to suppress background photons which are not in the right wavelength band (pulseheight) or do not show the characteristic pulse-profile. The former will reduce the value of $\lambda_{\text {bgd }}$ while the latter will help to reduce the impact of base-line/readout. A possible statistical analysis of the TES data could in principle be carried out in a similar fashion as for the CCD. However, it may be more sensitive to develop an analysis which will benefit from the individual pulse measurements. Such an analysis would calculate a probability for each observed pulse to be produced from a reconverted photon, a background photon, or to originate from noise. In such an analysis, hypothesis testing and parameter estimates would be based upon a likelihood method.

\section{Summary \& concluding thoughts}

Low-energy particle physics experiments enable us to explore fundamental physics in a complementary way to accelerator-based searches by looking for new light particles with tiny couplings. Such new particles arise naturally in many extensions of the Standard Model and might also explain observations that are not accounted for within the particle physics known today (important examples being the absence of $\mathrm{CP}$ violation in the strong interactions and the nature of Dark Matter).

We have presented here the technical design for the proposed ALPS-II experiment at DESY which could contribute strongly to exploring the widely unchartered territory of Weakly Interacting Slim Particles (WISPs). ALPS-II would improve the best present-day laboratory sensitivity achieved by ALPS-I by more than three orders of magnitude and even surpass indirect limits on WISP properties from astrophysics and solar observations. Thus, parameter regions motivated by astroparticle physics phenomena and from predictions of string theory are in reach of ALPS-II.

ALPS-II necessitates a collaboration of research fields which are usually not in close contact: the know-how of large particle physics accelerator projects, optical expertise from gravitational wave interferometers and background-free single photon counting similar to the requirements in quantum optics and communication technology. Based on the experience with ALPS-I we are confident to realize ALPS-II with data taking completed in 2017 given the resources and the time schedule presented in this TDR.

ALPS-II could be implemented at DESY in a very cost effective manner by re-using available spare HERA dipole magnets and the HERA infrastructure. For investments and operation about 2 MEuro are required in total within the next five years. To significantly go beyond the ALPSII reach, R\&D activities to tackle technological challenges have been identified: Examples are a further increase of the laser power in the production cavity to several MW and operating with green light allowing for much longer installations due to a smaller laser beam divergence. Corresponding research has started already. Future ALPS-II-like experiments could strongly benefit from magnet development ongoing for a possible energy upgrade of LHC. However, such large and probably costly installations are far from being ready for decision. Besides the technology issues one has to await new results from astrophysics, laboratory WISP searches and theory.

To conclude: Although challenges remain, the rewards of exploring the low-energy frontier of particle physics with ALPS-II at DESY could be enormous, yielding fundamental insights into long-standing astro and particle physics puzzles. 


\section{Acknowledgments}

The ALPS collaboration gratefully acknowledges support by the LEXI Hamburg and DFG-SFB 676. Also, the ALPS collaboration cordially acknowledges the advice and active support of a number of people who have contributed to the $R \& D$ of the proposed experiment or the preparation of this TDR.

For advice and helpful discussions in theory and phenomenology we thank P. Arias (Universidad Católica, Santiago, Chile), C. Burrage (University of Nottingham), M. Cicoli (ICTP Trieste), M. Goodsell (CPhT, Paris), J. Jaeckel ( University of Heidelberg), F. Karbstein (Helmholtz Institute Jena) and J. Redondo (MPI Munich ASC Munich).

For advice or active support on optics, opto-mechanics and control we thank M. Frede (neoLASE GmbH), T. Meier, P. Schauzu and A. Weidner (AEI).

Regarding the development of the detection system we thank F. Borges, K. Ehret, O. Hellmig, M. Kowalski, H. Maser, A. Zuber (DESY) and G. Wiedemann (University of Hamburg), as well as our Italian TES collaborators G. Di Giuseppe, M. Karuza, M. Lucamarini, R. Natali, D. Vitali and his local crew from the University of Camerino and G. Cantatore with his team from INFN and University of Trieste. We also thank H. Barthelmess (Magnicon GmbH), U. Öchsner (Schäfter + Kirchhoff GmbH), K. Phelan, and D. Wernicke (ENTROPY GmbH). For introducing us to the TES community and much very valuable support we thank J. Beyer, I. Novikov, M. Schmidt and the local crew from PTB Berlin as well as D. Fukuda (AIST Japan), S. W. Nam (NIST USA), L. Lolli (INRIM Torino), M. Guistina, A. Mech, R. Ursin (University of Vienna) and A. J. Miller (Albion College USA).

For advice and support regarding the development of the magnet, vacuum and cryogenics systems we thank the DESY staff, in particular C. Albrecht, S. Baark, R. Bandelmann, W. Benecke, M. Berg, M. Böhnert, H. Brück, F. Czempik, H.J. Eckoldt, U. Eggerts, K. Escherich, J. Eschke,P.D. Gall, C. Hagedorn, B. Hager, U. Hahn, K. Harries, S. Holm, D. Hoppe, K. Jensch, A. Jung, S. Karstensen, L. Klein, A. Kock, M. Körfer, T. Kurps, U. Laatzen, N. Lass, A. Leuschner, H. Lewin, H.P. Lohrmann, K. Ludwig, G. Meyer, M. Noack, F. Obier, U. Packheiser, C. Peitzmann, O. Peters, B. Petersen, K. Petersen, J. Prenting, B. Racky, U. Reinhold, H. Remde, S. RettigLabusga, J. Schaffran, B. Schmidt, T. Schnautz, C. Schulz, M. Schwalger, C. Schwalm, D. Sellman, M. Staack, L. Steffen, M. Stolper, J. Teichmeier, N. Tesch, K. Tiedtke, P. Toedten, W. von Schröder, H.P. Wedekind, G. Weichert, G. Wolgast and J. Zajac.

From DESY we thank also W. Bialowons, C. Bührig, B. Conrad, U. Djunda, M. Ebert, B.D. Elie, M. Köpke, B. Lange, A. Matheisen, N. Meyners, H. Ruge, A. Schleiermacher, M. Schlösser, B. Schmidt, S. Schrader, B. Sparr, F.R. Ullrich, S. Warratz and O. Wrobel for their support and advice concerning the cleanroom construction.

For uncountable and fascinating discussions of WISP physics as well as for the fun of organizing workshops, we thank A. Afanasev (Jefferson Laboratory and George Washington University), V. Anastassopoulos (University of Patras), O. K. Baker (Yale University), L. Baudis (Zurich University), J. Jaeckel (University of Heidelberg), J. Redondo (MPI Munich), M. Schumann (Uni- 
versity of Bern) D. Tanner (University of Florida), W. Wester (FNAL), K. Zioutas (University of Patras and CERN) as well as all the attendees and contributors to the PATRAS worshop series.

We are grateful to PIER at Hamburg for supporting the WISPy lecture day and the ALPS seminar series.

Last but not least: For a careful proofreading of the manuscript, we thank U. Schneekloth as well as P. Hendrikman Verstegen. Any remaining mistakes are to be blamed to the authors of this TDR.

\section{References}

[1] J. Jaeckel and A. Ringwald, The low-energy frontier of particle physics, Ann.Rev.Nucl.Part.Sci. 60 (2010) 405-437, arXiv:1002.0329].

[2] A. Ringwald, Exploring the Role of Axions and Other WISPs in the Dark Universe, Phys.Dark Univ. 1 (2012) 116-135, arXiv:1210.5081].

[3] M. Ahlers, J. Jaeckel, and A. Ringwald, The discovery potential of laser polarization experiments, Phys.Rev. D79 (2009) 075017, arXiv:0812.3150.

[4] A. Ringwald, Production and detection of very light bosons in the HERA tunnel, Phys.Lett. B569 (2003) 51-56, hep-ph/0306106.

[5] K. Ehret, M. Frede, E.-A. Knabbe, D. Kracht, A. Lindner, et al., Production and detection of axion-like particles in a HERA dipole magnet: Letter-of-intent for the ALPS experiment, hep-ex/0702023.

[6] K. Ehret, M. Frede, S. Ghazaryan, M. Hildebrandt, E.-A. Knabbe, et al., New ALPS results on hidden-sector lightweights, Phys.Lett. B689 (2010) 149-155, arXiv:1004.1313].

[7] ALPS collaboration Collaboration, K. Ehret et al., Resonant laser power build-up in ALPS: A 'Light-shining-through-walls' experiment, Nucl.Instrum.Meth. A612 (2009) 83-96, arXiv:0905.4159].

[8] J. Redondo and A. Ringwald, Light shining through walls, Contemp.Phys. 52 (2011) 211-236, arXiv:1011.3741.

[9] A. Afanasev, O. Baker, and K. McFarlane, Production and detection of very light spin-zero bosons at optical frequencies, hep-ph/0605250.

[10] A. Afanasev, O. Baker, K. Beard, G. Biallas, J. Boyce, et al., New experimental limit on optical photon coupling to neutral, scalar bosons, Phys.Rev.Lett. 101 (2008) 120401, arXiv:0806.2631].

[11] GammeV (T-969) Collaboration, A. S. Chou et al., Search for axion-like particles using a variable baseline photon regeneration technique, Phys.Rev.Lett. 100 (2008) 080402, arXiv:0710.3783.

[12] J. H. Steffen and A. Upadhye, The GammeV suite of experimental searches for axion-like particles, Mod.Phys.Lett. A24 (2009) 2053-2068, [arXiv:0908.1529].

[13] OSQAR Collaboration, P. Pugnat et al., First results from the OSQAR photon regeneration experiment: No light shining through a wall, Phys.Rev. D78 (2008) 092003, [arXiv:0712.3362]. 
[14] OSQAR Collaboration, M. Schott et al., First results of the full-scale OSQAR photon regeneration experiment, arXiv:1110.0774.

[15] C. Robilliard, R. Battesti, M. Fouche, J. Mauchain, A.-M. Sautivet, et al., No light shining through a wall, Phys.Rev.Lett. 99 (2007) 190403, [arXiv:0707.1296].

[16] M. Fouche, C. Robilliard, S. Faure, C. Rizzo, J. Mauchain, et al., Search for photon oscillations into massive particles, Phys.Rev. D78 (2008) 032013, arXiv:0808.2800.

[17] R. Cameron, G. Cantatore, A. Melissinos, G. Ruoso, Y. Semertzidis, et al., Search for nearly massless, weakly coupled particles by optical techniques, Phys.Rev. D47 (1993) 3707-3725.

[18] M. Schott, private communication, .

[19] G. Mueller, P. Sikivie, D. B. Tanner, and K. van Bibber, Resonantly-enhanced axion-photon regeneration, AIP Conf.Proc. 1274 (2010) 150-155.

[20] P. Sikivie, Experimental tests of the invisible axion, Phys.Rev.Lett. 51 (1983) 1415.

[21] F. Wilczek, Problem of strong $p$ and $t$ invariance in the presence of instantons, Phys.Rev.Lett. 40 (1978) 279-282.

[22] S. Weinberg, A new light boson?, Phys.Rev.Lett. 40 (1978) 223-226.

[23] R. Peccei and H. R. Quinn, CP conservation in the presence of instantons, Phys.Rev.Lett. 38 (1977) $1440-1443$.

[24] CAST Collaboration, S. Aune et al., CAST search for sub-eV mass solar axions with 3 He buffer gas, Phys.Rev.Lett. 107 (2011) 261302, [arXiv:1106.3919].

[25] M. Meyer, D. Horns, and M. Raue, First lower limits on the photon-axion-like particle coupling from very high energy gamma-ray observation, arXiv: 1302.1208.

[26] K. van Bibber and G. Carosi, Status of the ADMX and ADMX-HF experiments, arXiv:1304.7803.

[27] J. Hewett, H. Weerts, R. Brock, J. Butler, B. Casey, et al., Fundamental physics at the intensity frontier, arXiv:1205.2671.

[28] P. Arias, D. Cadamuro, M. Goodsell, J. Jaeckel, J. Redondo, et al., WISPy cold dark matter, JCAP 1206 (2012) 013, arXiv:1201.5902].

[29] M. Cicoli, M. Goodsell, and A. Ringwald, The type IIB string axiverse and its low-energy phenomenology, arXiv:1206.0819.

[30] G. Raffelt, Limits on a CP-violating scalar axion-nucleon interaction, Phys.Rev. D86 (2012) 015001, arXiv:1205.1776].

[31] D. Dubbers and M. G. Schmidt, The Neutron and Its Role in Cosmology and Particle Physics, Rev.Mod.Phys. 83 (2011) 1111-1171, [arXiv:1105.3694].

[32] O. Buchmueller, R. Cavanaugh, M. Citron, A. De Roeck, M. Dolan, et al., The CMSSM and NUHM1 in light of $7 \mathrm{TeV}$ LHC, $B_{s}$ to ти+ти- and XENON100 Data, arXiv:1207.7315.

[33] O. K. Baker, M. Betz, F. Caspers, J. Jaeckel, A. Lindner, et al., Prospects for searching axion-like particle dark matter with dipole, toroidal and wiggler magnets, Phys.Rev. D85 (2012) 035018, arXiv:1110.2180.

[34] I. G. Irastorza and J. A. Garcia, Direct detection of dark matter axions with directional sensitivity, JCAP 1210 (2012) 022, arXiv:1207.6129]. 
[35] D. Horns, J. Jaeckel, A. Lindner, A. Lobanov, J. Redondo, et al., Searching for WISPy Cold Dark Matter with a Dish Antenna, arXiv:1212.2970.

[36] A. Eichhorn, H. Gies, and D. Roscher, Renormalization Flow of Axion Electrodynamics, Phys.Rev. D86 (2012) 125014, [arXiv:1208.0014].

[37] J. Jaeckel, E. Masso, J. Redondo, A. Ringwald, and F. Takahashi, The need for purely laboratory-based axion-like particle searches, Phys.Rev. D75 (2007) 013004, hep-ph/0610203.

[38] D. Horns and M. Meyer, Indications for a pair-production anomaly from the propagation of VHE gamma-rays, JCAP 1202 (2012) 033, arXiv:1201.4711].

[39] A. De Angelis, M. Roncadelli, and O. Mansutti, Evidence for a new light spin-zero boson from cosmological gamma-ray propagation?, Phys.Rev. D76 (2007) 121301, arXiv:0707.4312].

[40] M. Sanchez-Conde, D. Paneque, E. Bloom, F. Prada, and A. Dominguez, Hints of the existence of axion-like-particles from the gamma-ray spectra of cosmological sources, Phys.Rev. D79 (2009) 123511, arXiv:0905.3270].

[41] D. Horns, L. Maccione, M. Meyer, A. Mirizzi, D. Montanino, et al., Hardening of TeV gamma spectrum of AGNs in galaxy clusters by conversions of photons into axion-like particles, arXiv:1207.0776.

[42] J. Isern, L. Althaus, S. Catalan, A. Corsico, E. Garcia-Berro, et al., White dwarfs as physics laboratories: the case of axions, arXiv:1204.3565.

[43] A. H. Corsico, L. G. Althaus, M. M. M. Bertolami, A. D. Romero, E. Garcia-Berro, et al., The rate of cooling of the pulsating white dwarf star G117-B15A: a new asteroseismological inference of the axion mass, arXiv:1205.6180.

[44] R. Gill and J. S. Heyl, Constraining the photon-axion coupling constant with magnetic white dwarfs, Phys.Rev. D 84 (Oct., 2011) 085001, arXiv:1105.2083.

[45] D. Horns, L. Maccione, A. Mirizzi, and M. Roncadelli, Probing axionlike particles with the ultraviolet photon polarization from active galactic nuclei in radio galaxies, Phys. Rev. D $\mathbf{8 5}$ (Apr., 2012) 085021, [arXiv:1203.2184].

[46] B. Holdom, Two U(1)'s and epsilon charge shifts, Phys.Lett. B166 (1986) 196.

[47] S. Abel, M. Goodsell, J. Jaeckel, V. Khoze, and A. Ringwald, Kinetic mixing of the photon with hidden U(1)s in string phenomenology, JHEP 0807 (2008) 124, [arXiv: 0803.1449 ].

[48] M. Cicoli, M. Goodsell, J. Jaeckel, and A. Ringwald, Testing string vacua in the lab: From a hidden CMB to dark forces in flux compactifications, JHEP 1107 (2011) 114, [arXiv:1103.3705].

[49] W. Heisenberg and H. Euler, Consequences of Dirac's theory of positrons, Z.Phys. 98 (1936) 714-732, physics/0605038.

[50] M. Goodsell, J. Jaeckel, J. Redondo, and A. Ringwald, Naturally light hidden photons in LARGE volume string compactifications, JHEP 0911 (2009) 027, [arXiv: 0909.0515].

[51] A. E. Nelson and J. Scholtz, Dark light, dark matter and the misalignment mechanism, Phys.Rev. D84 (2011) 103501, [arXiv:1105.2812].

[52] H. Gies, J. Jaeckel, and A. Ringwald, Polarized light propagating in a magnetic field as a probe of millicharged fermions, Phys.Rev.Lett. 97 (2006) 140402, [hep-ph/0607118]. 
[53] S. A. Abel, J. Jaeckel, V. V. Khoze, and A. Ringwald, Illuminating the hidden sector of string theory by shining light through a magnetic field, Phys.Lett. B666 (2008) 66-70, [hep-ph/0608248].

[54] C. de Rham, Galileons in the sky, arXiv:1204.5492.

[55] P. Brax, C. Burrage, and A.-C. Davis, Shining Light on Modifications of Gravity, JCAP 1210 (2012) 016, arXiv:1206.1809].

[56] G. Raffelt and L. Stodolsky, Mixing of the photon with low mass particles, Phys.Rev. D37 (1988) 1237.

[57] P. Arias, J. Jaeckel, J. Redondo, and A. Ringwald, Optimizing light-shining-through-a-wall experiments for axion and other WISP searches, Phys.Rev. D82 (2010) 115018, arXiv:1009.4875].

[58] R. Battesti, M. Fouche, C. Detlefs, T. Roth, P. Berceau, et al., A photon regeneration experiment for axionlike particle search using X-rays, Phys.Rev.Lett. 105 (2010) 250405, arXiv:1008.2672].

[59] T. Inada, T. Namba, S. Asai, T. Kobayashi, Y. Tanaka, et al., Results of a Search for Paraphotons with Intense X-ray Beams at SPring-8, arXiv:1301.6557.

[60] M. Betz and F. Caspers, A microwave paraphoton and axion detection experiment with $300 \mathrm{~dB}$ electromagnetic shielding at $3 \mathrm{GHz}$, Conf.Proc. C1205201 (2012) 3320-3322, arXiv:1207.3275.

[61] J. Jaeckel and A. Ringwald, A cavity experiment to search for hidden sector photons, Phys.Lett. B659 (2008) 509-514, arXiv:0707.2063].

[62] M. Ahlers, H. Gies, J. Jaeckel, J. Redondo, and A. Ringwald, Light from the hidden sector, Phys.Rev. D76 (2007) 115005, arXiv:0706.2836.

[63] M. Ahlers, H. Gies, J. Jaeckel, J. Redondo, and A. Ringwald, Laser experiments explore the hidden sector, Phys.Rev. D77 (2008) 095001, arXiv:0711.4991].

[64] J. Jaeckel, J. Redondo, and A. Ringwald, Signatures of a hidden cosmic microwave background, Phys.Rev.Lett. 101 (2008) 131801, arXiv:0804.4157].

[65] WMAP Collaboration, E. Komatsu et al., Seven-year Wilkinson Microwave Anisotropy Probe (WMAP) observations: Cosmological interpretation, Astrophys.J.Suppl. 192 (2011) 18, arXiv:1001.4538.

[66] J. Dunkley, R. Hlozek, J. Sievers, V. Acquaviva, P. Ade, et al., The atacama cosmology telescope: cosmological parameters from the 2008 power spectra, Astrophys.J. 739 (2011) 52, [arXiv:1009.0866].

[67] Planck Collaboration Collaboration, P. Ade et al., Planck 2013 results. XVI. Cosmological parameters, arXiv:1303.5076.

[68] H. An, M. Pospelov, and J. Pradler, New stellar constraints on dark photons, arXiv:1302.3884.

[69] J. Redondo and G. Raffelt, Solar constraints on hidden photons re-visited, arXiv:1305.2920.

[70] H. An, M. Pospelov, and J. Pradler, Dark Matter Detectors as Dark Photon Helioscopes, arXiv:1304.3461.

[71] J. Jaeckel and S. Roy, Spectroscopy as a test of Coulomb's law: A probe of the hidden sector, Phys.Rev. D82 (2010) 125020, arXiv:1008.3536. 
[72] A. Mirizzi, J. Redondo, and G. Sigl, Microwave background constraints on mixing of photons with hidden photons, JCAP 0903 (2009) 026, [arXiv:0901.0014].

[73] J. G. Hartnett, J. Jaeckel, R. G. Povey, and M. E. Tobar, Resonant Regeneration in the Sub-Quantum Regime: A Demonstration of fractional quantum interference, Phys.Lett. B698 (2011) 346-352, arXiv:1101.4089.

[74] Y. e. a. Fukuda, PRODUCTION AND DETECTION OF AXIONS BY USING OPTICAL RESONATORS, Prog. Crystal Growth and Charact 33 (1996) 363-366.

[75] T. Mizumoto, R. Ohta, T. Horie, J. Suzuki, Y. Inoue, et al., Experimental search for solar hidden photons in the eV energy range using kinetic mixing with photons, arXiv: 1302.1000.

[76] M. Schwarz, A. Lindner, J. Redondo, A. Ringwald, and G. Wiedemann, Solar hidden photon search, arXiv:1111.5797.

[77] C. Burrage, J. Jaeckel, J. Redondo, and A. Ringwald, Late time CMB anisotropies constrain mini-charged particles, JCAP 0911 (2009) 002, arXiv:0909.0649].

[78] B. Dobrich, H. Gies, N. Neitz, and F. Karbstein, Magnetically amplified tunneling of the 3rd kind as a probe of minicharged particles, Phys.Rev.Lett. 109 (2012) 131802, arXiv:1203.2533.

[79] B. Dobrich, H. Gies, N. Neitz, and F. Karbstein, Magnetically amplified light-shining-through-walls via virtual minicharged particles, Phys.Rev. D87 (2013) 025022, [arXiv:1203.4986].

[80] M. Ahlers, H. Gies, J. Jaeckel, and A. Ringwald, On the particle interpretation of the PVLAS data: Neutral versus charged particles, Phys.Rev. D75 (2007) 035011, [hep-ph/0612098].

[81] F. Karbstein, L. Roessler, B. Dobrich, and H. Gies, Optical probes of the quantum vacuum: The photon polarization tensor in external fields, Int. J. Mod. Phys. Conf. Ser. 14 (2011) 403, arXiv:1111.5984.

[82] A. Melchiorri, A. Polosa, and A. Strumia, New bounds on millicharged particles from cosmology, Phys.Lett. B650 (2007) 416-420, [hep-ph/0703144].

[83] B. Dobrich, BSM photon interaction for ALPS-II and beyond, arXiv:1212.2770.

[84] F. Hoogeveen and T. Ziegenhagen, Production and detection of light bosons using optical resonators, Nucl.Phys. B358 (1991) 3-26.

[85] P. Sikivie, D. Tanner, and K. van Bibber, Resonantly enhanced axion-photon regeneration, Phys.Rev.Lett. 98 (2007) 172002, hep-ph/0701198].

[86] D. G. Blair, Advanced gravitational wave detectors / edited by David G. Blair ... [et al.]. Cambridge University Press, Cambridge, England, 2012.

[87] E. D. Black, An introduction to Pound-Drever-Hall laser frequency stabilization, American Journal of Physics 69 (Jan., 2001) 79-87.

[88] E. Morrison, B. J. Meers, D. I. Robertson, and H. Ward, Automatic alignment of optical interferometers, Appl. Opt. 33 (Aug, 1994) 5041-5049.

[89] K. Irwin and G. Hilton, Transition-Edge Sensors, in Cryogenic particle detection (C. Enss, ed.). Springer-Verlag, 2005.

[90] A. Miller, S. Nam, J. Martinis, and A. Sergienko, Demonstration of a low-noise near-infrared photon counter with multiphoton discrimination, APPLIED PHYSICS LETTERS 83 (JUL 28, 2003) 791-793. 
[91] A. E. Lita, A. J. Miller, and S. W. Nam, Counting near-infrared single-photons with $95 \%$ efficiency, OPTICS EXPRESS 16 (MAR 3, 2008) 3032-3040.

[92] G. Angloher, M. Bauer, I. Bavykina, A. Bento, A. Brown, C. Bucci, C. Ciemniak, C. Coppi, G. Deuter, F. von Feilitzsch, D. Hauff, S. Henry, P. Huff, J. Imber, S. Ingleby, C. Isaila, J. Jochum, M. Kiefer, M. Kimmerle, H. Kraus, J.-C. Lanfranchi, R. F. Lang, B. Majorovits, M. Malek, R. McGowan, V. B. Mikhailik, E. Pantic, F. Petricca, S. Pfister, W. Potzel, F. Pröbst, W. Rau, S. Roth, K. Rottler, C. Sailer, K. Schäffner, J. Schmaler, S. Scholl, W. Seidel, L. Stodolsky, A. J. B. Tolhurst, I. Usherov, and W. Westphal, Commissioning run of the CRESST-II dark matter search, Astroparticle Physics 31 (May, 2009) 270-276, [arXiv:0809.1829].

[93] P. De Korte, H. Hoevers, J. Den Herder, J. Bleeker, W. Bergmann Tiest, M. Bruijn, M. Ridder, R. Wiegerink, J. Kaastra, J. Van der Kuur, and W. Mels, Tes x-ray calorimeter-array for imaging spectroscopy, Proceedings of SPIE (2002) 779-789.

[94] A. E. Lita, B. Calkins, L. A. Pellouchoud, A. J. Miller, and S. Nam, Superconducting transition-edge sensors optimized for high-efficiency photon-number resolving detectors, in Society of photo-optical instrumentation engineers (SPIE) conference series, vol. 7681 of Society of Photo-Optical Instrumentation Engineers (SPIE) Conference Series, Apr., 2010.

[95] D. Fukuda, G. Fujii, T. Numata, K. Amemiya, A. Yoshizawa, H. Tsuchida, H. Fujino, H. Ishii, T. Itatani, S. Inoue, and T. Zama, Titanium-based transition-edge photon number resolving detector with $98 \%$ detection efficiency with index-matched small-gap fiber coupling, Opt. Express 19 (Jan, 2011) 870-875.

[96] A. E. Lita, B. Calkins, L. Pellouchoud, A. Miller, and S. Nam, Superconducting transition-edge sensors optimized for high-efficiency photon-number resolving detectors, in Proc. SPIE 7681, 2010.

[97] A. J. Miller, A. E. Lita, B. Calkins, I. Vayshenker, S. M. Gruber, and S. W. Nam, Compact cryogenic self-aligning fiber-to-detector coupling with losses below one percent, Opt. Express 19 (May, 2011) 9102-9110.

[98] E. R. Abraham and E. A. Cornell, Teflon feedthrough for coupling optical fibers into ultrahigh vacuum systems, Appl. Opt. 37 (Apr, 1998) 1762-1763.

[99] Entropy GmbH, Company for ADR cryostats, 2012. http://www.entropy-cryogenics.com.

[100] P. Instruments, Pixis: 1024, 2011. http://www.princetoninstruments.com/products/imcam/pixis/dsheet.aspx.

[101] A. Miller, A. E. Lita, D. Rosenberg, S. Gruber, and S. Nam, Superconducting photon number resolving detectors: Performance and promise, in

Proceedings of the 8th international conference on quantum communication, measurement and computing (J. Hirota, H. Shapiro, and M. Sasaki, eds.), pp. 445-450, NICT Press, 2007.

[102] P. Sikivie, Detection rates for 'invisible' axion searches, Phys.Rev. D32 (1985) 2988.

[103] T. Meier, High-power CW green lasers for optical metrology and their joint benefit in particle physics experiments. PhD thesis, Albert Einstein Institute Hannover, 2011.

[104] C. Wyss, LHC arc dipole status report, CERN-LHC-PROJECT-REPORT-288 (1999) 149-153.

[105] H. Edwards, The Tevatron energy doubler: A superconducting accelerator, Ann.Rev.Nucl.Part.Sci. 35 (1985) 605-660.

[106] G. Mueller, P. Sikivie, D. Tanner, and K. van Bibber, Detailed design of a resonantly-enhanced axion-photon regeneration experiment, Phys.Rev. D80 (2009) 072004, [arXiv:0 907.5387 ]. 
[107] W. Wester, "Reapr resonantly enhanced axion photon regeneration." Presentation at workshop 'Vistas in Axion Physics', Seattle, April, 2012.

[108] P. Mazur, "Concepts and challenges of long magnet strings." Presentation at workshop 'Vistas in Axion Physics', Seattle, April, 2012.

[109] H. Kaiser, Design of superconducting dipole for HERA, DESY HERA-1986-14, C86/08/07.

[110] PVLAS Collaboration, E. Zavattini et al., New PVLAS results and limits on magnetically induced optical rotation and ellipticity in vacuum, Phys.Rev. D77 (2008) 032006, [arXiv:0706.3419].

[111] J. Eckoldt. DESY group MKK. private communication.

[112] K. Mess, P. Schmueser, and S. Wolff, Superconducting accelerator magnets. World Scientific, Singapore, 1996.

[113] R. Niessen, Over voltage protection through potential equalization line in superconducting magnets, DESY-HERA-91-05 (1991).

[114] K. Van Bibber, N. Dagdeviren, S. Koonin, A. Kerman, and H. Nelson, Proposed experiment to produce and detect light pseudoscalars, Phys.Rev.Lett. 59 (1987) 759-762.

[115] G. Horlitz, A central refrigeration system for the superconducting HERA proton magnet ring, DESY-HERA-84-02 (1985).

[116] M. Clausen, C. Gerke, G. Horlitz, H. Lierl, K. Nowakowski, et al., Performance test of the HERA $3 x$ 6500-W helium refrigeration plant, DESY-HERA-87-18 (1987).

[117] W. Eschricht, G. Horlitz, U. Knopf, R. Lange, H. Lierl, et al., Cryogenic test of helium transfer line modules for HERA, DESY-HERA-88-11, C88/07/12 (1988).

[118] M. Bohnert, D. Brauer, R. Hensler, D. Hoppe, D. Hubert, et al., The Cold bore beam pipe vacuum system of the HERA proton storage ring, DESY-HERA-92-02 (1992).

[119] M. Bohnert, D. Brauer, S. Chermenin, H. Doruch, D. Hoppe, et al., The insulating vacuum systems of the HERA proton storage ring, DESY-HERA-91-22 (1991).

[120] E. Wallen, Experimental test of the propagation of a He pressure front in a long, cryogenically cooled tube, JOURNAL OF VACUUM SCIENCE \& TECHNOLOGY A-VACUUM SURFACES AND FILMS 15 (NOV-DEC, 1997) 2949-2958.

[121] G. Ruoso, R. Cameron, G. Cantatore, A. Melissinos, Y. Semertzidis, et al., Limits on light scalar and pseudoscalar particles from a photon regeneration experiment, Zeit.Phys. C56 (1992) 505-508.

[122] AlazarTech, "Data sheet of ats9462."

http://www.alazartech.com/products/ATS9626_v_1_1.pdf, 2012.

[123] S. Ritt, R. Dinapoli, and U. Hartmann, Application of the DRS chip for fast waveform digitizing, Nucl.Instrum.Meth. A623 (2010) 486-488.

[124] xmlrpc homepage, "Xmlrpc.com." http://xmlrpc.scripting.com/, 2012.

[125] P. und Maschinen-Automation, "Ci45 data sheet."

http://www.pma-online.de/de/pdf/db_ci45_d_9498-737-48333.pdf, 2012.

[126] N. Tesch. DESY radiation safety group D3. private communication.

[127] I. Borchardt, D. Brauer, S. Chermenin, D. Degele, K. Escherich, et al., Installation of the superconducting magnets in the HERA tunnel, Conf.Proc. C910506 (1991) 857-859. 
[128] I. Borchardt and P. Schmueser, Polarity and electric bonding of superconducting magnets and correction coils in the HERA proton ring, DESY-HERA-93-01 (1993).

[129] G. J. Feldman and R. D. Cousins, A unified approach to the classical statistical analysis of small signals, Phys.Rev. D57 (1998) 3873-3889, [physics/9711021]. 